\title{
Produção de Mésons Vetoriais em Colisões Ultra-periféricas com o Código de Monte Carlo CRISP
}

\author{
Evandro Oliveira Andrade Segundo
}

Orientador: Prof. Dr. Airton Deppman

Tese de doutorado apresentada ao Instituto de

Física da Universidade de São Paulo para a obtenção do título de Doutor em Ciências.

Banca Examinadora:

Prof. Dr. Airton Deppman - Orientador (IFUSP)

Prof. Dr. Fernando Brandt (IFUSP)

Prof. Dr. Alexandre Suaide (IFUSP)

Prof. Dr. Manuel Malheiro Oliveira (ITA)

Prof. Dr. Magno Valerio Trindade (UFRGS)

São Paulo 


\section{FICHA CATALOGRÁFICA \\ Preparada pelo Serviço de Biblioteca e Informação do Instituto de Física da Universidade de São Paulo}

Andrade Segundo, Evandro Oliveira

Produção de mésons vetoriais em colisões ultra-periféricas com o código de Monte Carlo CRISP. São Paulo, 2015.

Tese (Doutorado) - Universidade de São Paulo. Instituto de Física. Depto. de Física Experimental.

Orientador: Prof. Dr. Airton Deppman

Área de Concentração: Reações Nucleares e Espalhamento (Reações Específicas).

Unitermos: 1. Colisões de íons pesados relativísticos; 2. Reações fotonucleares; 3.Partículas (Física nuclear); 4. Método de Monte Carlo. 
A meus pais Evandro e Fátima. 



\section{Agradecimentos}

Agradeço a meus pais Evandro Andrade e Fátima Fraga, cujas palavras sábias e os exemplos sólidos tem me conduzido por um caminho de vitórias. Seu amor incondicional me trouxe até aqui.

Ao meu orientador, Prof. Dr. Airton Deppman, que tem instruído meus passos nos caminhos da ciência e me ensinado a pensar, sempre com dedicação. Verdadeiro mestre e amigo.

Ao Prof. Dr. Carlos Bertulani. Sua expertise no tema desta tese e sua co-orientação elevaram este trabalho e foram determinantes para o seu sucesso.

Ao Dr. Israel Medina. Seus avanços anteriores com o modelo CRISP lançaram as bases para esta tese.

A minha esposa Débora Pio, pelo ouvir paciente e pela compreensão. Suas palavras de apoio sempre me fortalecem.

Meu sincero agradecimento aos meus amigos, irmãos em Cristo, cujos nomes bastariam para encher esta página, que sempre fizeram da minha alegria também a deles.

Acima de tudo, agradeço ao meu Senhor Jesus, que me deu os pais, os mestres, os colegas, a esposa e os amigos, assim como me deu a benção de conhecer e estudar a obra de Suas mãos. A Ele seja a Glória!

Agradeço ainda à Fundação de Amparo à Pesquisa do Estado de São Paulo - FAPESP, que apoiou este trabalho por meio do financiamento 2012/13337-0. 

"It has long been an axiom of mine that the little things are infinitely the most important".

Sir Arthur Conan Doyle, (Sherlock

Holmes) A Case of Identity, 1892 



\section{Resumo}

Este trabalho é dedicado ao estudo da fotoprodução de mésons vetoriais em colisões ultraperiféricas. Este estudo foi realizado por meio do código de Monte Carlo CRISP, o qual recebeu atualizações e implementações a fim de permitir o cálculo das colisões. Foi elaborado um modelo para a geração do espectro de energia dos fótons equivalentes que deve interagir com o alvo, bem como foram introduzidos novos canais de foto-absorção para a fotoprodução de múltiplos píons, os quais são essenciais à reprodução dos fenômenos nucleares em altas energias. Ao todo 84 canais foram introduzidos. Foi estudada, em particular, a produção do méson $\mathrm{J} / \psi$ em colisões $\mathrm{Pb}-\mathrm{Pb}$ a $\sqrt{s_{N N}}=2.76 \mathrm{TeV}$ e em colisões Au-Au a $\sqrt{s_{N N}}=200 \mathrm{GeV}$.

Palavras-chave: Monte Carlo, colisão ultra-periférica, mésons vetoriais. 



\section{Abstract}

This work is dedicated to the study of the photoproduction of vector mesons in ultraperipheral collisions. This study was accomplished by means of the Monte Carlo code CRISP which gained updates and implementations in order to allow the calculation of the collisions. A model for the generation of the spectrum of equivalent photons that interact with the target was designed. Also, new photo-absorption channels for the multipion photoproduction were introduced in the code. They are essential to the description of the nuclear environment at high energies. Altoghether, 84 channels were implemented. In particular, the production of the $J / \psi$ vector meson in $\mathrm{Pb}-\mathrm{Pb}$ collision at $\sqrt{s_{N N}}=2.76 \mathrm{TeV}$ and in $\mathrm{Au}-\mathrm{Au}$ collisions at $\sqrt{s_{N N}}=200$ $\mathrm{GeV}$ was studied.

Keywords: Monte Carlo, ultra-peripheral collisions, vector mesons. 



\section{Sumário}

\section{Lista de Figuras}

1 Introdução $\quad$ p. 19

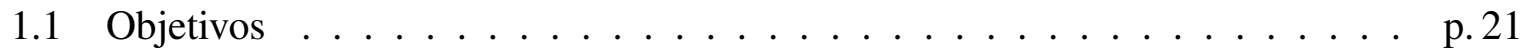

2 Fundamentação teórica $\quad$ p. 23

2.1 Colisões Ultra-periféricas . . . . . . . . . . . . . . . p. 23

2.1.1 Método de Weiszäcker-Williams . . . . . . . . . . . . p. 23

2.1.2 Probabilidade de Sobrevivência . . . . . . . . . . p. 28

2.1.3 Seção de Choque em Colisões Ultra-periféricas . . . . . . . . . . . . p. 29

2.2 Teoria de Regge . . . . . . . . . . . . . . . . . p. . 32

2.2.1 Trajetórias de Regge e Amplitude de Espalhamento . . . . . . . . . p. 33

2.3 Foto-produção de Mésons Vetoriais . . . . . . . . . . . . p. 36

2.3.1 Interações de Estado Final . . . . . . . . . . . . . . . . p. 39

2.4 Produção de Mésons numa Colisão Ultra-periférica $\ldots \ldots$. . . . . . . . p. p. 45

2.4.1 Produção Coerente de Mésons Vetoriais . . . . . . . . . . . . p. 48

2.4.2 Produção Incoerente de Mésons Vetoriais . . . . . . . . . . . . p. 50

2.5 Fotoprodução de Múltiplos Píons - Foto-absorção . . . . . . . . . . . p. 51 
3 Ferramentas Computacionais $\quad$ p. 53

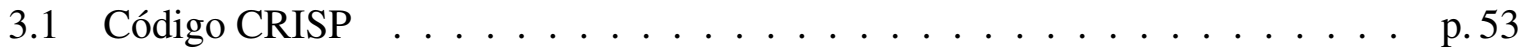

3.1.1 Modelo de Cascata Intranuclear . . . . . . . . . . . . p. 55

3.2 Plataforma ROOT . . . . . . . . . . . . . . p. 61

3.3 Outras Ferramentas $\ldots \ldots \ldots \ldots$ p. 62

4 Resultados e Discussão $\quad$ p. 63

4.1 Fotoprodução de Múltiplos Píons - Foto-absorção . . . . . . . . . . . . . p. 63

4.2 Fotoprodução do méson $\mathrm{J} / \psi \ldots \ldots \ldots \ldots \ldots \ldots$. . . . . . . . . . . . . . .

5 Conclusões $\quad$ p. 87

$\begin{array}{lr}\text { Referências Bibliográficas } & \text { p. } 89\end{array}$ 


\section{Lista de Figuras}

2.1 Partícula carregada passando por um sistema $S$ e o pulso equivalente de radi-

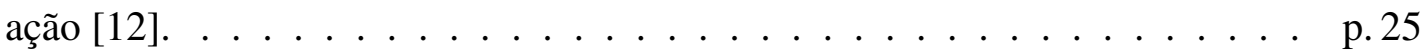

2.2 Espectro de frequência dos dois pulsos de radiação [12]. . . . . . . . . . . . p. 26

2.3 Influência do parâmetro de impacto $b$ sobre o espectro de energia dos fótons virtuais. Reproduzido da Referência [13]. . . . . . . . . . . . . . . . p. 27

2.4 Probabilidade de sobrevivência de íons em uma colisão $\mathrm{Pb}-\mathrm{Pb} . \quad \ldots \ldots$. . . . . p. 29

2.5 Fluxo de fótons virtuais em uma colisão $\mathrm{PbPb}$ a $\sqrt{s_{N N}}=2.76 \mathrm{TeV} \ldots \ldots$. . . p. 30

2.6 Exemplos de traetórias de Regge. Extraído da Referência [18]. . . . . . . . . p. 34

2.7 Esquema para uma colisão relativística. . . . . . . . . . . . . . . p. 34

2.8 Esquemas para uma colisão relativística genérica representando os canais s, $\mathrm{t}$

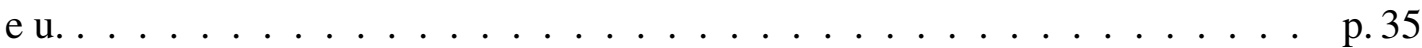

2.9 Seção de choque elástica de fotoprodução de mésons vetoriais em interação com proton. $W=\sqrt{s_{\gamma N}}$. Extraído de [6] . . . . . . . . . . . p. 38

2.10 Diagramas de Feynman. a) foto-produção de méson vetorial. b) a d) FSI para o méson $\omega$. Extraído de $[6] . \ldots \ldots \ldots$. . . . . . . . . . . . . . . . . . .

2.11 Diagramas de dissociação do méson $J / \psi[6] . \ldots \ldots \ldots$. . . . . . . p. 42

2.12 Distribuição de $p_{T}$ para di-múons (à esquerda) e di-elétrons (à direita) para a colisão ultra-periférica $\mathrm{PbPb}$ a $\sqrt{s_{N N}}=2.76 \mathrm{TeV}$ dentro do intervalo de rapidez $-0.9<y<0.9$. Dados ajustados a partir de 6 distribuições calculadas por Monte Carlo. Extraído de [8]. . . . . . . . . . . . . . . . p.47 
3.1 Visualização do efeito de sombreamento devido à hadronização do fóton energético. Os pontos em cores foram inteiramente calculados com o CRISP. O circulo vazio representa a seção de choque experimental de fotoprodução do méson $\rho$. Extraído de $[6] \ldots \ldots \ldots \ldots \ldots \ldots$ p. . . . . . . . . .

4.1 Seções de choque de fotoprodução de múltiplos píons (Parte 1). Dados experimentais obtidos de $[39] \ldots \ldots \ldots \ldots \ldots$ p. 63

4.2 Seções de choque de fotoprodução de múltiplos píons (Parte 2). Dados experimentais obtidos de $[39] \ldots \ldots \ldots \ldots$. . . . . . . . . . . . . . . 64

4.3 Seções de choque de fotoprodução de múltiplos píons (Parte 3). Dados experimentais obtidos de $[39] \ldots \ldots \ldots \ldots$. . . . . . . . . . . . . . . 65

4.4 Exemplo do procedimento de subtração de uma contribuição por méson $\omega$ na foto-produção de píons na reação $\gamma p \longrightarrow p \pi^{+} \pi^{-} \pi^{0}$. Dados experimentais [39].

4.5 Seções de choque de fotoprodução de mútiplos píons. Dados experimentais [39]. Na Figura (b) os dados são de [64] . . . . . . . . . . . . . . . . . . . . p.74

4.6 Seção de choque de produção do méson $\mathrm{J} / \psi$ no próton em função da energia no centro de massa do par $\gamma p$. Dados experimentais obtidos pela Colaboração ALICE por meio de medidas em colisão ultraperiférica p- $\mathrm{Pb}$ [65].

4.7 Seção de choque incoerente de fotoprodução do méson $\mathrm{J} / \psi$ em função da rapidez mostrando a contribuição de ambos os núcleos de $\mathrm{Pb}$ a $\sqrt{s_{N N}}=2.76$ $\mathrm{TeV}$.

4.8 Seção de choque incoerente de fotoprodução de J/ $\psi$. Comparação com diferentes modelos

4.9 a) Distribuição de momento transversal do méson $\mathrm{J} / \psi$ obtida pelo modelo CRISP em comparação com o modelo STARLIGHT. b) Seção de choque incoerente de fotoprodução do méson $\mathrm{J} / \psi \ldots \ldots \ldots$. . . . . . . . . . . 
4.10 Seção de choque incoerente de fotoprodução de $\mathrm{J} / \psi$ em diferentes cenários. p. 81

4.11 Distribuição da posição de criação do méson $\mathrm{J} / \psi$ conforme ele seja posteriormente emitido ou absorvido pelo núcleo. . . . . . . . . . . . . p.82

4.12 Probabilidade de sobrevivência obtida a partir de um ajuste extremo de parâ-

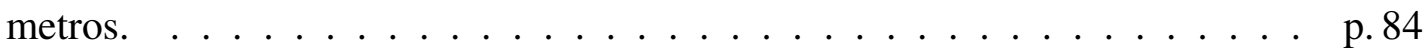

4.13 Seção de choque total de fotoprodução de $\mathrm{J} / \psi$ para colisão AuAu a $\sqrt{s_{N N}}=$ 200 GeV. Comparação com os modelos STARLIGHT, Strikman et al e GonçalvesMachado, todos extraídos de [68] assim como o ponto experimental. As linhas $(-\cdot)$ delimitam a incerteza estatística no cálculo. . . . . . . . . . . p.85 



\section{$1 \quad$ Introdução}

Foto-reações sempre foram, e é provável que continuem sendo, uma das ferramentas mais importantes em Física Nuclear.

Reações induzidas por fótons sempre atrairam atenção, tanto em núcleos leves como pesados [1, 2]. Fótons já se mostraram mais adequados ao estudo de ressonânicas Delta [2] do que píons, por exemplo, devido à possibilidade de interagirem com mais nucleons no interior do núcleo. A fotofissão já tem sido utilizada há algum tempo no estudo do mecanismo de fotoabsorção, de modo que a própria seção de choque total de fotoabsorção, de difícil medição em particular para núcleos de alto Z, tem sido substituída pela seção de choque total de fotofissão, já que a fissão é um canal importante no estudo de núcleos pesados a energias maiores [3]. Ressonâncias gigantes, um fenômeno de baixas energias, ainda é pouco compreendido e continua sendo estudado. De fato, ressonâncias gigantes podem ser estudadas em colisores de íons pesados por meio de excitação eletromagnética, método que tem se mostrado útil e uma alternativa ao uso de píons [4]. Além disso, em colisores de núcleos pesados relativísticos, ressonâncias gigantes, em grande parte de dipolo simples, são um canal recorrente de perda de feixe [5].

O foco da pesquisa com foto-reações também é compartilhado com o estudo de canais de fotoabsorção que se abrem a energias mais altas, como canais de produção de mésons vetoriais, que podem ser observados desde $1 \mathrm{GeV}$ na energia do centro de massa do par $\gamma p$ [6].

De fato, foto-reações são uma ferramenta chave na investigação da matéria nuclear, e os aceleradores modernos elevaram o poder da investigação científica a um novo patamar ao criarem condições experimentais relativísticas e mais recentemente ultra-relativísticas. No Relativistic Heavy Ion Collider (RHIC), alcança-se, em colisões Au-Au, a energia de $\sqrt{s_{N N}}=200 \mathrm{GeV}$, 
onde $\sqrt{s_{N N}}$ é a energia no centro de massa de um par de nucleons, e $\sqrt{s}=500 \mathrm{GeV}$ em colisões p-p. No Large Hadron Collider (LHC), chega-se a $\sqrt{s_{N N}}=2.76 \mathrm{TeV}$ em colisões $\mathrm{Pb}-\mathrm{Pb}$ e mais recentemente a $\sqrt{s}=14 \mathrm{TeV}$ em colisões p-p. Nessas condições, o campo eletromagnético de cada íon sofre forte contração de Lorentz na direção do feixe a ponto de se aproximar de um intenso pulso eletromagnético. Tal campo pode ser modelado como um fluxo de fótons virtuais. Os detalhes teóricos desse modelo serão tratados no próximo capítulo. Quando os íons passam um pelo outro sem haver interação hadrônica ainda assim os campos de cada um podem ser sentidos pela matéria nuclear de cada íon e a colisão é dita periférica, ou ultra-periférica em caso de regime ultra-relativístico, com a presença de interações de caráter fotonuclear. A relevância que foto-reações passam a ter nesse tipo de ambiente experimental é alta. Sem dúvida, o monitoramento da colisão é um aspecto importante, já que como mencionado no segundo parárafo desta introdução, excitações eletromagnéticas deterioram o feixe, mas há física importante ainda por ser desvendada a respeito da matéria nuclear com o auxílio de foto-reações.

Embora os aceleradores modernos não tenham sido construídos com o fim último de estudar colisões ultra-periféricas, a vantagem tecnológica de usá-los para esse propósito não passou despercebida e o interesse por estudar fóto-reações em regimes relativísticos e ultra-relativísticos tem crescido. Durante a tomada de dados na colisão $\mathrm{Pb}-\mathrm{Pb}$ realizada no LHC em 2011, um trigger dedicado para a seleção de eventos de caráter ultra-periférico já estava em funcionamento e resultados sobre a foto-produção do méson vetorial $J / \psi$ foram recentemente publica$\operatorname{dos}[7,8,9]$.

Foto-reações a energias tão altas quanto às que são praticadas nesses regimes permitem investigar a matéria nuclear numa profundidade antes não alcançada. A distribuição nuclear de glúons ainda não é um fato elucidado e tal propriedade pode ter grande relevância na compreensão das seções de choque de fotoprodução de mésons vetoriais, por exemplo, visto que a distribuição nuclear de glúons introduz efeitos de sombreamento. Razão pela qual tais seções de choque tem sido medidas, pois servem de meio indireto de investigação. Diversos modelos tem tentado encontrar a correta distribuição de glúons que leva por sua vez ao cálculo correto das seções de choque. 
Os exemplos acima são, em parte, alguns do benefícios já colhidos com o estudo de fotoreações, e também uma mostra do que ainda se pode desvendar por meio desse novo caminho que se abriu para a Física Nuclear com o estudo de colisões ultra-periféricas em colisores em pleno funcionamento.

\subsection{Objetivos}

O objetivo deste trabalho está centrado na produção de mésons vetoriais em colisões ultraperiféricas, ou UPCs (sigla em inglês para ultra-peripheral collisions), especialmente em colisões íon-1́on.

Para tanto, algumas etapas foram necessárias, e de maneira específica se objetivou:

- Inserir novos canais de fotoabsorção a altas energias, em particular foto-produção de múltiplos píons;

- Construir o modelo a partir do qual se pode obter o fluxo de fótons virtuais, também nomeados fótons equivalentes;

- Reproduzir as condições encontradas em UPC, a saber:

- Calcular a interação fotonuclear de cada íon participante;

- Incorporar ao resultado obtido o fluxo de fótons virtuais adequado à colisão, convertendo assim o processo fotonuclear simples em um processo ultra-periférico;

- Cálcular a seção de choque de foto-produção do méson J/ $\psi$ e sua distribuição de momento transversal.

Canais de foto-absorção com subsequente produção de mésons vetoriais já existem no código CRISP, embora não tivessem sido empregados até o presente momento no estudo de UPC.

A foto-produção de múltiplos píons é essencial neste estudo pelo fato de muitos canais se abrirem com o aumento da energia. Sem a inclusão deles, o meio nuclear não seria devidamente 
reproduzido, o que comprometeria, por exemplo, o cálculo de várias interações de estado final. Algumas interações de estado final com píons são responsáveis pela produção de alguns mésons vetoriais [6] e várias delas podem se seguir à fotoabsorção. 


\section{Fundamentação teórica}

\subsection{Colisões Ultra-periféricas}

Colisões ultra-periféricas são aquelas nas quais dois íons passam um pelo outro sem que haja interação hadrônica, mas havendo sim excitação eletromagnética no processo devida à interação da matéria nuclear do íon de um feixe com o campo eletromagnético de um íon presente no feixe que viaja em sentido contrário.

Encontrar o modelo que melhor descreve essa situação é um desafio teórico, mas o fato é que condições semelhantes a essas já ocorreram em estudos no passado, mais precisamente na década de 20. Naturalmente, não no ambiente de um colisor nem em regime relativístico. $\mathrm{O}$ vencedor do Prêmio Nobel de 1938, Enrico Fermi, precisava calcular a excitação e ionização de átomos por meio de partículas alfa energéticas. Fermi se deu conta de que seus cálculos seriam grandemente simplificados se o campo eletromagnético gerado pelas partículas alfa fosse substituído por um campo de fótons equivalentes incidente sobre o átomo [10, 11].

Weiszäcker e Williams, de modo independente, desenvolveram o modelo de Fermi para a inclusão do caráter relativístico e atualmente tal modelo é mais comumente conhecido como método de Weiszäcker-Williams do fóton virtual ou equivalente e é descrito em detalhes a seguir.

\subsubsection{Método de Weiszäcker-Williams}

O método do fóton virtual faz uso da similaridade que há entre o campo de uma partícula carregada em movimento relativístico e um pulso de radiação, correlacionando o efeito da co- 
lisão dessa partícula com um certo sistema com a interação da radiação, neste caso, o fóton virtual, com o tal sistema. Essa correlação é possível já que a interação entre os participantes da colisão é apenas eletromagnética.

As colisões ultra-periféricas reproduzem o cenário necessário à aplicação do método.

Em uma colisão, ficam definidos uma "partícula incidente" e um "sistema atingido". Os campos da partícula incidente são substituídos por um pulso equivalente de radiação conforme um espectro de frequência de fótons virtuais. Os efeitos da interação do fóton virtual, seja absorção ou espalhamento, podem ser calculados.

O espectro de radiação equivalente para uma partícula carregada de carga $q$, velocidade $v \simeq c$, passando por um sistema $S$ com um parâmetro de impacto $b$, pode ser obtido a partir dos campos elétrico e magnético [12],

$$
\begin{aligned}
& E_{2}(t)=q \frac{\gamma b}{\left(b^{2}+\gamma^{2} v^{2} t^{2}\right)^{3 / 2}} \\
& B_{3}(t)=\beta E_{2}(t) \\
& E_{1}(t)=-q \frac{\gamma v t}{\left(b^{2}+\gamma^{2} v^{2} t^{2}\right)^{3 / 2}}
\end{aligned}
$$

onde $\beta=v / c$ e

$$
\gamma=\left(1-v^{2} / c^{2}\right)^{-1 / 2}
$$

é o fator de contração de Lorentz.

Para $\beta \simeq 1$ os campos $E_{2}(t)$ e $B_{3}(t)$ são equivalentes ao pulso de um radiação polarizada $P_{1}$ incidente em $S$ na direção $x_{1}$, como mostrado na Figura 2.1.

De fato, não há um campo magnético que acompanhe $E_{1}(t)$ de modo a formar o pulso de radiação $P_{2}$ na direção $x_{2}$ na figura, visto que a componente $E_{1}(t)$ coincide com a direção de propagação da partícula carregada de modo que a componente $x_{1}$ de $\vec{B}=\vec{\beta} \times \vec{E}$ é nula. Porém, pode-se adicionar o campo magnético necessário para a formação do pulso $P_{2}$ sem prejuízo da física do problema se as partículas são relativísticas pois o pulso de radiação $P_{2}$ adicional na verdade tem importância reduzida no espectro final de frequências como será visto adiante. 

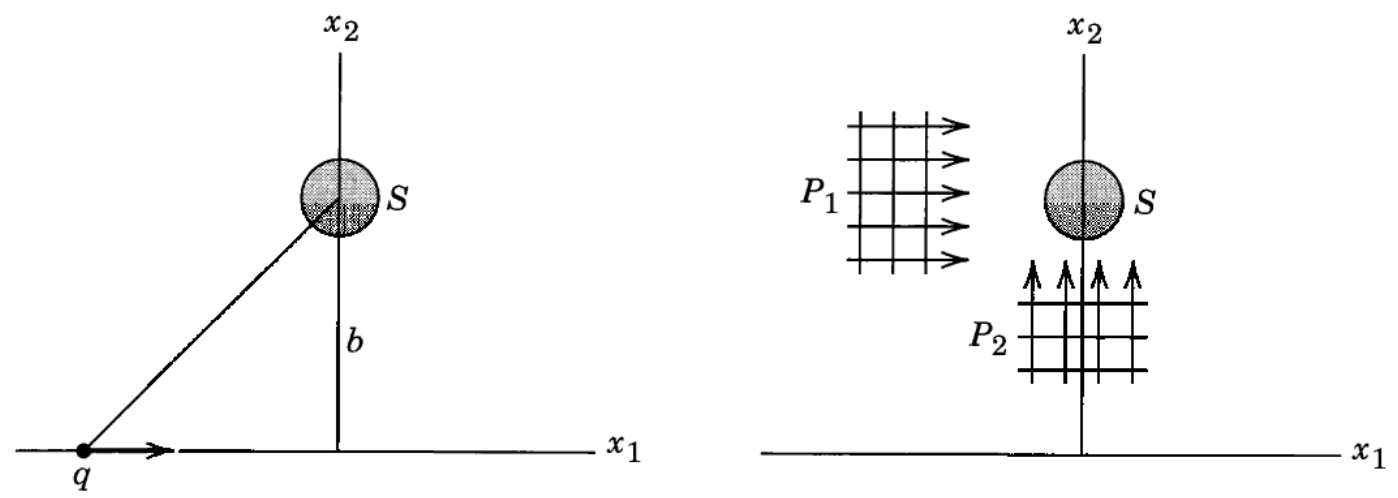

Figura 2.1: Partícula carregada passando por um sistema $S$ e o pulso equivalente de radiação [12].

O pulso $P_{1}$ possui um espectro de frequência (energia por unidade de área, por unidade de intervalo de frequência) dado por

$$
\frac{d I_{1}}{d \omega}(\omega, b)=\frac{c}{2 \pi}\left|E_{2}(\omega)\right|^{2}
$$

sendo $E_{2}(\omega)$ a transformada de Fourier de $E_{2}(t)$. Quanto ao pulso $P_{2}$, tem-se o espectro

$$
\frac{d I_{2}}{d \omega}(\omega, b)=\frac{c}{2 \pi}\left|E_{1}(\omega)\right|^{2}
$$

De modo que os dois espectros de frequência são

$$
\begin{aligned}
& \frac{d I_{1}}{d \omega}(\omega, b)=\frac{1}{\pi^{2}} \frac{q^{2}}{c}\left(\frac{c}{v}\right)^{2} \frac{1}{b^{2}}\left(\frac{\omega b}{\gamma \nu}\right)^{2} K_{1}^{2}\left(\frac{\omega b}{\gamma \nu}\right) \\
& \frac{d I_{2}}{d \omega}(\omega, b)=\frac{1}{\pi^{2}} \frac{q^{2}}{c}\left(\frac{c}{v}\right)^{2} \frac{1}{b^{2}} \frac{1}{\gamma^{2}}\left(\frac{\omega b}{\gamma \nu}\right)^{2} K_{0}^{2}\left(\frac{\omega b}{\gamma \nu}\right),
\end{aligned}
$$

onde $K_{0}$ e $K_{1}$ são as funções de Bessel modificadas de segundo tipo.

A forma do espectro de cada pulso pode ser vista na Figura 2.2, onde se nota que o fator $1 / \gamma^{2}$ na expressão para $P_{2}$ é o responsável pela sua menor importância para partículas relativísticas.

Em problemas de colisão, deve-se integrar o espectro sobre todos os parâmetros de impacto possíveis. Daí se tem a energia por unidade de intervalo de frequência presente no campo de radiação equivalente. Dada a necessidade de que a interação seja de caráter apenas eletromagnético, é extensivamente considerado na literatura que se defina um parâmetro de impacto 


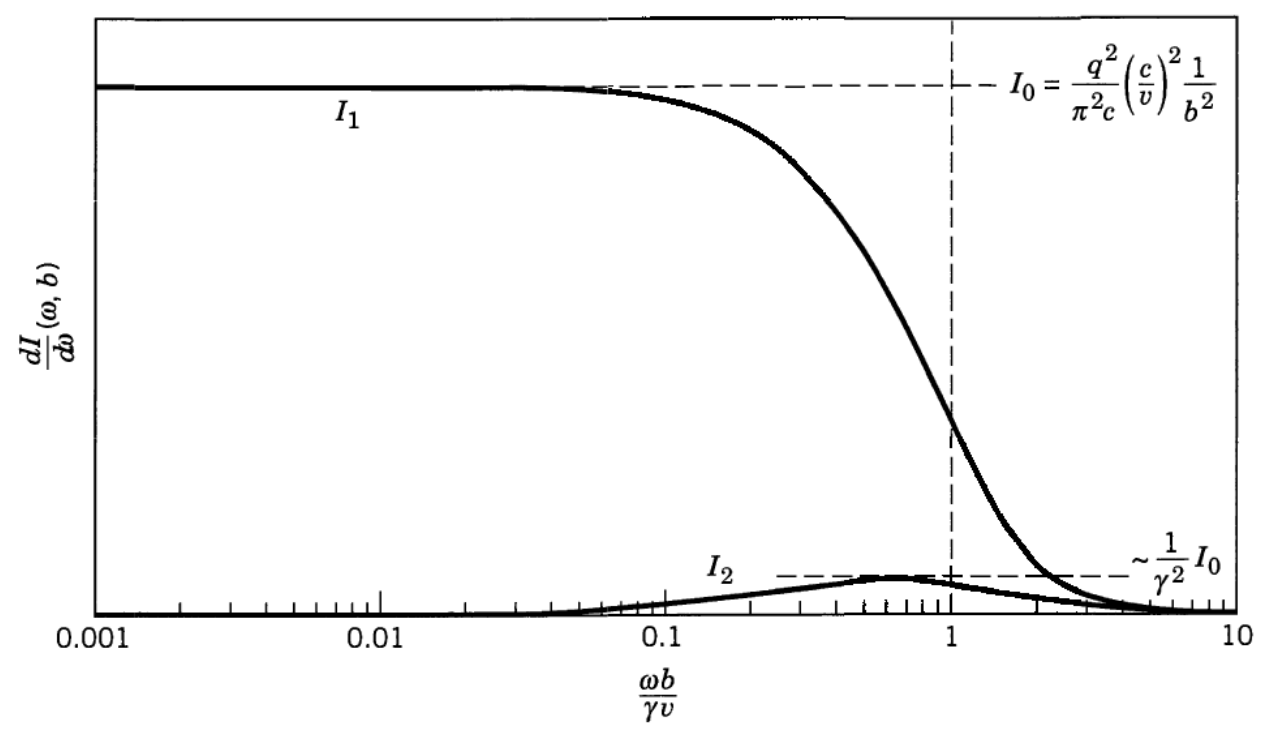

Figura 2.2: Espectro de frequência dos dois pulsos de radiação [12].

mínimo, $b_{\min }=R_{1}+R_{2}$, onde $R_{1}$ e $R_{2}$ são os raios das partículas em colisão. Dessa forma, temos

$$
\frac{d I}{d \omega}(\omega)=2 \pi \int_{b_{\min }}^{\infty}\left[\frac{d I_{1}}{d \omega}(\omega, b)+\frac{d I_{2}}{d \omega}(\omega, b)\right] b d b,
$$

cujo resultado é

$$
\frac{d I}{d \omega}(\omega)=\frac{2}{\pi^{2}} \frac{q^{2}}{c}\left(\frac{c}{v}\right)^{2}\left\{x K_{0}(x) K_{1}(x)-\frac{v^{2}}{2 c^{2}} x^{2}\left[K_{1}^{2}(x)-K_{0}^{2}(x)\right]\right\}
$$

onde

$$
x=\frac{\omega b_{\min }}{\gamma v}
$$

O espectro em número de fótons virtuais, $N(\hbar \omega)$, é obtido a partir da relação

$$
\frac{d I}{d \omega}(\omega) d \omega=\hbar \omega N(\hbar \omega) d(\hbar \omega)
$$

Para os propósitos deste trabalho é mais conveniente partir diretamente da Equação (2.5) usando a relação (2.9) e escrever o espectro de energia em número de fótons virtuais na forma

$$
n\left(E_{\gamma}, b\right)=\frac{Z_{1}^{2} \alpha}{\pi^{2}} \frac{1}{\beta^{2} b^{2}} \frac{x^{2}}{E_{\gamma}}\left[K_{1}^{2}(x)+\frac{1}{\gamma^{2}} K_{0}^{2}(x)\right]
$$


onde $E_{\gamma}=\hbar \omega, Z_{1}$ é a carga do núcleo considerado projétil, $\alpha$ é a constante de estrutura fina e reescrevemos $x=\frac{E_{\gamma} b}{\hbar \gamma \beta c}$.

Ainda é importante considerar a dependência que o espectro de energia dos fótons virtuais tem com respeito ao parâmetro de impacto $b$ da colisão. A Figura 2.3 ilustra a situação. Foi assumido o referencial do alvo, visualizado como uma esfera, enquanto o projétil aparece no típico formato panqueca devido à contração de Lorentz na direção do feixe.

$$
\begin{aligned}
& \text { Projectile nucleus: } \quad \text { Field strength } \\
& A_{1}, Z_{1}
\end{aligned}
$$

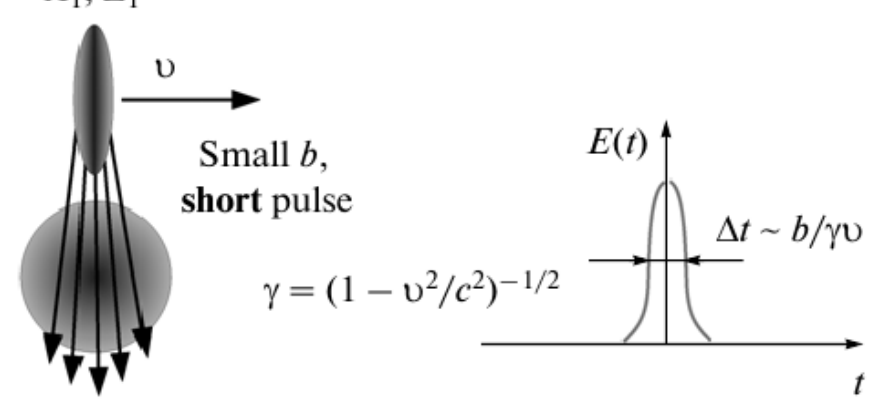

Target nucleus: $A_{2}, Z_{2}$

Projectile nucleus:

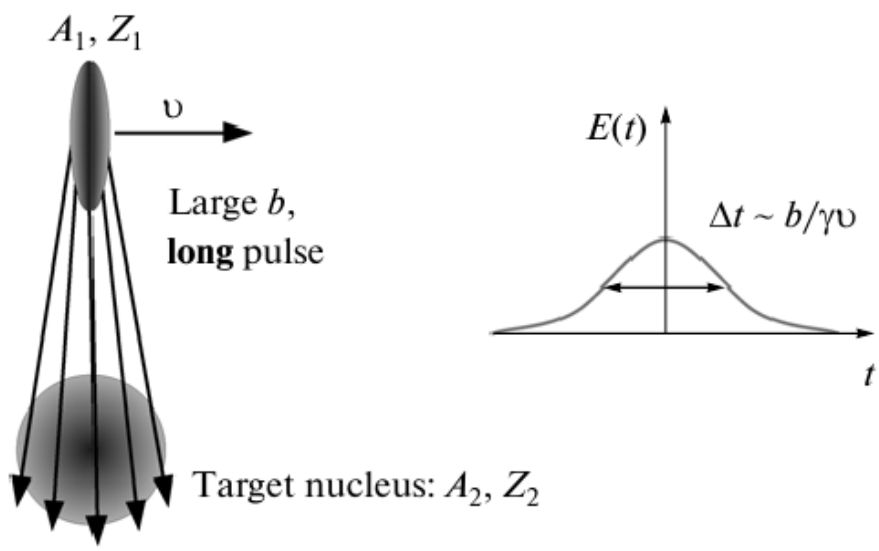

Figura 2.3: Influência do parâmetro de impacto $b$ sobre o espectro de energia dos fótons virtuais. Reproduzido da Referência [13].

O espectro de frequências ilustrado na Figura 2.2 contém todas as frequências até um máximo dado por $\omega_{\max } \sim 1 / \Delta t$ sendo $\Delta t \sim b / \gamma v$ a largura dos campos que formam o pulso $P_{1}$ [12]. Obtém-se que a energia máxima de um fóton do campo é dada por

$$
E_{\gamma}=\frac{\hbar}{\Delta t} \sim \frac{\gamma \hbar v}{b}
$$

Esta expressão vale tanto para o referêncial do alvo quanto para o referencial do labora- 
tório, que no caso de um colisor, é o referencial do centro de massa em colisões AA ou pp. É importante ressaltar que este trabalho sempre se referirá ao referencial do alvo e assim se deve interpretar $E_{\gamma}$ e o fator de Lorentz $\gamma$. Conhecido o fator de Lorentz, $\gamma_{L}$, no referencial do laboratório, pode-se obter $\gamma$ pela relação $\gamma=2 \gamma_{L}^{2}-1$.

\subsubsection{Probabilidade de Sobrevivência}

Como já mencionado, faz-se uso extensivo na literatura de UPCs da aproximação do núcleo por uma esfera ao se considerar $b_{\min }=R_{1}+R_{2}$. Sabe-se, no entanto, que a matéria nuclear apresenta uma densidade variável, em particular, aproximadamente constante no interior do núcleo passando a decrescente em direção à superfície. Esse conhecimento é obtido por meio de espalhamento de elétrons a fim de se deduzir, de fato, a densidade de carga. A equação empírica $R=r_{0} A^{1 / 3}$ fornece o raio nuclear à metade da densidade.

Uma maneira mais realista de tratar o caráter difuso da superfície nuclear no contexto de colisões periféricas é calcular a probabilidade de sobrevivência dos íons ao passarem um pelo outro em qualquer parâmetro de impacto.

A probabilidade de sobrevivência é definida como

$$
T(b)=\exp [-2 \chi(b)]
$$

e pode ser calculada de várias formas. Assumindo-se que colisões binárias nucleon-nucleon levam à fragmentação nuclear ou a outro processo hadrônico, pode-se mostrar que [14]

$$
\chi(b)=\frac{\sigma_{N N}}{4 \pi} \int_{0}^{\infty} d q q \tilde{\rho_{t}}(q) \tilde{\rho_{p}}(q) J_{0}(q b)
$$

onde $\sigma_{N N}$ é a seção de choque total nucleon-nucleon, $\tilde{\rho}_{t(p)}(q)$ é a transformada de Fourier da densidade nuclear do alvo (projétil) e $J_{0}$ é a função cilíndrica de Bessel de ordem zero. Para as energias atingidas neste estudo se utilizou $\sigma_{N N}=80 \mathrm{mb}$ para o caso $\mathrm{PbPb}$ e $\sigma_{N N}=53 \mathrm{mb}$ para o caso AuAu [15].

A distribuição de Woods-Saxon da densidade nuclear é bem descrita por uma convolução 
de uma esfera rígida e uma função de Yukawa, de modo que a transformada de Fourier da densidade nuclear pode ser calculada analiticamente como [16]

$$
\tilde{\rho}(q)=\frac{4 \pi \rho_{0}}{q^{3}}\left[\sin \left(q R_{0}\right)-q R_{0} \cos \left(q R_{0}\right)\right] \frac{1}{1+q^{2} a_{Y}^{2}}
$$

com $\rho_{0}=0.16 \mathrm{fm}^{-3}$ e $R_{0}=6.62 \mathrm{fm}$ para Pb. Para o Au temos $\rho_{0}=0.17 \mathrm{fm}^{-3}$ e $R_{0}=6.43$ fm. O parâmetro da função de Yukawa é $a_{Y}=0.7 \mathrm{fm}$. A expressão (2.14) é um dos possíveis fatores de forma nucleares.

No caso de uma colisão $\mathrm{Pb}-\mathrm{Pb}$, a probabilidade de sobrevivência tem o comportamento apresentado pela Figura 2.4 .

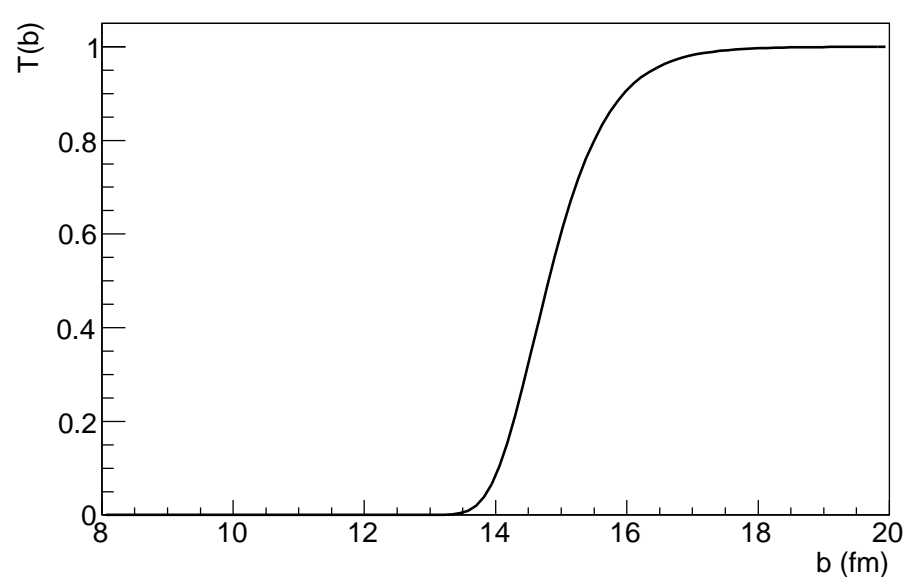

Figura 2.4: Probabilidade de sobrevivência de íons em uma colisão $\mathrm{Pb}-\mathrm{Pb}$.

Fica evidente pela Figura 2.4 que existe uma probabilidade maior do que zero de que os núcleos sobrevivam à colisão ainda que eles se perpassem a um parâmetro de impacto pouco menor do que $2 R_{P b}$. Além disso, a probabilidade de sobrevivência dos íons acima de $b=2 R_{P b}$ não é $100 \%$ senão para $b \sim 18 \mathrm{fm}$.

\subsubsection{Seção de Choque em Colisões Ultra-periféricas}

O cálculo da seção de choque nessa categoria de colisão entre íon pesados é de fato o objetivo final do método acima descrito, como era o objetivo de Fermi em seu estudo de excitação e 
ionização atômica. As Equações (2.10) e (2.12) são, então combinadas e reescritas na forma

$$
n\left(E_{\gamma}, b\right)=\frac{Z_{1}^{2} \alpha}{\pi^{2}} \frac{1}{\beta^{2} b^{2}} x^{2}\left[K_{1}^{2}(x)+\frac{1}{\gamma^{2}} K_{0}^{2}(x)\right] \exp [-2 \chi(b)]
$$

Aqui foi suprimido o $E_{\gamma}$ no denominador, de modo que $n\left(E_{\gamma}, b\right)$ representa agora apenas número de fótons equivalentes para certo parâmetro de impacto, lembrando que permanece válido $x=\frac{E_{\gamma} b}{\hbar \gamma \beta c}$. Essa forma adimensional é útil para escrever a seção de choque na forma geral como é usualmente apresentada

$$
\sigma_{X}=\int \frac{d E_{\gamma}}{E_{\gamma}} N\left(E_{\gamma}\right) \sigma_{\gamma A \rightarrow X}\left(E_{\gamma}\right)
$$

onde $\sigma_{\gamma A \rightarrow X}$ é a seção de choque do processo fotonuclear devido a um fóton real, $X$ é o produto de interesse na reação e $N\left(E_{\gamma}\right)$ é dado por

$$
N\left(E_{\gamma}\right)=2 \pi \int_{0}^{\infty} n\left(E_{\gamma}, b\right) b d b
$$

Note-se que com a inclusão da probabilidade de sobreviência no fluxo de fótons equivalentes, a integral no espaço de todos os parâmetros de impacto possíveis pode ser feita no intervalo $[0, \infty]$. Além disso, deve ser feita numericamente e a forma final do fluxo pode vista na Figura 2.5 para uma colisão $\mathrm{PbPb}$ a $\sqrt{s_{N N}}=2.76 \mathrm{TeV}$.

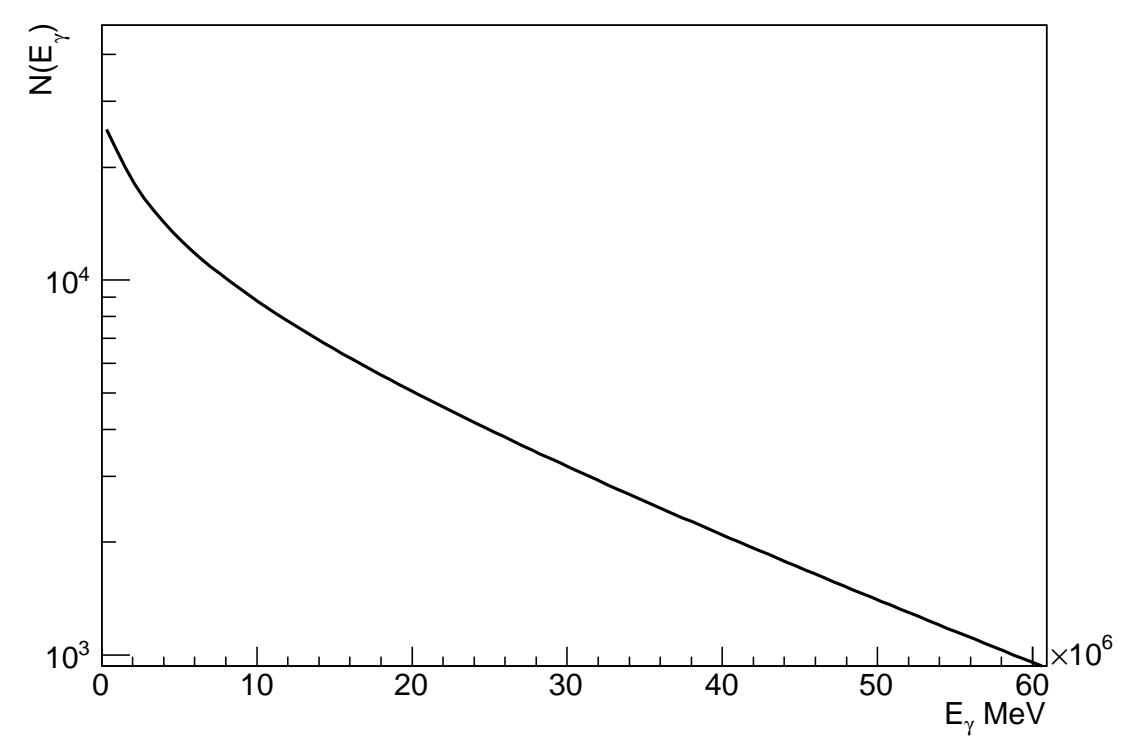

Figura 2.5: Fluxo de fótons virtuais em uma colisão $\mathrm{PbPb}$ a $\sqrt{s_{N N}}=2.76 \mathrm{TeV}$. 
Em colisões entre dois íons idênticos, o resultado da Equação (2.16) ainda deve incluir um fator 2 dada a simetria do problema.

Não é incomum, no entanto, a necessidade de representar os resultados de seção de choque em termos da rapidez, ou rapidity em inglês, das partículas produzidas. A rapidez de uma partícula é definida em termos de sua energia e seu momento longitudinal. Neste caso,

$$
y=\frac{1}{2} \ln \left(\frac{E+p_{z}}{E-p_{z}}\right)
$$

onde $p_{z}$ é precisamente a componente do momento na direção do feixe.

Uma relação bastante simples entre a distribuição em rapidez e a distribuição de energia é $\frac{d \sigma}{d y}=E_{\gamma} \frac{d \sigma}{d E_{\gamma}}$ [17]. Sendo assim, uma vez identificada a seção de choque diferencial como sendo o integrando da Equação (2.16)

$$
\frac{d \sigma_{\gamma A \rightarrow A P}}{d E_{\gamma}}=\frac{N\left(E_{\gamma}\right)}{E_{\gamma}} \sigma_{\gamma A \rightarrow A P}\left(E_{\gamma}\right),
$$

pode-se escrever

$$
\frac{d \sigma_{\gamma A \rightarrow A P}}{d y}=E_{\gamma} \frac{d \sigma_{\gamma A \rightarrow A P}}{d E_{\gamma}}
$$

o que leva a

$$
\frac{d \sigma_{\gamma A \rightarrow A P}}{d y}=N\left(E_{\gamma}\right) \sigma_{\gamma A \rightarrow A P}\left(E_{\gamma}\right)
$$

Nas equações acima, $P$ representa uma partícula de interesse.

Uma relação entre a energia do fóton e a rapidez da partícula produzida deve existir a fim de que a Equação (2.21) tenha real utilidade. Usando-se o fato de que a energia da partícula produzida é muito próxima da energia do fóton que lhe deu origem, pode-se reescrever a definção (2.18) como

$$
y=\ln \left[\frac{E_{\gamma}}{\gamma_{L} M_{P}}\right] \text {, }
$$

onde $E_{\gamma}$ corresponde à energia do fóton no referencial do alvo, $\gamma_{L}$ é o fator de contração de Lorentz dos feixes no colisor e $M_{P}$ é a massa da partícula de interesse. Levou-se em conta que 
$2 k \gamma_{L}=E_{\gamma}$, sendo $\gamma_{L}=\sqrt{s_{N N}} / 2 m_{p}$, onde $k$ é a energia do fóton no referencial do laboratório e $m_{p}$ é a massa do próton.

Finalmente, a seção de choque diferencial em termos da rapidez da partícula $P$ produzida para colisões AA é dada por

$$
\frac{d \sigma_{A A \rightarrow A A P}(y)}{d y}=\frac{d \sigma_{\gamma A \rightarrow A P}(y)}{d y}+\frac{d \sigma_{\gamma A \rightarrow A P}(-y)}{d y} .
$$

Em colisões ultra-periféricas, processos $\gamma A$ não são os únicos possíveis. Também são possíveis processos $\gamma \gamma \rightarrow X$. Em eventos de energias tão altas quanto as praticadas no LHC, processos eletrofracos tais como $\gamma \gamma \rightarrow W^{+} W^{-}$podem ser estudados. A seção de choque em processos dessa natureza é calculada como

$$
\sigma_{X}=\int \frac{d E_{\gamma 1}}{E_{\gamma 1}} \frac{d E_{\gamma 2}}{E_{\gamma 2}} N\left(E_{\gamma 1}\right) N\left(E_{\gamma 2}\right) \sigma_{X}^{\gamma \gamma}\left(E_{\gamma 1}, E_{\gamma 2}\right)
$$

Processos fóton-fóton não fazem parte dos objetivos deste trabalho.

\subsection{Teoria de Regge}

A teoria de Regge é uma teoria para interações hádron-hádron. Sua aplicabilidade à descrição de interações fóton-hádron em altas energias se explica pelo fato de que o fóton energético inicialmente flutua para um par $q \bar{q}$ o qual então interage com o hádron alvo por meio da força nuclear forte. Esta interação se dá, no entanto, não por meio de uma partícula virtual com spin definido mas sim por meio de uma família de ressonâncias. Tal família é conhecida como trajetória de Regge e é comumente chamada de reggeon.

A duração da flutuação é determinada pelo princípio de incerteza. Para um fóton de virtualidade $Q$ flutuando para um estado de massa $M_{V}$, a duração da flutuação é da ordem de

$$
\Delta t \approx \frac{\hbar}{\sqrt{M_{V}^{2} c^{4}+Q^{2} c^{2}}} \approx \frac{\hbar}{M_{V} c^{2}}
$$

A última aproximação é sempre válida em colisores de hádrons visto que a virtualidade dos 
fótons é baixa [5]. A função de onda do fóton é escrita como uma decomposição de Fock

$$
|\gamma\rangle=C_{\text {bare }}\left|\gamma_{\text {bare }}\right\rangle+C_{\rho}\left|\gamma_{\rho}\right\rangle+C_{\omega}\left|\gamma_{\omega}\right\rangle+C_{\phi}\left|\gamma_{\phi}\right\rangle+\ldots+C_{q \bar{q}}\left|\gamma_{q \bar{q}}\right\rangle
$$

Após a interação o par $q \bar{q}$ finalmente emerge como um méson vetorial.

Explicada a motivação, é importante esclarecer que esta seção se dedicará a expor, de maneira consistente porém não exaustiva, alguns aspectos importantes da teoria de Regge, em particular aqueles essenciais à compreensão da próxima seção, centrada na foto-produção de mésons vetoriais. De igual forma, alguns conceitos em colisões relativísticas serão tocados.

\subsubsection{Trajetórias de Regge e Amplitude de Espalhamento}

Um conceito interessante na teoria é o de função trajetória, $\alpha_{n}(s)$, onde $s$ é o quadrado da energia do centro de massa e o índice $n$ identifica uma partícula. O modelo de Regge estabelece a existência de funções que correlacionam partículas, ou ressonâncias, por meio de seus números quânticos. Partículas com os mesmos números quânticos internos (número bariônico, isospin, paridade, estranheza, etc) estão correlacionados por uma mesma e única função $\alpha_{n}(s)$ tal que partículas diferentes diferem sempre em 2 unidades de spin.

Como exemplo, considere-se um nucleon, seja proton ou neutron, identificado apenas como $\mathrm{N}(938)$ com isospin 1/2, paridade + e spin 1/2. As ressonânias $\mathrm{N}(1688)$ e $\mathrm{N}(2220)$, do sistema $\pi N$, possuem o mesmo isospin e a mesma paridade do nucleon porém spin, respectivamente, $5 / 2$ e 9/2. Há então uma trajetória de Regge tal que

$$
\begin{aligned}
& \alpha_{N}\left(0.938^{2}\right)=1 / 2 \\
& \alpha_{N}\left(1.688^{2}\right)=5 / 2 \\
& \alpha_{N}\left(2.220^{2}\right)=9 / 2
\end{aligned}
$$

Ou seja, dada a massa invariante, a função trajetória localiza o spin correspondente dentro de uma sequência de partículas que possuam os mesmos número quânticos internos. A Figura 2.6 ilustra não apenas a trajetória acima mas também aquela referente a ressonância $\Delta(1236)$. 


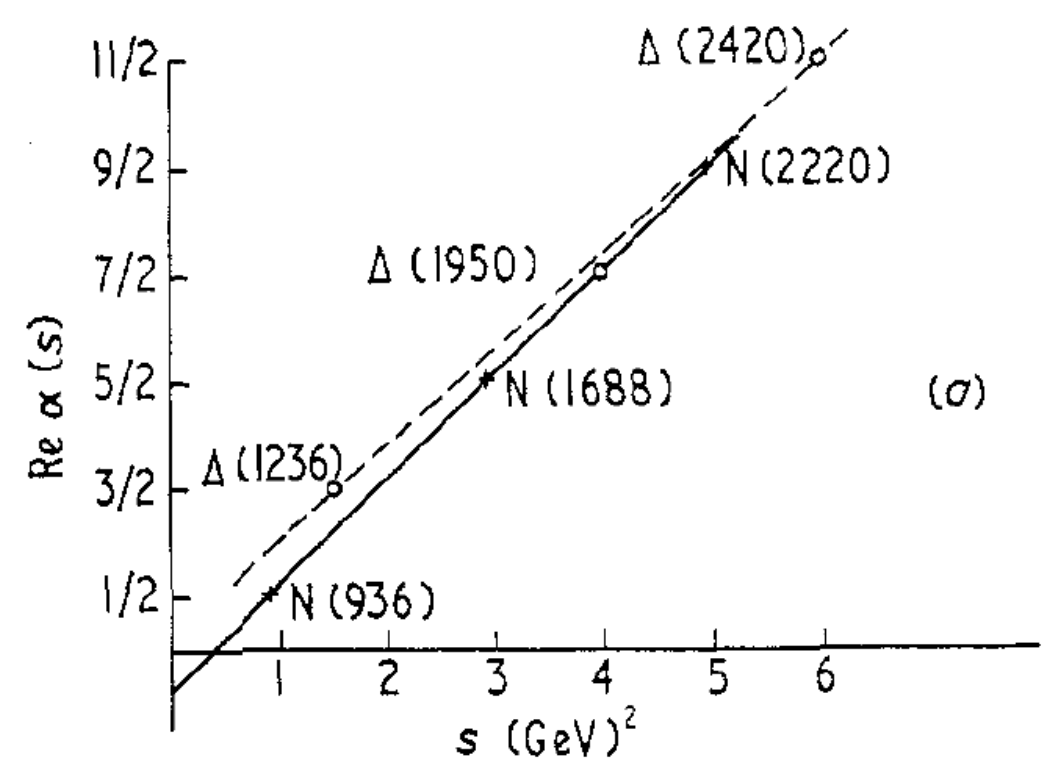

Figura 2.6: Exemplos de traetórias de Regge. Extraído da Referência [18].

Ambas as trajetórias da figura são aparentemente retas. De fato, esse comportamento próximo ao linear se verificará para todos os demais casos, e não apenas isso, mas a inclinação de qualquer trajetória de Regge é aproximadamente 1. De modo que para a maior parte das aplicações, costuma-se assumir que

$$
\alpha_{n}(s)=\alpha_{n}^{0}+s
$$

Onde $\alpha_{n}^{0}$ naturalmente depende de cada caso. No exemplo da Figura temos $\alpha_{N}^{0}=-0.37$ e $\alpha_{\Delta}^{0}=0.15$

Antes de continuar, é importante lembrar algumas definições e conceitos relativos à colisões em altas energias.

Primeiramente, uma colisão relativística pode ser esquematizada como na Figura 2.7.

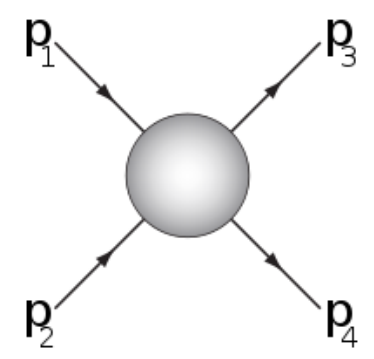

Figura 2.7: Esquema para uma colisão relativística. 
Considerando que se use uma métrica de Minkowski em que a diagonal seja dada por $(1,-1,-1,-1)$, as variáveis de Mandelstam então podem ser escritas como

$$
\begin{aligned}
& s=\left(p_{1}+p_{2}\right)^{2}=\left(p_{3}+p_{4}\right)^{2} \\
& t=\left(p_{1}-p_{3}\right)^{2}=\left(p_{2}-p_{4}\right)^{2} \\
& u=\left(p_{1}-p_{4}\right)^{2}=\left(p_{2}-p_{3}\right)^{2}
\end{aligned}
$$

Além disso, é comum encontrar referências aos chamados canais s, t e u. Ocorre que em Teoria Quântica de Campos, a absorção de uma partícula de quadri-momento p corresponde à emissão de uma anti-partícula de quadri-momento -p. Além disso, a amplitude de espalhamento, $A(s, t)$, para o cálculo da seção de choque em altas energias é, como se vê, uma função das variáveis de Mandelstam $s$ e $t$. Como ambas são invariantes cinemáticas pela definição, essa amplitude descreve, na verdade qualquer um do seguintes processos de espalhamento:

$$
\begin{aligned}
& 1.1+2 \rightarrow 3+4 \\
& 2.1+\overline{3} \rightarrow \overline{2}+4 \\
& 3.1+\overline{4} \rightarrow \overline{2}+3
\end{aligned}
$$

os quais estão ilustrados na Figura 2.8. Esta propriedade de reações cruzadas é às vezes referenciada na literatura como crossing symmetry.

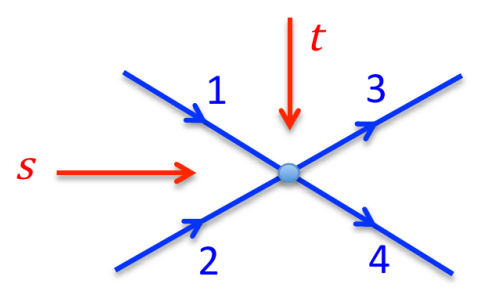

(a) processo 1

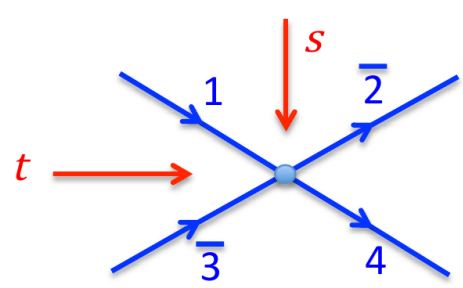

(b) processo 2

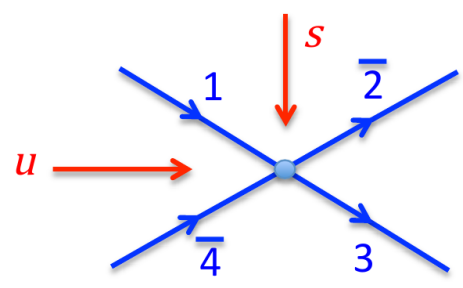

(c) processo 3

Figura 2.8: Esquemas para uma colisão relativística genérica representando os canais s, t e u.

Em cada processo, um invariante cinemático assume o papel da massa invariante, ou energia do centro de massa da colisão. Finalmente, em teoria de espalhamento em altas energias, podese escrever a amplitude $A(s, t)$ como [19]

$$
A(s, t)_{s \rightarrow \infty} \approx \sum_{n} \eta_{n}(t) \beta_{n}(t)\left(\cos \theta_{t}\right)^{\alpha_{n}(t)}
$$


onde $\theta_{t}$ é o ângulo de espalhamento no canal t e $\alpha_{n}(t)$ é uma trajetória de Regge em função de t o qual, vale notar, além de ser a massa invariante do canal t, é também o momento transferido com respeito ao canal s e tal nomeclatura é recorrente na literatura.

\subsection{Foto-produção de Mésons Vetoriais}

Os mecanismos de foto-produção já se encontram inseridos no código CRISP por meio da foto-absorção, bem como os canais de interação de estado final. Ambos foram tema de trabalhos anteriores e mais detalhes podem ser encontrados em [6,20]. Os aspectos mais importantes dos modelos usados e do método de cálculo empregado são descritos a seguir.

O processo de foto-produção de mésons vetoriais é considerado de acordo como o modelo universal de soft dipole Pomeron proposto por Martynov, Predazzi and Prokudin [19, 21]. O Pomeron é um reggeon cuja trajetória de Regge intercepta o eixo dos spins em exatamente 1, ou seja, $\alpha_{P}(0)=1$, e particularmente neste modelo, foge-se à regra e a trajetória de Regge não é linear mas dada por

$$
\alpha_{P}(t)=1+\gamma\left(\sqrt{4 m_{\pi}^{2}}-\sqrt{4 m_{\pi}^{2}-t}\right)
$$

onde $\gamma$ é um parâmetro ajustável e $m_{\pi}$ é a massa do píon.

As amplitudes são calculadas de acordo com o Modelo de Dominância Vetorial (VDM na sigla em Inglês) [22, 23].

Com este modelo é possível reproduzir todos os dados de foto-produção em prótons dos mésons $\rho, \omega, \phi$ e $\mathrm{J} / \psi$ na região $1.7 \leq \sqrt{s} \leq 250 \mathrm{GeV}$, para virtualidades $0 \leq Q^{2} \leq 35 \mathrm{GeV}^{2}$ e para valores de momento transferido $|t|<1.6 \mathrm{GeV}^{2}$. O uso do modelo de troca de Pomeron é bem avalizado por experimentos em foto-produção de $\mathrm{J} / \psi$ usando o detector ZEUS [24] que sugere a descrição da foto-reação como um processo do tipo soft ao invés do uso de QCD pura.

Como já mencionado, o fóton flutua para um par $q \bar{q}$ por um tempo dado pela Equação (2.25). A duração desse estado, sua lifetime, é de fato grande o bastante para permitir que o par 
interaja com um próton pela troca de um Pomeron ou um reggeon secundário, e emerja como um méson vetorial.

No Modelo de Dominânica Vetorial a amplitude da foto-reação é dada em termos da amplitude do processo de espalhamento méson vetorial-nucleon conforme

$$
A_{\gamma p \rightarrow V p}=N_{C} N_{V} A_{V p \rightarrow V p}
$$

onde os coeficientes $N_{C}$ e $N_{V}$ podem ser calculados a partir das larguras de decaimento dos mésons vetoriais em $e^{-} e^{+}$assumindo simetria de sabor $S U(4)$ [21]. A amplitude de espalhamento do processo méson-nucleon é

$$
A\left(z, t, M_{V}^{2}, \tilde{Q}^{2}\right)=\mathbb{P}\left(z, t, M_{V}^{2}, \tilde{Q}^{2}\right)+f\left(z, t, M_{V}^{2}, \tilde{Q}^{2}\right)+\ldots
$$

onde $\tilde{Q}^{2}=M_{V}^{2}+Q^{2}$, sendo $M_{V}$ a massa do méson e $Q^{2}$ a virtualidade do fóton. Ainda, $z=\cos \theta_{t}$. $\mathbb{P}\left(z, t, M_{V}^{2}, \tilde{Q}^{2}\right)$ é a contribução tipo Pomeron e é dada por

$$
\mathbb{P}\left(z, t, M_{V}^{2}, \tilde{Q}^{2}\right)=i g_{0}\left(t, M_{V}^{2}, \tilde{Q}^{2}\right)(-i z)^{\alpha_{\mathbb{P}}(t)-1}+i g_{1}\left(t, M_{V}^{2}, \tilde{Q}^{2}\right) \ln (-i z)(-i z)^{\alpha_{\mathbb{P}}(t)-1}
$$

onde o primeiro termo da soma é a contribuição de pólo $j$ simples e o segundo a contribuição de um pólo $j$ duplo. O segundo termo do lado direito da Equação (2.34) tem a forma

$$
f\left(z, t, M_{V}^{2}, \tilde{Q}^{2}\right)=i g_{f}\left(t, M_{V}^{2}, \tilde{Q}^{2}\right)(-i z)^{\alpha_{f}(t)-1}
$$

O modelo contém 12 parâmetros livres que foram obtidos por meio do ajuste de 357 pontos experimentais para cada méson. O resultado desse ajuste pode ser visto na Figura 2.9.

A seção de choque diferencial para o processo $\gamma p \rightarrow V p$ fica assim dada por

$$
\frac{d \sigma}{d t}=4 \pi\left|N_{C} N_{V} A_{V p \rightarrow V p}\left(z, t, M_{V}^{2}, \tilde{Q}^{2}\right)\right|^{2}
$$

de onde se chega à seção de choque elástica

$$
\sigma\left(z, M_{V}^{2}, \tilde{Q}^{2}\right)_{\gamma p \rightarrow V p}=4 \pi \int_{t_{-}}^{t_{+}} d t\left|N_{C} N_{V} A_{V p \rightarrow V p}\left(z, t, M_{V}^{2}, \tilde{Q}^{2}\right)\right|^{2}
$$

onde $t_{-}$e $t_{+}$são os momentos transferidos determinados pela condição cinemática $-1 \leqslant \cos \theta_{s} \leqslant$ 


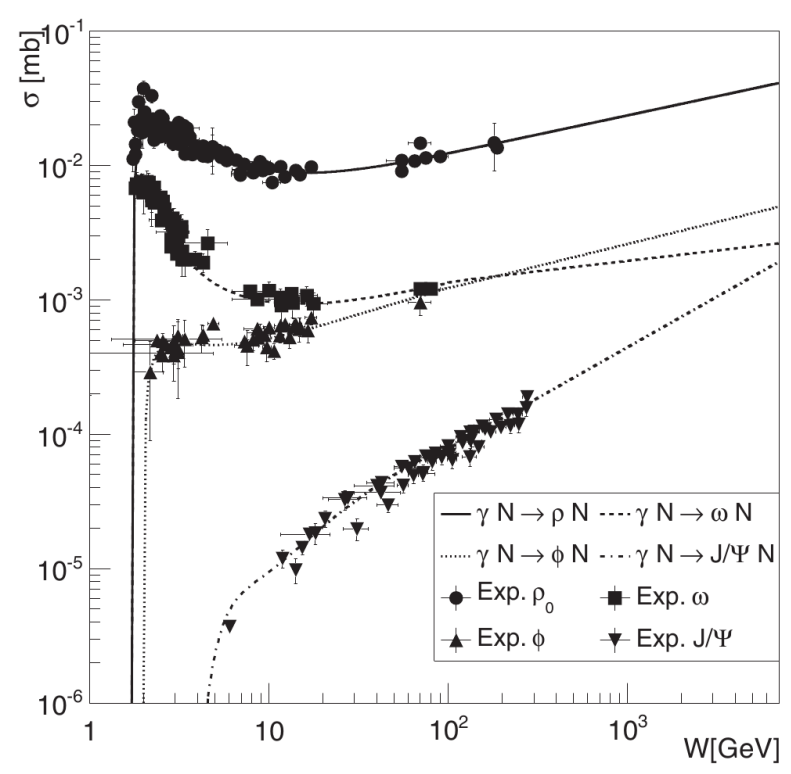

Figura 2.9: Seção de choque elástica de fotoprodução de mésons vetoriais em interação com proton. $W=\sqrt{s_{\gamma N}}$. Extraído de [6].

1 , sendo $\theta_{s}$ o ângulo de espalhamento no canal s da reação.

Acima de $W_{\gamma p}=100 \mathrm{GeV}$, sendo $W_{\gamma p}$ a energia do centro de massa do par $\gamma p$, a seção de choque $\gamma p \rightarrow V p$ foi parametrizada de acordo com funções contínuas simples como proposto na Referência [25],

$$
\begin{aligned}
\left.\frac{d \sigma^{\rho, \omega}}{d t}\right|_{t=0} & =b_{v}\left(X W_{\gamma p}^{\varepsilon}+Y W_{\gamma p}^{\eta}\right) \\
\left.\frac{d \sigma^{\phi, J / \psi}}{d t}\right|_{t=0} & =b_{v}\left(X W_{\gamma p}^{\varepsilon}\right)
\end{aligned}
$$

Os termos $X$ e $Y$ correspondem, respectivamente, às contribuições de Pomeron e troca de méson. O resultado obtido com o ajuste da Equações (2.39) para a região de energias distante do limiar de produção foram as equações a seguir:

$$
\begin{aligned}
\sigma(\gamma N \rightarrow \rho N) & =3.31 W^{0.28}+173.40 W^{-1.66} \\
\sigma(\gamma N \rightarrow \omega N) & =0.67 W^{0.15}-5901.63 W^{-2.70} \\
\sigma(\gamma N \rightarrow \phi N) & =0.27 W^{0.33} \\
\sigma(\gamma N \rightarrow J / \psi N) & =0.0024 W^{0.75}
\end{aligned}
$$

A energia do centro de massa é dada em GeV e a seção de choque em $\mu b$. 


\subsubsection{Interações de Estado Final}

Canais de interação dos mésons vetoriais com a matéria nuclear são essenciais à correta reprodução do ambiente do núcleo. Canais de interação de estado final (FSI, na sigla em inglês) para os mésons $\rho, \omega, \phi$ e $J / \psi$ também estão presentes no CRISP. Para os canais elásticos $V N \rightarrow V N$, as seções de choque foram calculadas de acordo com a Equação (2.38). Para os canais inelásticos foram usados os modelos de troca de um méson e modelo ressonante.

\section{Modelo de troca de um méson}

Este modelo foi empregado para o cálculo da FSI no caso dos mésons $\rho, \omega$ e $\phi$.

Para os canais $\omega N \rightarrow \pi N, \rightarrow \rho N$ e $\rightarrow \pi \pi N$ foi usado o modelo de troca de um bóson (EM) proposto por Likazov, Cassing, Sibirtsev e Rzjanin [26] que adota o modelo de dominância $\rho \omega \pi$ proposto por Gell-Mann, Sharp e Wagner [27]. Os diagramas de Feynman correspondentes a esses processos podem ser vistos na Figura 2.10, de b) a d).

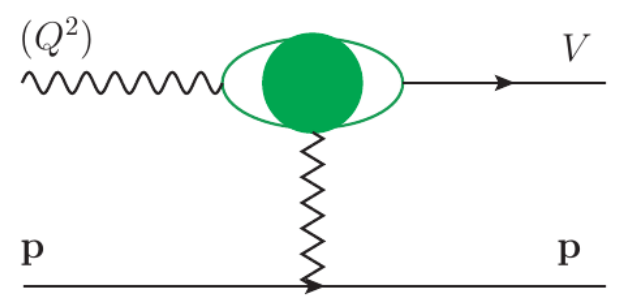

(a)

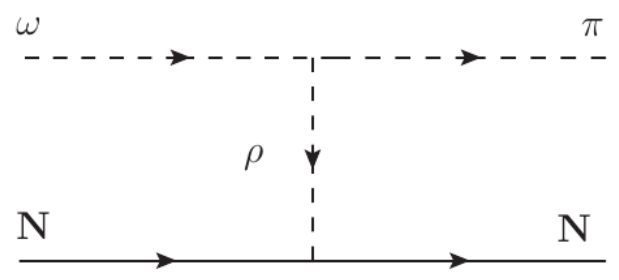

(c)

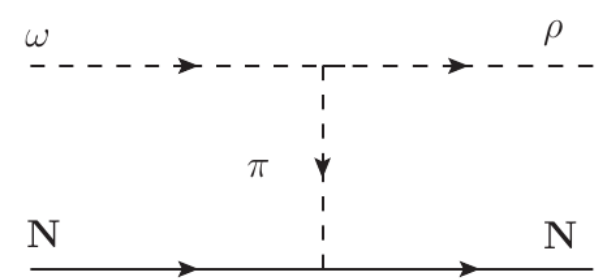

(b)

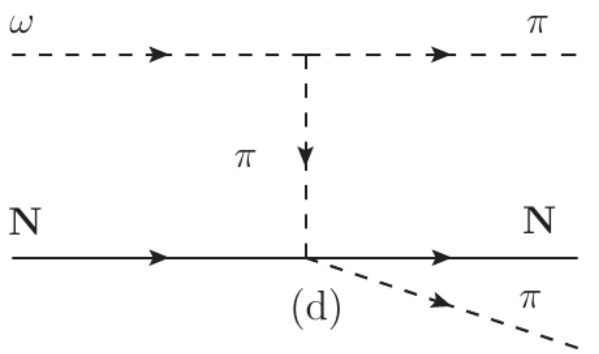

Figura 2.10: Diagramas de Feynman. a) foto-produção de méson vetorial. b) a d) FSI para o méson $\omega$. Extraído de [6].

A constante de acoplamento $g_{\omega \rho} \pi$ e o fator de forma são fixados pela reação $\pi N \rightarrow \omega N$ em comparação com dados experimentais. 
O modelo faz uso da Lagrangiana efetiva para a troca do méson $\mathrm{V}$,

$$
\mathscr{L}=\mathscr{L}_{\omega \rho \pi}+\mathscr{L}_{V N N},
$$

sendo a Lagrangiana de interação $\omega \rho \pi$ dada por

$$
\mathscr{L}_{\omega \rho \pi}=-\frac{g_{\omega \rho \pi}}{m_{\omega}} \varepsilon_{\alpha \beta \gamma \delta} \partial^{\alpha} \rho^{\beta} \partial^{\gamma} \omega^{\delta} \pi
$$

onde a constante de acoplamento $g_{\omega \rho \pi}=11.79$ é obtida da largura parcial do decaimento $\omega \rightarrow$ $3 \pi, \varepsilon_{\alpha \beta \gamma \delta}$ é o tensor anti-simétrico e $\omega, \rho$ e $\pi$, são os campos mesônicos correspondentes. As Lagrangianas de interação para os vértices $\rho N N$ e $\pi N N$ são dadas por

$$
\begin{aligned}
& \mathscr{L}_{\rho N N}=-g_{\rho N N}\left(\bar{N} \gamma^{\mu} \tau N \rho_{\mu}+\frac{\kappa}{2 m_{N}} \bar{N} \sigma^{\mu v} \tau N \partial_{\mu} \rho_{v}\right) \\
& \mathscr{L}_{\pi N N}=-g_{\pi N N} \bar{N} \gamma_{5} \tau N \cdot \pi,
\end{aligned}
$$

onde $\mathrm{N}$ representa o campo do nucleon, $\tau$ as matrizes de Pauli e $g_{\rho N N}=3.24$ é definido de acordo com a Referência [28]. A constante do tensor de acoplamento é dada pela razão $\kappa=$ $f_{\rho N N} / g_{\rho N N}=6.1$, enquanto $g_{\pi N N}=13.59$ [29]. Mais detalhes podem ser encontrados em [26].

A distribuição angular para duas partículas em colisão é calculada usando a seção de choque diferencial enquanto para a reação $\omega N \rightarrow \pi \pi N$ usa-se o gerador de eventos em espaço de fase do framework ROOT [30]. As reações inversas $\rho N \rightarrow \omega N$ e $\pi N \rightarrow \omega N$ também foram incluídas e são calculadas pelo método de balanço detalhado.

As seções de choque diferenciais obtidas a partir da Lagrangiana de interação para os canais calculados são apresentadas a seguir [26]:

- $\pi N \rightarrow \omega N$

$$
\begin{aligned}
& \frac{d \sigma}{d t}=\frac{g_{\omega \rho \pi}^{2}}{m_{\omega}^{2}} \frac{1}{8 \pi \lambda\left(s, m_{N}^{2}, m_{\pi}^{2}\right)} \frac{F_{\omega \rho \pi}^{2} F_{\rho N N}^{2}}{\left(t-m_{\rho}^{2}\right)^{2}} \\
& \times\left[-\left(g_{\rho N N}+f_{\rho N N}\right)^{2} m_{\omega}^{2} q_{\omega}^{2} t+\left(g_{\rho N N}^{2}-\frac{f_{\rho N N}^{2} t}{4 m_{N}^{2}}\right)\left(\frac{\sin ^{2} \theta}{8 s} \lambda\left(s, m_{N}^{2}, m_{\pi}^{2}\right) \lambda\left(s, m_{N}^{2}, m_{\omega}^{2}\right)\right)\right],
\end{aligned}
$$


onde

$$
q_{\omega}^{2}=\frac{\lambda\left(t, m_{\omega}^{2}, m_{\pi}^{2}\right)}{4 m_{\omega}^{2}}
$$

sendo $s$ o quadrado da energia do centro de massa da colisão, $t$ o quadri-momento transferido, $\theta$ o ângulo de emissão e $\lambda(x, y, z)$ a função triangular. Para o vértice $\omega \rho \pi$ é usado o fator de forma [26]

$$
F(t)=\frac{\Lambda^{2}-m_{\rho}^{2}}{\Lambda^{2}-t}
$$

Para o vértice $\rho N N$ se usa o fator de forma

$$
F(t, s)=\exp (\beta t) \exp (-\alpha s)
$$

O ajuste da seção de choque aos dados experimentais resultou nos seguintes valores dos parâmetros $\Lambda, \alpha$ e $\beta$ :

$$
\Lambda=2.7 \mathrm{GeV}, \quad \beta=2.3 \mathrm{GeV}^{-2}, \quad \alpha=0.16 \mathrm{GeV}^{-2}
$$

- $\omega N \rightarrow \rho N$

A seção de choque diferencial é dada por

$$
\frac{d \sigma}{d t}=-g_{\pi N N}^{2} \frac{g_{\omega \rho \pi}^{2}}{m_{\omega}^{2}} t \frac{\left(t-m_{\omega}^{2}-m_{\rho}^{2}\right)^{2}-4 m_{\omega}^{2} m_{\rho}^{2}}{96 \pi \lambda\left(s, m_{\omega}^{2}, m_{N}^{2}\right)} \times \frac{F_{\omega \rho \pi}^{2}(t) F_{\pi N N}^{2}(t)}{\left(t-m_{\pi}^{2}\right)^{2}} .
$$

onde a constante de acoplamento é $g_{\pi N N}=13.59$ [29].

- $\omega N \rightarrow \pi \pi N$

$$
\begin{aligned}
\sigma & =\frac{1}{32 \pi^{2} \lambda\left(s, m_{\omega}^{2}, m_{N}^{2}\right)} \int_{\left(m_{N}+m_{\pi}\right)^{2}}^{\left(\sqrt{s}-m_{\pi}\right)^{2}} d s_{1} \lambda^{1 / 2}\left(s_{1}, m_{\rho}^{2}, m_{N}^{2}\right) \\
& \times \sigma_{\rho N \rightarrow \pi N}\left(s_{1}\right) \int_{t_{-}}^{t_{+}} d t \frac{g_{\omega \rho \pi}^{2} F_{\omega \rho \pi}^{2}}{m_{\omega}^{2}\left(t-m_{\rho}^{2}\right)^{2}}\left[\left(t+m_{\omega}^{2}-m_{\pi}^{2}\right)^{2}-4 m_{\omega}^{2} t\right]
\end{aligned}
$$

onde $\sigma_{\rho N \rightarrow \pi N}$ é calculada pelo princípio de balanço detalhado da reação inversa. Esta seção de choque foi calculada usando o modelo ressonante que será descrita mais à frente. 
- $\omega N \rightarrow \omega N$

O canal elástico do méson $\omega$ é descrito pelo modelo de troca de $\sigma$ [26]. O modelo propõe as seguintes Lagrangianas de interação

$$
\begin{aligned}
& \mathscr{L}_{\sigma N N}=g_{\sigma N N} \bar{N} N \cdot \sigma, \\
& \mathscr{L}_{\omega \sigma \omega}=g_{\omega \sigma \omega}\left(\partial^{\alpha} \omega^{\beta} \partial_{\alpha} \omega_{\beta}-\partial^{\alpha} \omega^{\beta} \partial_{\beta} \omega_{\alpha}\right) \sigma,
\end{aligned}
$$

sendo as constantes de acoplamento escalares $g_{\sigma N N}=10.54$ e $g_{\omega \sigma \omega}=1.76$ e o fator de forma de monopolo dado por $\Lambda=2 \mathrm{GeV}$ o vértice definido por $\sigma N N$. A seção de choque diferencial pode ser então calculada por [26]

$$
\frac{d \sigma}{d t}=\frac{g_{\sigma N N}^{2} g_{\omega \sigma \omega}^{2}}{16 \pi m_{\omega}^{2} \lambda\left(s, m_{\omega}^{2}, m_{N}^{2}\right)}\left(4 m^{2}-t\right) \frac{F_{\sigma N N}^{2} F_{\omega \sigma \omega}^{2}}{\left(t-m_{\sigma}^{2}\right)^{2}}\left(m_{\sigma}^{4}-\frac{m_{\omega}^{2}}{3}+\frac{t^{2}}{12}\right)
$$

\section{Modelo de troca de um méson para o méson $J / \psi$}

O méson $J / \psi$ se dissocia em interação com a matéria nuclear como descrito nos diagramas da Figura 2.11.

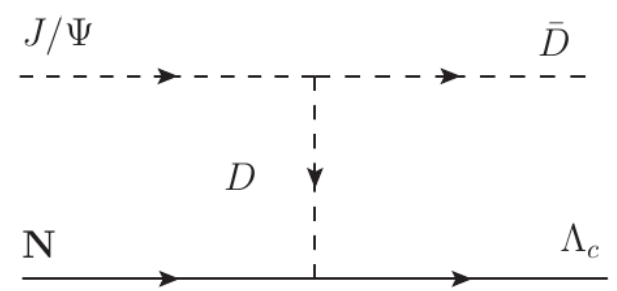

(a)

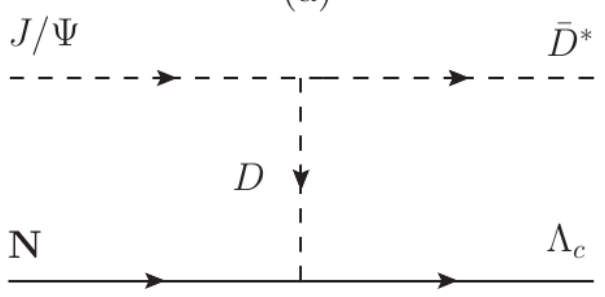

(c)

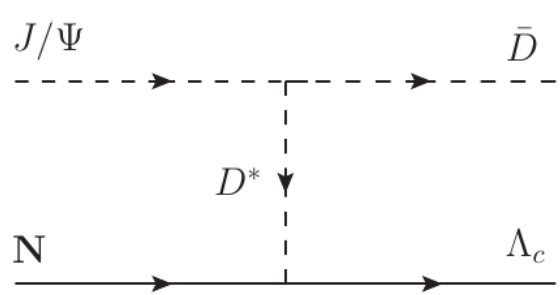

(b)

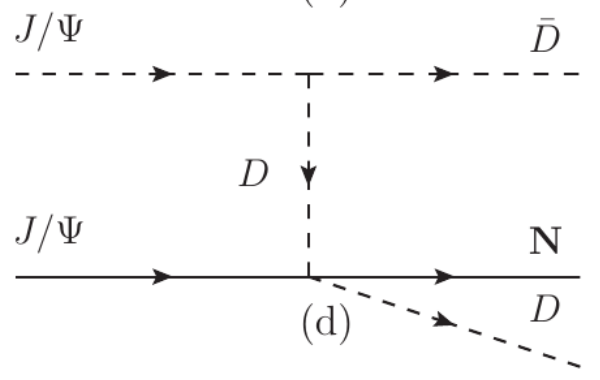

Figura 2.11: Diagramas de dissociação do méson $J / \psi[6]$.

A dissociação ocorre por meio das reações $J / \psi N \rightarrow \Lambda_{c} \bar{D}, \rightarrow \Lambda_{c} \bar{D}^{*}, \rightarrow N D \bar{D}$. A inclusão desses canais foi feita usando o modelo hadrônico de troca de um bóson proposto por Sibirtsev, Tsushima e Thomas [31]. O modelo é sustentado por medidas feitas pela Colaboração NA50 
no estudo de supressão de $J / \psi$ em colisões $\mathrm{Pb}-\mathrm{Pb}$ no Super Proton Synchrotron no CERN [32].

De acordo com o modelo, as densidades das Lagrangianas de interação são

$$
\begin{aligned}
\mathscr{L}_{J D D} & =i g_{J D D} J^{\mu}\left[\bar{D}\left(\partial_{\mu} D\right)-\left(\partial_{\mu} \bar{D}\right) D\right] \\
\mathscr{L}_{D N \Lambda_{c}} & =i g_{D N \Lambda_{c}}\left[\bar{N} \gamma_{5} \Lambda_{c} D+\bar{D} \bar{\Lambda}_{c} \gamma_{5} N\right] \\
\mathscr{L}_{J D^{*} D} & =\frac{g_{J D^{*} D}}{m_{J}} \varepsilon_{\alpha \beta \mu \nu}\left(\partial^{\alpha} J^{\beta}\right)\left[\left(\partial^{\mu} \bar{D}^{* \nu}\right) D+\bar{D}\left(\partial^{\mu} D^{* v}\right)\right] \\
\mathscr{L}_{D^{*} N \Lambda_{c}} & =-g_{D^{*} N \Lambda_{c}}\left[\bar{N} \gamma_{\mu} \Lambda_{c} D^{* \mu}+\bar{D}^{* \mu} \bar{\Lambda}_{c} \gamma_{\mu} N\right]
\end{aligned}
$$

com

$$
N=\left(\begin{array}{c}
p \\
n
\end{array}\right), \quad \bar{N}=N^{\dagger} \gamma_{0}, \quad D \equiv\left(\begin{array}{c}
D^{0} \\
D^{+}
\end{array}\right), \quad \bar{D}=D^{\dagger}
$$

Para $D^{*}$ e $\bar{D}^{*}$ uma notação similar é usada. Os vértices de interação são caracterizados pelas constantes de acoplamento $g_{J D D^{*}}=7.64, g_{D N \Lambda_{c}}=14.8, g_{D^{*} N \Lambda_{c}}=-19$ e por um fator de forma associado parametrizado na forma convencional de monopolo:

$$
F(t)=\frac{\Lambda^{2}}{\Lambda^{2}-t}
$$

$\Lambda$ é o parâmetro de corte, e é explicitamente definido como $\Lambda=3.1 \mathrm{GeV}$ para os vértices $J D D$ e $J D D^{*}$ enquanto para os vértices $D N \Lambda_{c}$ e $D^{*} N \Lambda_{c}$ é $\Lambda=2$.

As seções de choque diferenciais para as interações nucleon- $J / \psi$ representadas na Figura 2.11 são dadas por

$$
\begin{aligned}
\frac{d \sigma_{a, b}}{d \Omega} & =\frac{1}{64 \pi^{2} s}{\overline{\left|M_{a, b}\right|}}^{2}\left(\frac{\left[\left(m_{\Lambda}+m_{D}\right)^{2}-s\right]\left[\left(m_{\Lambda}-m_{D}\right)^{2}-s\right]}{\left[\left(m_{N}+m_{J / \psi}\right)^{2}-s\right]\left[\left(m_{N}-m_{J / \psi}\right)^{2}-s\right]}\right)^{1 / 2} \\
\frac{d \sigma_{c}}{d \Omega} & =\frac{1}{64 \pi^{2} s}{\overline{\left|M_{c}\right|}}^{2}\left(\frac{\left[\left(m_{\Lambda}+m_{D^{*}}\right)^{2}-s\right]\left[\left(m_{\Lambda}-m_{D^{*}}\right)^{2}-s\right]}{\left[\left(m_{N}+m_{J / \psi}\right)^{2}-s\right]\left[\left(m_{N}-m_{J / \psi}\right)^{2}-s\right]}\right)^{1 / 2}
\end{aligned}
$$

sendo ${\overline{\mid M_{a, b, c}}}^{2}$ são as amplitudes correspondentes a cada processo ao quadrado. As amplitudes são médias sobre os spins iniciais e somadas sobre os spins finais, e são dadas por 
[31]

${\overline{\mid M_{a}}}^{2}=\frac{8 g_{J D D}^{2} g_{D N \Lambda_{c}}^{2}}{3 m_{J / \psi}^{2}}\left(\frac{1}{q^{2}-m_{D}^{2}}+\frac{1}{2 p_{J / \psi} \cdot p_{\bar{D}}}\right)^{2}\left(p_{N} \cdot p_{\Lambda_{c}}-m_{N} m_{\Lambda_{c}}\right)\left[\left(p_{J / \psi} \cdot p_{\bar{D}}\right)^{2}-m_{J / \psi}^{2} m_{\bar{D}}^{2}\right]$

$$
\begin{aligned}
& {\overline{\left|M_{b}\right|}}^{2}=\frac{g_{J D^{*} D}^{2} g_{D^{*} N \Lambda_{c}}^{2}}{3 m_{J / \psi}^{2}} \frac{1}{\left(q^{2}-m_{D^{*}}^{2}\right)^{2}} \\
& \times\left\{m_{J / \psi}^{2}\left[p^{2} q^{2}-\left(m_{\Lambda_{c}}^{2}-m_{N}^{2}\right)^{2}\right]\right. \\
& +2\left(p_{J / \psi} \cdot p\right)\left(p_{J / \psi} \cdot q\right)\left(m_{\Lambda_{c}}^{2}-m_{N}^{2}\right)-p^{2}\left(p_{J / \psi} \cdot q\right)^{2} \\
& \left.-q^{2}\left(p_{J / \psi} \cdot p\right)^{2}-4\left(p_{N} \cdot p_{\Lambda_{c}}-m_{N} m_{\Lambda_{c}}\right)\left[m_{J / \psi}^{2} q^{2}-\left(p_{J / \psi} \cdot p\right)^{2}\right]\right\} \\
& {\overline{\mid M_{c}}}^{2}=\frac{4 g_{J D^{*} D}^{2} g_{D N \Lambda_{c}}^{2}}{3 m_{J / \psi}^{2}} \frac{1}{\left(q^{2}-m_{D}^{2}\right)^{2}}\left(p_{N} \cdot p_{\Lambda_{c}}-m_{N} m_{\Lambda_{c}}\right)\left[\left(p_{J / \psi} \cdot p_{\bar{D}^{*}}\right)^{2}-m_{J / \psi}^{2} m_{\bar{D}^{*}}^{2}\right],
\end{aligned}
$$

onde $q \equiv p_{J / \psi}-p_{\bar{D}}=p_{\Lambda_{c}}-p_{N}$ denota o quadri-momento transferido e $p \equiv p_{\Lambda_{c}}+p_{N}$.

A seção de choque diferencial dupla para a reação $J / \psi+N \rightarrow N+D+\bar{D}$ é escrita como

$$
\frac{d^{2} \sigma_{d}}{d t d s_{1}}=\frac{g_{J / \psi D D}^{2}}{96 \pi^{2} q_{J / \psi}^{2} s} q_{D} \sqrt{s_{1}} \frac{F^{2}(t)}{\left(t-m_{D}^{2}\right)^{2}} \frac{\left[\left(m_{J / \psi}+m_{D}\right)^{2}-t\right]\left[\left(m_{J / \psi}-m_{D}\right)^{2}-t\right]}{m_{J / \psi}^{2}} \sigma_{D N}\left(s_{1}\right)
$$

onde

$$
\begin{aligned}
q_{D}^{2} & =\frac{\left[\left(m_{N}+m_{D}\right)^{2}-s_{1}\right]\left[\left(m_{N}-m_{D}\right)^{2}-s_{1}\right]}{4 s_{1}}, \\
q_{J / \psi}^{2} & =\frac{\left[\left(m_{N}+m_{J / \psi}\right)^{2}-s\right]\left[\left(m_{N}-m_{J / \psi}\right)^{2}-s\right]}{4 s} .
\end{aligned}
$$

As seções de choque totais $D N$ e $\bar{D} N$ podem ser parametrizadas como [33]

$$
\sigma_{\bar{D} N}\left(s_{1}\right)=\left(\frac{\left[\left(m_{\Lambda_{c}}+m_{\pi}\right)^{2}-s_{1}\right]\left[\left(m_{\Lambda_{c}}-m_{\pi}\right)^{2}-s_{1}\right]}{\left[\left(m_{N}+m_{D}\right)^{2}-s_{1}\right]\left[\left(m_{N}-m_{D}\right)^{2}-s_{1}\right]}\right)^{1 / 2} \frac{27}{s_{1}}+20
$$

$\operatorname{com} m_{\Lambda_{c}}$ e $m_{\pi}$ sendo as massas do hyperon e do píon, respectivamente, dadas em GeV. A seção de choque é calculada em $m b$. 
Modelo ressonante: $\rho, \omega$ e $\phi$

Para a inclusão dos canais de interação $\pi N \rightarrow \rho N, \omega N$ e $\phi N$, são utilizados os melhores resultados de ajuste obtidos com o modelo ressonante como feito por Sibirtsev, Cassing e Mosel [34]. Neste modelo, a seção de choque de produção induzida por píons dos mésons $\rho, \omega$ e $\phi$ é calculada para uma reação do tipo

$$
\pi+N \rightarrow R \rightarrow M+N
$$

onde $R$ é uma ressonância bariônica. Assumindo que o elemento de matriz ao quadrado é proporcional a uma função Breit-Wigner, a seção de choque pode ser calculada como

$$
\sigma_{\pi+N \rightarrow M+N}(s)=\frac{\pi}{k^{2}} \frac{2 J+1}{2} \frac{B_{\text {in }} B_{\text {out }} \Gamma^{2}}{\left(\sqrt{s}-M_{R}\right)^{2}+\Gamma^{2} / 4} R_{2}(s),
$$

onde $J$ é o spin da ressonância e $B_{\text {in }}$ e $B_{\text {out }}$ são as larguras das partículas incidente e espalhada, respectivamente. O volume do espaço de fase do momento das partículas finais é dado por $R_{2}(s)=\pi \lambda\left(s, m_{M}^{2}, m_{N}^{2}\right) / \sqrt{s} . k^{2}$ representa a função triangular $k^{2}=\lambda\left(s, m_{\pi}^{2}, m_{N}^{2}\right)$, enquanto os parâmetros $M_{R}$ e $\Gamma$ são a massa e a largura da ressonância, respectivamente.

Mais detalhes sobre os modelos acima descritos bem como testes preliminares da sua implementação para reações com fóton real podem ser encontrados em [6].

\subsection{Produção de Mésons numa Colisão Ultra-periférica}

A atual compreensão da produção de mésons vetoriais em colisões ultra-periféricas estabelece que dois processos essencialmente distintos respondem por grande parte da fotoprodução exclusiva observada experimentalmente. Um processo dito coerente e outro incoerente.

Do ponto de vista teórico, ambos os processos costumam ser definidos em termos da extensão do acoplamento do fóton com o alvo, conforme Referências [7, 8]. O processo de produção coerente se refere a um acoplamento com o núcleo alvo inteiro, o qual permanece intacto ou praticamete intacto após a emissão do méson vetorial. Praticamente intacto significa que alguns 
nêutrons podem ser emitidos embora não haja qualquer definição quanto a qual deva ser o número máximo destes. Uma sugestão de 6 já foi feita [8]. Fragmentação do núcleo também é possível $[7,8]$.

Já o processo incoerente implica no acoplamento com um único nucleon do alvo com subsequente emissão do méson. Neste caso, o núcleo normalmente se fragmenta mas a não ser por nucleons ou fragmentos pesados, outras partículas não são produzidas [8].

À luz da Teoria de Regge para interações hádron-hádron e do Modelo de Dominância Vetorial, ambos já apresentados nas Seções 2.2 e 2.3, vale reforçar, e aqui extender, que o cenário mais detalhado é aquele em que o fóton, de fato, já tendo flutuado para um par $q \bar{q}$, interage hadronicamente seja com o núcleo inteiro seja com um único nucleon, transferindo momento e então emergindo.

A caracterização experimental, contudo, não é trivial visto que ambos os processos, coerente e incoerente, são responsáveis pela população final de mésons. A distinção de processos é dependende de modelo. De fato, há o entendimento fundamental de que o processo coerente é caracterizado por baixo momento transversal do méson enquanto o incoerente deve resultar em momentos transversais mais altos. Um corte brusco em $p_{T}$ é o primeiro passo. Todavia, há contaminação entre as amostras e a estimação desta também recorre a modelo.

Toda esta situação é vista concretamente a partir da Figura 2.12, a qual reproduz resultados obtidos pela Colaboração ALICE [8].

Seis distribuições calculadas por Monte Carlo foram usadas para descrever o espectro total de $p_{T}$ :

- Produção coerente de J/ $\psi$;

- Produção incoerente de $\mathrm{J} / \psi$;

- $\mathrm{J} / \psi$ de decaimento coerente de $\psi^{\prime}$;

- $\mathrm{J} / \psi$ de decaimento incoerente de $\psi^{\prime}$; 

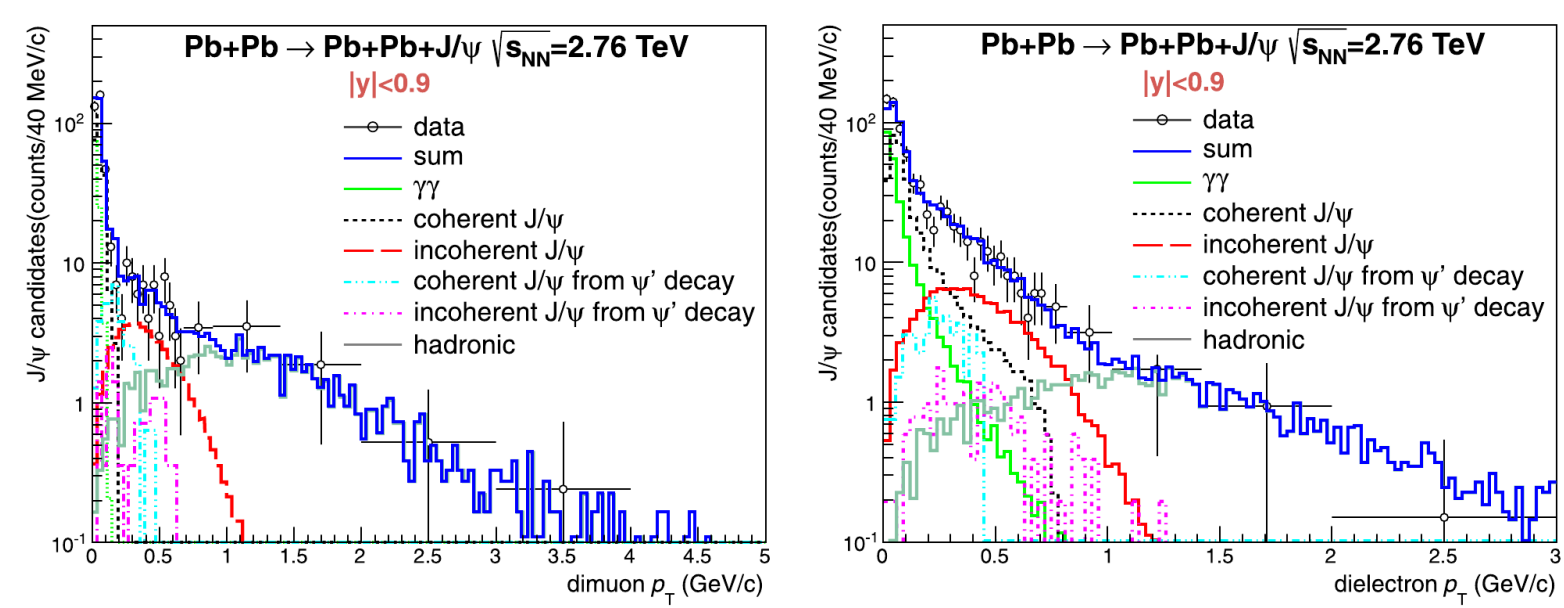

Figura 2.12: Distribuição de $p_{T}$ para di-múons (à esquerda) e di-elétrons (à direita) para a colisão ultra-periférica $\mathrm{PbPb}$ a $\sqrt{s_{N N}}=2.76 \mathrm{TeV}$ dentro do intervalo de rapidez $-0.9<y<0.9$. Dados ajustados a partir de 6 distribuições calculadas por Monte Carlo. Extraído de [8].

- Produção por processos fóton-fóton;

- Produção devida a colisão hadrônica periférica;

As cinco primeiras funções foram providas pelo modelo STARLIGHT [35]. Todos os detalhes da análise podem ser encontrados na Referência [8]. Apenas após a determinação das amostras de cada processo é que a distribuição de rapidez é calculada. Esta será mostrada no Capítulo de Resultados quando a comparação com o modelo CRISP será feita.

Vários modelos além do CRISP tem se proposto a calcular a seção de choque de fotoprodução de mésons, em particular, a distribuição de rapidez destes. As diferenças entres eles vão desde a implementação do programa até a parametrização porém dois caminhos teóricos tem sido as escolhas principais: distribuição de glúons e modelo de Glauber. As referências que substanciam as duas seguintes subseções são [17, 36, 37] por delinearem os modelos mais comuns atualmente. O próximo capítulo descreverá o Modelo CRISP em seus detalhes mais relevantes e evidenciará as diferenças entre o CRISP e os demais modelos encontrados na literatura.

Em se tratando da produção de mésons, o método de distribuição de glúons e o modelo de Glauber costumam ser apresentados no contexto da produção coerente. O mecanismo incoerente se vale das mesmas peças-chave porém será detalhado separadamente. 


\subsubsection{Produção Coerente de Mésons Vetoriais}

A seção de choque de fotoprodução exclusiva elástica do méson vetorial $V$ no alvo $H$, $(H \equiv p, A)$ é dada por

$$
\sigma^{\gamma H \rightarrow V H}(k)=\left.\frac{d \sigma^{\gamma H \rightarrow V H}}{d t}\right|_{t=0} \int d t\left|F_{H}(t)\right|^{2},
$$

onde $\left.\frac{d \sigma^{\gamma H \rightarrow V H}}{d t}\right|_{t=0}$ é a amplitude de espalhamento na direção frontal, $F_{H}(t)$ é o fator de forma do alvo e $k$ é a energia do fóton no referencial do laboratório. A informação dinâmica se encontra na amplitude de espalhamento enquanto a transferência de momento fica a cargo do fator de forma.

Seguindo a prescrição do método de distribuição de glúons, a amplitude de espalhamento para a fotoprodução elástica no núcleo é escrita como

$$
\left.\frac{d \sigma^{\gamma A \rightarrow V A}}{d t}\right|_{t=0}=\zeta_{V} \frac{16 \pi^{3} \alpha_{s}^{2} \Gamma_{e e}}{3 \alpha M_{V}^{5}}\left[x g_{A}\left(x, Q^{2}\right)\right]^{2},
$$

onde $\alpha_{s}(\alpha)$ a constante de acoplamento da força forte (eletromagnética), $\Gamma_{e e}$ a largura de decaimento leptônica, $M_{V}$ a massa do méson vetorial, $x=M_{V}^{2} / W_{\gamma p}^{2}$ é a fração do momento do nucleon carregada pelos glúons, $Q^{2}=\left(M_{V} / 2\right)^{2}$ é o momento transferido e $g_{A}\left(x, Q^{2}\right)=$ $g_{p}\left(x, Q^{2}\right) \times R_{g}^{A}\left(x, Q^{2}\right)$ é a distribuição de glúons nuclear, com $g_{p}\left(x, Q^{2}\right)$ sendo a distribuição de glúons do próton e $R_{g}^{A}\left(x, Q^{2}\right)$ a modificação na distribuição de glúons. $\zeta_{V}$ é um fator de correção estimado de forma a garantir um bom ajuste de $\sigma^{\gamma p \rightarrow V p}\left(W_{\gamma p}\right)$ com os dados experimentais de fotoprodução conforme o caso. Na Referência [17] foram usados os dados do HERA para $\mathrm{J} / \psi$

No caso de colisão $\mathrm{PbPb}$ ou $\mathrm{AuAu}$, o fator de forma nuclear tem a forma já apresentada na Equação (2.14). A seção de choque fotonuclear fica então dada por

$$
\sigma^{\gamma A \rightarrow V A}(k)=\left.\frac{d \sigma^{\gamma A \rightarrow V A}}{d t}\right|_{t=0} \int_{t_{\min }(k)}^{\infty} d t|F(t)|^{2},
$$

onde $t_{\min }(k)=\left(M_{V}^{2} / 4 k \gamma_{L}\right)^{2}$ é o momento transferido mínimo para que haja produção coerente. A determinação da seção de choque total ultra-periférica e da distribuição de rapidez segue o 
método já apresentado na Subseção 2.1.3.

Este modelo se mostrou bem sucedido na comparação com os dados recentes da Colaboração ALICE para a produção coerente do méson $J / \psi[8]$.

$\mathrm{Na}$ abordagem que segue o modelo de Glauber, a seção de choque fotonuclear permanece escrita como em (2.72) com mudança apenas no cálculo da amplitude de espalhamento frontal. Conforme Referência [36], a seção de choque $\gamma A \rightarrow V A$ tem como ponto de partida a seção de choque $\gamma p \rightarrow V p$ como parametrizada nas Equações (2.39). Para construir o caso nuclear, faz-se uso do teorema ótico e da aproximação eikonal.

Seguindo o Modelo de Dominância Vetorial,

$$
\left.\frac{d \sigma^{\gamma p \rightarrow V p}}{d t}\right|_{t=0}=\left.\frac{4 \pi \alpha}{f_{V}^{2}} \frac{d \sigma^{V p \rightarrow V p}}{d t}\right|_{t=0},
$$

sendo $f_{V}$ a constante de acoplamento fóton-méson,

$$
\frac{f_{V}^{2}}{4 \pi}=\frac{M_{V} \alpha^{2}}{3 \Gamma_{e e}} .
$$

Usando o teorema ótico, a seção de choque total méson-próton é dada por

$$
\sigma_{\text {total }}^{2}(V p)=\left.16 \pi \frac{d \sigma^{V p \rightarrow V p}}{d t}\right|_{t=0} .
$$

Este resultado entra no cálculo da seção de choque méson-núcleo seguindo Glauber,

$$
\sigma_{\text {total }}(V A)=\int d^{2} \vec{r}\left[1-\exp \left(-\sigma_{\text {total }}(V p) T_{A A}(\vec{r})\right)\right]
$$

onde $T_{A A}(\vec{r})$ é chamada função de sobreposição, que fornece o grau de intersecção das trajetórias dos íons do feixe, e dada por

$$
T_{A A}(|\vec{b}|)=\int d^{2} \vec{r} T_{A}(\vec{r}) T_{A}(\vec{r}-\vec{b})
$$

sendo $\vec{r}$ e $\vec{b}$ vetores perpendiculares à direção de propagação do feixe. A função $T_{A}(\vec{r})$, por sua 
vez é a função de espessura nuclear, calculada a partir de

$$
T_{A}(\vec{r})=\int d z \rho_{A}\left(\sqrt{|\vec{r}|^{2}+z^{2}}\right)
$$

onde $\rho_{A}$ é a densidade nuclear, calculada na Referência [36] segundo uma distribuição de Woods-Saxon.

Finalmente, o teorema ótico leva a

$$
\left.\frac{d \sigma^{\gamma A \rightarrow V A}}{d t}\right|_{t=0}=\frac{\alpha \sigma_{\text {total }}^{2}(V A)}{4 f_{V}^{2}} .
$$

\subsubsection{Produção Incoerente de Mésons Vetoriais}

Como já mencionado, a produção incoerente se vale dos mesmos ingredientes do cálculo coerente. Essa é a abordagem apresentada na Referência [37] e é o caminho seguido no modelo STARLIGHT [35].

Como se viu acima, o teorema ótico relaciona a seção de choque total à seção de choque de espalhamento frontal. Isso leva a um escalonamento de ambas quando se relaciona a seção de choque de espalhamento fóton-núcleo e fóton-próton. Usando as Equações (2.73), (2.75) e (2.79), temos,

$$
\frac{d \sigma^{\gamma A \rightarrow V A} /\left.d t\right|_{t=0}}{d \sigma^{\gamma p \rightarrow V p} /\left.d t\right|_{t=0}}=\frac{\sigma_{\text {total }}^{2}(V A)}{\sigma_{\text {total }}^{2}(V p)}=A^{2 \beta} .
$$

onde $\beta$ reflete o grau de escalonamento da seção de choque total $\gamma A$ com a seção de choque $\gamma p$ parametrizada de acordo com a equação (2.39) e que serviu que ponto de partida ao cálculo coerente. A seção de choque total incoerente é então calculada como

$$
\sigma_{\text {total }}(V A)=\left.A^{\beta} \frac{d \sigma^{\gamma p \rightarrow V p}}{d t}\right|_{t=0}
$$

Conforme a parametrização utilizada, especialmente para a seção de choque $\gamma p$, há dois limites fundamentais sejam eles $\beta=2 / 3$ e $\beta=1$, sendo que no primeiro caso a produção do méson é proporcional à contribuição da superfície nuclear enquanto no último a seção de 
choque é proporcional a todo o volume.

\subsection{Fotoprodução de Múltiplos Píons - Foto-absorção}

Considerando a fotoprodução apenas de múltiplos píons, o foca se concentra nos canais de foto-absorção dados pela reação

$$
\gamma N \rightarrow i \pi N \quad(2 \leqslant i \leqslant 8)
$$

onde $N$ se refere a próton e nêutron.

Optou-se pelo modelo proposto em [38] o qual pode ser descrito como um abordagem semiempírica que estabelece uma aproximação para o cálculo da seção de choque integral dos canais para os quais existem dados experimentais. A seção de choque integral fica então dada por

$$
\sigma(x)=\left\{\begin{array}{l}
0 \quad \text { para } E_{\gamma} \leq E_{\gamma}^{t h} \\
|F(x)|^{2} \text { para } E_{\gamma}>E_{\gamma}^{t h}
\end{array}\right.
$$

onde $x=\ln \left(E_{\gamma} / E_{\gamma}^{t h}\right)$ e $F(x)$ é a amplitude efetiva do processo. Uma vez que a fase de $F(x)$ não é determinada, esta é considerada real:

$$
F(x)=\sum_{0}^{\infty} a_{n} L_{n}^{\alpha}(x),
$$

onde $a_{n}$ são coeficientes da expansão e $L_{n}^{\alpha}(x)$ são funções ortonormais, as quais são expressas em termos dos polinômios de Laguerre generalizados $\mathcal{L}_{n}^{\alpha}(x)$ conforme

$$
L_{n}^{\alpha}(x)=e^{-x / 2} x^{\alpha / 2} \mathcal{L}_{n}^{\alpha}(x)
$$

e são normalizados de acordo com

$$
\int_{0}^{\infty} L_{n}^{\alpha}(x) L_{m}^{\alpha}(x) d x=\frac{\Gamma(n+1+\alpha)}{\Gamma(n+1)} \delta_{n m}
$$

onde $\Gamma(x)$ é a função gamma. Neste trabalho usa-se $\alpha=2$ de acordo com [38].

A reprodução das seções de choque de fotoprodução de múltiplos píons por este modelo 
pode ser comparada com os dados experimentais extraídos de [39]. Há, contudo, reações para as quais não há dados. A solução usada em [38] é também utilizada aqui. A partir dos pesos isotópicos das reações, os quais são obtidos pelo modelo estatístico de isospin, é possível conectar as seções de choque desconhecidas com aquelas para as quais há dados experimentais.

De fato, tal modelo se mostrou em bom acordo com os dados, como pode ser visto no trabalho original [38]. No entanto, o modelo lá proposto e acima descrito tem por objetivo a reprodução das seções de choque totais para as reações do tipo (2.82). Tal reação é de fato a soma de contribuições ressonantes e contribuições diretas, além de contribuições oriundas do decaimento de mésons.

Na implementação da seção de choque total $\gamma N$ para a foto-absorção, o código CRISP já considera individualmente as ressonâncias mais importantes e a foto-produção de mésons vetoriais como já descrito na última seção. Era necessária a introdução dos canais diretos de produção de múltiplos píons. O modelo semi-empírico acima descrito foi mantido para o cálculo dos canais diretos. Como a ferramenta de comparação e teste permanecem sendo as seções de choque totais, uniu-se ao modelo o cálculo das contribuições não diretas a fim de determinar a seção de choque apropriada ao canal direto que garantisse a melhor reprodução dos dados experimentais. Os resultados obtidos são apresentados no Capítulo 4.

O estado final, isto é, o quadri-momento de cada partícula produzida é determinado usando o gerador de eventos de espaço de fase do framework ROOT [30]. 


\section{Ferramentas Computacionais}

A principal ferramenta deste trabalho é o Código CRISP, o qual foi alvo de todas as implementações teóricas já descritas no Capítulo 2 e foi o originador dos resultados que serão discutidos no Capítulo 4. A seguir será apresentada inicialmente uma visão geral do programa tocando em suas principais características. Como todo este trabalho está centrado na produção de partículas que ocorre na chamada fase de cascata intranuclear, o modelo de cascata será descrito em mais detalhes na sequência.

\subsection{Código CRISP}

CRISP é um código de Monte Carlo escrito em C++ para simulação de reações nucleares [40] que utiliza um processo de cálculo baseado em duas etapas principais e mais uma que se segue apenas em caso de fissão nuclear. Primeiramente uma cascata intranuclear é simulada. Quando a partícula incidente é um próton, este interage na superfície com algum nucleon do sistema, podendo o espalhamento ser elástico ou inelástico. Fótons podem interagir mais internamente no núcleo e sua absorção dá origem a ressonâncias nucleônicas.

Partículas secundárias são produzidas, as quais possuem energias relativamente altas comparadas às dos demais nucleons no mar de Fermi e ocupam níveis de partícula única altamente energéticos [41, 42, 43]. São chamadas partículas de cascata.

As partículas secundárias geradas na colisão primária se propagam no interior do núcleo e podem interagir com outras partículas ou alcançar a superfície nuclear. Neste caso, se sua energia for superior à energia de ligação nuclear, tal partícula escapará, de outra forma será 
refletida de volta e continuará sua propagação no interior do núcleo. O número de partículas de cascata aumenta enquanto a cascata intranuclear prossegue, e a decisão de parar esta primeira etapa da reação se baseia em um critério energético, de tal forma que, não havendo qualquer partícula ligada em um estado excitado ou com energia cinética superior ao potencial nuclear, os processos de cascata são finalizados [44].

No último estágio da cascata, em que nenhuma partícula possui energia suficiente para escapar do núcleo, uma sequência de colisões distribui a energia de excitação nuclear entre todos os nucleons, num processo conhecido por termalização. As principais características do núcleo não mudam neste estágio da cascata, de modo que seu número atômico, número de massa e energia de excitação permanecem os mesmos até o fim do processo.

Um aspecto importante no código CRISP é a abordagem multi-colisional que é dada à simulação da cascata intranuclear, na qual todos os nucleons se movem simultaneamente. A sequência ordenada de colisões considera a probabilidade de interação com todas as partículas, baseando-se em suas respectivas seções de choque. Tal abordagem torna natural a verificação de aspectos dinâmicos como modificações na densidade nuclear e evolução dos níveis de ocupação.

O bloqueio de Pauli é outro mecanismo de grande relevânica que está inserido no código CRISP segundo um método preciso de verificação da disponibilidade de estados de partícula única, isto possibilitado pela abordagem multi-colisional. Sem o mecanismo de Pauli, não seria possível um critério energético para término da cascata intranuclear [45].

No código CRISP, reações podem ser iniciadas por prótons ou fótons [42, 43, 45, 46] de energias intermediárias e altas. CRISP fornece bons resultados para seções de choque de fotoabsorção, de aproximadamente $50 \mathrm{MeV}$, região em que o mecanismo de quase-dêuteron é dominante, até $3.5 \mathrm{GeV}$, onde o mecanismo de foto-hadronização é relevante, levando a efeitos de sombreamento na seção de choque [44].

A segunda etapa do processo de cálculo consiste na competição entre evaporação e fissão. O modelo de Weisskopf [47] é adotado para o cálculo das probabilidades de emissão de partículas, no qual as evaporações de nêutrons, prótons e partículas alfa são incluídas [45, 46, 48], como 
também o modelo de Dostrovsky para o cálculo dos parâmetros de densidade de níveis [49]. O modelo de Bohr-Wheeler é adotado para a fissão [50].

A distinção de uma reação nuclear nas duas etapas acima não é um fato experimental verificável, embora seja de grande valor e utilidade no estudo teórico e fenomenológico dos processos envolvidos.

Em caso de fissão do núcleo, o código CRISP pode calcular a distribuição dos fragmentos seguindo o modelo multimodo de fissão, melhor conhecido na literatura como Random Neck Rupture Model [51, 52, 53]. Conforme esse modelo, o núcleo se deforma dando origem a um estreitamento, ou pescoço, entre os futuros fragmentos. O que se segue a isso é a ruptura do sistema em algum ponto ao longo do estreitamento.

Os fragmentos formados se situam majoritariamente em três grupos, descritos por três modos de fissão, um simétrico (Superlong) e dois assimétricos (Standard I e Standard II). Cada modo é descrito por uma função gaussiana com posição centrada no fragmento de massa mais provável. Os modos assimétricos são descritos por duas gaussianas visto ser necessário contabilizar tanto a distribuição do fragmento mais pesado quanto a do fragmento leve.

Bons resultados já foram obtidos pela utilização desse método fazendo uso de três modos de fissão apenas $[54,55,56]$.

\subsubsection{Modelo de Cascata Intranuclear}

\section{Configuração inicial do alvo}

Inicialmente, o núcleo alvo é construído no centro do sistema de coordenadas segundo o modelo de Gás de Fermi. De modo que as energias de Fermi para prótons, $E_{F}^{\pi}$, e para nêutrons, $E_{F}^{v}$ são dadas por

$$
\begin{gathered}
E_{F}^{\pi}=\frac{1}{2 m_{0}}\left(3 \pi^{2}\right)^{2 / 3} \hbar^{2}\left(\frac{Z}{\Omega}\right)^{2 / 3} \mathrm{e} \\
E_{F}^{v}=\frac{1}{2 m_{0}}\left(3 \pi^{2}\right)^{2 / 3} \hbar^{2}\left(\frac{A-Z}{\Omega}\right)^{2 / 3}
\end{gathered}
$$


onde $\Omega=\frac{4}{3} \pi r_{0}^{3} A$ é o volume nuclear, considerando uma esfera de superfície bem definida, e $m_{0}$ é a massa de repouso do nucleon. Partindo da equação relativística $E^{2}=p^{2}+m_{0}^{2}$, considerando $\hbar=c=1$, escreve-se o correpondente momento de Fermi, $k_{F}$ como

$$
\begin{aligned}
& k_{F}^{\pi}=\sqrt{E_{F}^{\pi}\left(E_{F}^{\pi}+2 m_{0}\right)}, \\
& k_{F}^{v}=\sqrt{E_{F}^{v}\left(E_{F}^{v}+2 m_{0}\right)} .
\end{aligned}
$$

Cada gás de Fermi é construído independentemente nesse primeiro momento. A ocupação de cada nível é determinada pela degenerescência, considerando spin. Conhecido o número de níveis ocupados em cada gás, é calculado o tamanho da célula de momento, $d p$, a qual representa um gap no espaço dos momentos e deve ser interpretada como a incerteza no valor deste. A célula é calculada como $d p=k_{F} / N_{\text {níveis }}$. Cada componente do momento de cada nucleon recebe um múltiplo de $d p$ conforme a degenerescência podendo esta ser ainda positiva ou negativa, o que se determina de modo aleatório.

Os momentos dos prótons são então corrigidos a fim de ficarem entre $k_{\min }^{\pi}$ e $k_{F}^{v}$, sendo $k_{\min }^{\pi}=k_{F}^{v}-k_{F}^{\pi}$.

Exceto pelo raio nuclear, não há restrições à posição de cada nucleon, a qual é determinada de modo totalmente aleatório e uniforme dentro do volume do núcleo.

Os nucleons estão ligados, confinados em um poço quadrado de potencial definido por

$$
V_{0}=E_{F}^{v}+\operatorname{binding}(\sim 7 \mathrm{MeV})
$$

onde a energia de ligação e o raio nuclear são os únicos parâmetros do cálculo. O efeito da energia de ligação do potencial $V_{0}$ é levado em conta por meio da teoria da massa efetiva [57], de acordo com

$$
\sqrt{p^{2}+m_{0}^{2}}-V_{0} \equiv \sqrt{p^{2}+m^{* 2}}
$$


com $m^{*}$ sendo a massa efetiva do nucleon. Equação (3.6) ainda pode ser reescrita na forma

$$
m^{*}(p)=\sqrt{m_{0}^{2}+V_{0}^{2}-2 V_{0} \sqrt{p^{2}+m_{0}^{2}}}
$$

evidenciando que $m^{*}$ é dependente do momento do nucleon. O valor de $m^{*}$ pode ser aproximado pelo seu valor médio, de modo que

$$
\left\langle m^{*}\right\rangle=\int_{0}^{k_{F}^{v}} m^{*}(p) F(p) d p
$$

onde $F(p) d p=3\left[p^{2} /\left(k_{F}^{v}\right)^{3}\right] d p$ é a probabilidade de encontrar um nucleon com momento entre $p$ e $p+d p$. Para um núcleo de $\mathrm{Pb}$, por exemplo, encontra-se $\left\langle m^{*}\right\rangle=0.950 m_{0}$, um valor em bom acordo com a parametrização feita em [58], onde $m^{*}=(0.953 \pm 0.002) m_{0}$.

\section{Interação inicial}

O mecanismo de interação inicial dependente primordialmente da partícula incidente. $\mathrm{O}$ CRISP pode iniciar cascatas intranucleares por meio de fótons ou prótons. Em cada caso, os canais de interação apropriados são recuperados e as seções de choque de cada um são calculadas e delas dependem a escolha da interação inicial, que se dá de maneira aleatória conforme o Método de Monte Carlo.

Sendo que foto-reações são o foco deste trabalho, convém apresentar resumidamente os canais de foto-absorção presentes no CRISP. Anteriormente aos canais mais recentemente introduzidos (mésons vetoriais e multipíons), o CRISP já contava com o mecanismo de formação do quasi-dêuteron, relevante para energias do fóton entre 40 e $140 \mathrm{MeV}$. Nesse intervalo de energia, o comprimento de onda do fóton é da ordem da distância entre o par próton-nêutron. O modelo adotado para o cálculo da seção de choque [59] propõe

$$
\sigma_{q d}(\omega)=\frac{N Z}{A} \frac{2 \pi}{V} \frac{1-\gamma r_{0}}{\gamma\left(\gamma^{2}+k^{2}\right)} \sigma_{d}(\omega)
$$

onde $V$ é o volume nuclear, $k$ é o momento relativo no par próton-nêutron, $\gamma$ se relaciona ao desvio de fase de espalhamento, $\delta$, para o par próton-nêutron, por meio de $\gamma=-k \cot \delta, \sigma_{d}$ é a seção de choque de fotodesintegração do dêuteron e $r_{0}$ é o comprimento efetivo de espalha- 
mento.

Além do mecanismo de quasi-dêuteron, as ressonâncias nucleônicas de maior seção de choque também fazem parte da foto-absorção, a saber $\Delta(1232), N(1440), N(1520), N(1535)$, $N(1680)$ e $\Delta(1950)$. As ressonâncias são descritas pela fórmula de Breit-Wigner relativística

$$
\sigma=\sigma_{0} \frac{M_{0}^{2} \Gamma^{2}}{\left(W^{2}-M_{0}^{2}\right)^{2}+M_{0}^{2} \Gamma^{2}}
$$

onde $M_{0}$ é a massa central da ressonância, $\Gamma$ é sua largura, $\sigma_{0}$ é a seção de choque máxima e $W$ a energia do centro de massa do par $\gamma N$.

\section{Dinâmica da cascata}

A abordagem multicolisional permite, dentre outras coisas, calcular a cascata intranuclear numa sequência temporal de eventos em que a cada instante, que pode ser entendido como cada iteração do cálculo, o estado de cada partícula do sistema é atualizado. Essa abordagem permite uma verificação estrita do princípio de exclusão de Pauli.

O princípio de exclusão está incorporado ao modelo por meio das camadas nucleares esfericamente simétricas que formam o espaço dos momentos dos nucleons [60]. Tal abordagem implica que o número de prótons ou nêutrons com momento entre $p$ e $p+d p$ não deve exceder o número máximo permitido em cada camada ou célula de momento, calculado de acordo com o modelo do Gás de Fermi e determinado na configuração inicial do núcleo. A depleção da esfera de Fermi é levada em conta naturalmente a cada interação nucleon-nucleon, bloqueando aquelas colisões que resultam em estados finais de camadas já fechadas. No caso de um evento não bloqueado, os números de ocupação são atualizados.

Todos os possíveis processos do sistema fazem parte de uma lista construída de tal forma que o processo mais rápido ocupa a primeira posição. A lista é seguida até que um evento não seja bloqueado. Neste momento, o sistema se altera e a lista, obviamente, também é atualizada.

A abordagem multicolisional, de fato, favorece uma descrição realista da cascata uma vez que todas as partículas podem interagir entre si e a esfera de Fermi é constantemente repopulada 
dentro do volume nuclear.

Formação de ressonâncias, decaimentos e emissão de partículas são considerados neste modelo de cascata. Em particular, a emissão só é posssível se a partícula em questão alcançar a superfície nuclear. Neste ponto, uma partícula como o nêutron, sem carga, deve ter energia cinética superior ao potencial nuclear $V_{0}$ para que seja emitida. O próton por sua vez está sujeito ao potencial Coulombiano $V_{C}$, calculado como

$$
V_{C}=\frac{e^{2} Z q}{r_{0} A^{1 / 3}},
$$

onde $Z$ é a carga do núcleo, $q$ a carga do próton e $e^{2}=1.44 \mathrm{MeV} / \mathrm{fm}$. Neste caso, o CRISP também considera a probabilidade de tunelamento, calculada de acordo com a aproximação WKB. Seja $P(T)$ a probabilidade de transmissão e $T$ a energia cinética assintótica $(r \rightarrow \infty)$ da partícula a ser emitida,

$$
\begin{aligned}
& P(T)=1 \quad \text { se } \quad T>V_{C} \\
& P(T)=\exp \left[-\frac{2}{\hbar} \int_{r_{1}}^{R} \sqrt{2 m\left(V_{C}-T\right)} d r\right] \quad \text { se } \quad T<V_{C},
\end{aligned}
$$

onde $R=r_{0} A^{1 / 3}, r_{1}=\frac{e^{2} Z q}{E}$ é o ponto de retorno e $m$ é a massa da partícula.

Em caso de emissão de um nucleon, a energia de excitação nuclear é recalculada como

$$
E^{*}=E_{\gamma}-\sum_{j}\left(T_{j}+B\right)
$$

onde $E_{\gamma}$ é a energia do fóton incidente, e portanto a energia de excitação inicial, $T_{j}$ é a energia cinética assintótica do nucleon $j$ e $B \sim 7 \mathrm{MeV}$ é a energia de ligação média de um nucleon na superfície de Fermi.

\section{Critério de parada}

Em virtude da abordagem acima descrita para a cascata, outra característica importante do modelo é seu critério de parada energético. A cada passo da cascata, após a atualização de todos os observáveis físicos, é possível verificar a existência de ressonâncias ainda por decair e a existência de nucleons com energia cinética suficiente para serem emitidos do núcleo caso alcancem 
a superfície. Não havendo nenhuma das opções, a cascata intranuclear é encerrada e o núcleo é assumido termalizado, o que significa que as múltiplas colisões internas já redistribuíram de maneira suficientemente uniforme a energia de excitação.

\section{Efeito de sombreamento}

Para fótons com energia acima de $1 \mathrm{GeV}$, a função de onda já deixa de ser puramente eletromagnética e assume a forma da decomposição de Fock expressa na Equação (2.26), passando assim a ter uma componente hadrônica. O estado hadrônico é caracterizado pela energia $E_{\gamma}$, mas neste caso deve satisfazer

$$
E_{\gamma}^{2}=p^{2} c^{2}+M_{V}^{2} c^{4}
$$

Isso implica numa variação de momento entre o estado do fóton e o estado para o qual ele flutua. Dessa forma, extraindo o momento $p$ da Equação (3.14), pode-se escrever

$$
\Delta p=\frac{E_{\gamma}}{c}-p=\frac{E_{\gamma}}{c}\left[1-\sqrt{1-\frac{M_{V}^{2} c^{4}}{E_{\gamma}^{2}}}\right] .
$$

A expansão em série de Taylor do termo da raiz fornece, finalmente

$$
\Delta p=\frac{1}{2} \frac{M_{V}^{2} c^{3}}{E_{\gamma}}
$$

Pela relação de incerteza, chega-se à conclusão de que o fóton, em seu estado hadrônico, pode percorrer uma distância

$$
\Delta l=\frac{\hbar E_{\gamma}}{M_{V}^{2} c^{3}}
$$

Esse efeito de sombreamento é levado em conta com uma boa reprodução das seções de choque, a qual pode ser escrita em termos da massa do núcleo alvo na forma [6]

$$
\sigma_{V A}(A)=\sigma_{0} A^{\alpha}
$$

onde $\sigma_{0}$ é entendido como a seção de choque por nucleon e $A^{\alpha}$ deve ser interpretado como o 
número efetivo de nucleons participantes da interação $\gamma$-núcleo. Um expoente $\alpha=1$ implica em interação totalmente eletromagnética. Valores menores evidenciam a presença de sombreamento, o que pode atestado pela Figura 3.1.

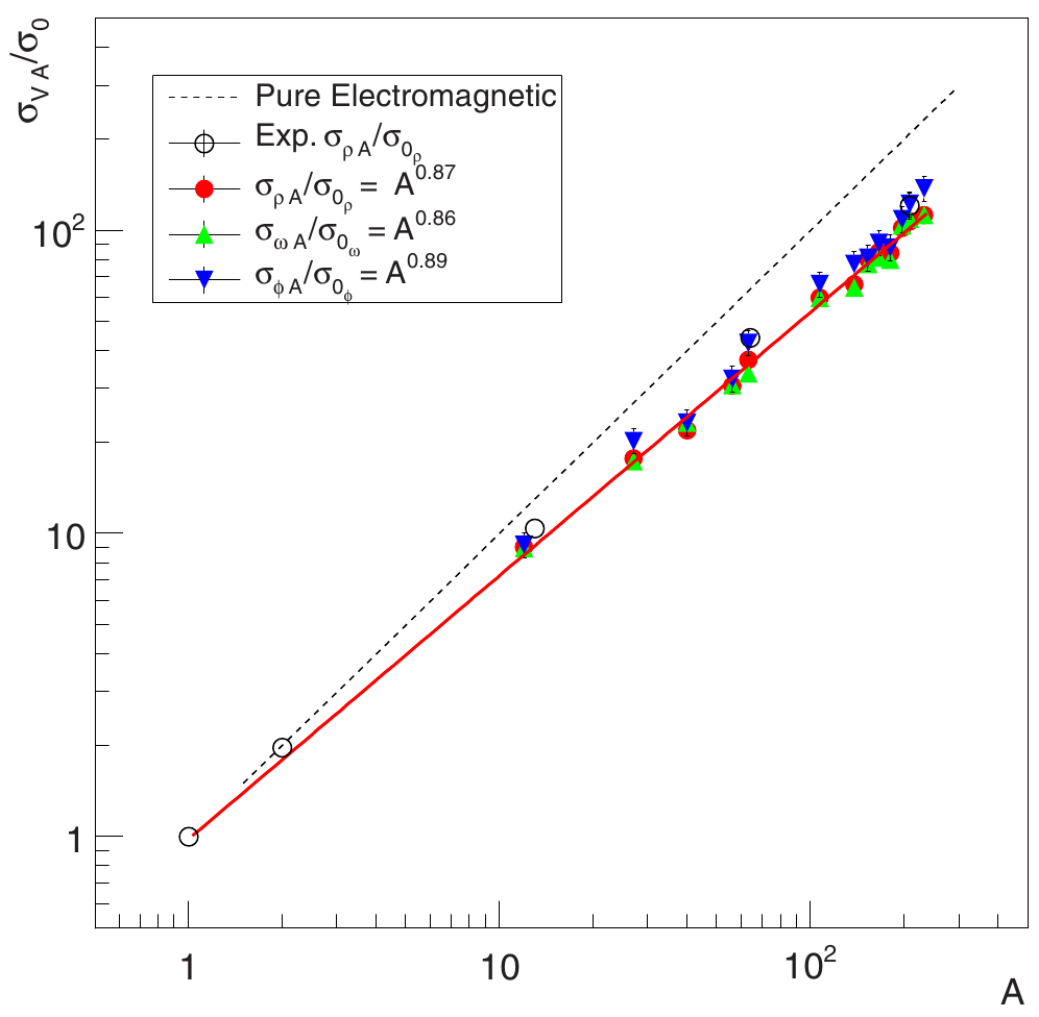

Figura 3.1: Visualização do efeito de sombreamento devido à hadronização do fóton energético. Os pontos em cores foram inteiramente calculados com o CRISP. O circulo vazio representa a seção de choque experimental de fotoprodução do méson $\rho$. Extraído de [6].

\subsection{Plataforma ROOT}

ROOT é uma plataforma orientada a objeto desenvolvida no CERN dedicada a prover soluções de cálculo e análise, em particular para a Física de Altas Energias. A plataforma está escrita em linguagem $\mathrm{C}++$ e suas ferramentas estão implementadas de maneira modular ou em classes. A grande maioria dessas classes fornece funcionalidades de natureza fundamental, de modo a não restringir seu uso apenas à Física. Assim, o ROOT é facilmente integrado ao cálculo e análise em qualquer outra área, seja acadêmica ou tecnológica.

Uma ferramenta integra a plataforma ROOT e é utilizada no código CRISP. Trata-se da classe TGenPhaseSpace, um gerador de eventos de estado final que calcula os quadri-momentos 
finais das partículas dentro do espaço de fase acessível do sistema, usando para tanto o método de Raubold-Lynch [61].

O ROOT v5.34 foi utlizado para a realização deste trabalho.

\subsection{Outras Ferramentas}

A ferramenta openmpi [62] é uma interface para passagem de mensagens entre processadores que viabiliza a computação paralela, a qual foi necessária à realização deste trabalho dado o custo computacional de realizar um grande número de cascatas intranucleares.

A Gnu Scientific Libray (GSL) [63] também é utilizada pelo CRISP internamente no cálculo de funções e polinôminos ainda não constantes da plataforma ROOT. Os polinômios associados de Laguerre, necessários ao modelo de fotoprodução de múltiplos píons foram calculados a partir dos algoritmos do GSL. 


\section{$4 \quad$ Resultados e Discussão}

\subsection{Fotoprodução de Múltiplos Píons - Foto-absorção}

O cálculo das seções de choque de fotoprodução de píons por meio do modelo apresentado na seção 2.5 pode ser comparado com os dados experimentais extraídos de [39]. A qualidade da reprodução é considerada boa em sua maior parte e pode ser vista nas Figuras 4.1, 4.2 e 4.3.

Algumas dessas reações, no entanto estão entre aquelas que, como já mencionado, se originam de múltiplas contribuições ressonantes e diretas à seção de choque total.

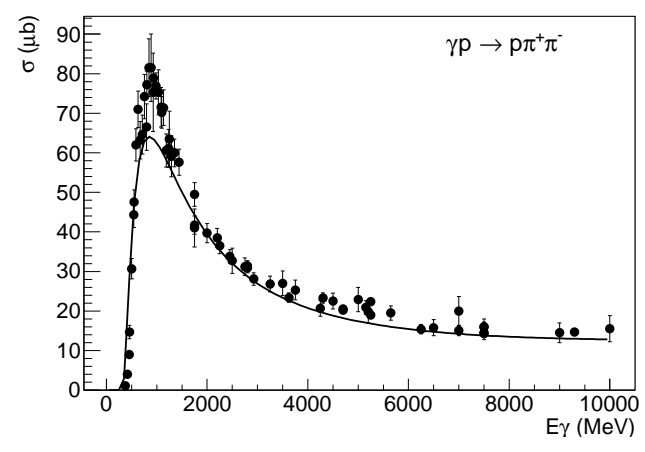

(a)

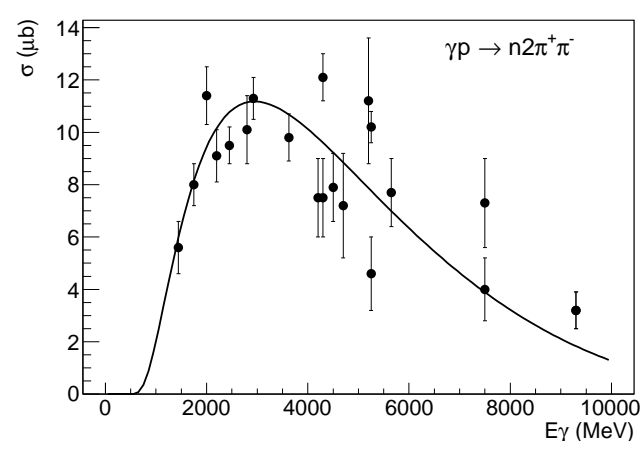

(c)

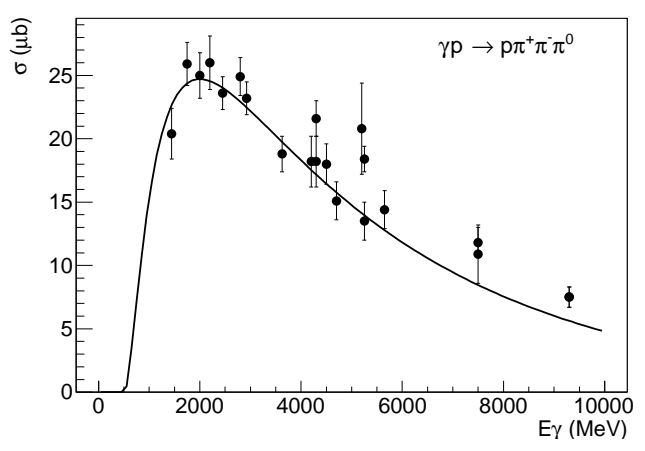

(b)

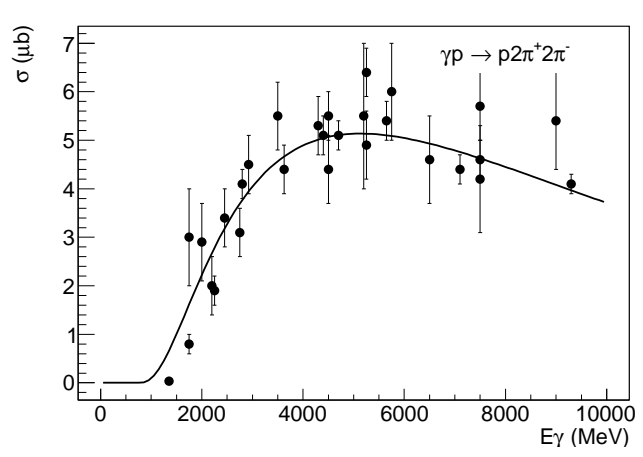

(d)

Figura 4.1: Seções de choque de fotoprodução de múltiplos píons (Parte 1). Dados experimentais obtidos de [39] 


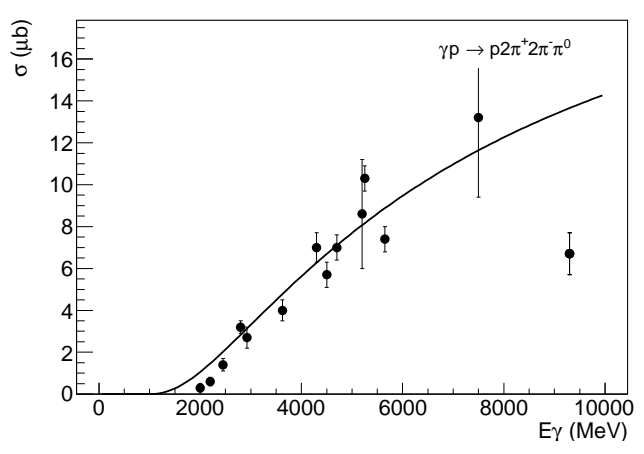

(a)

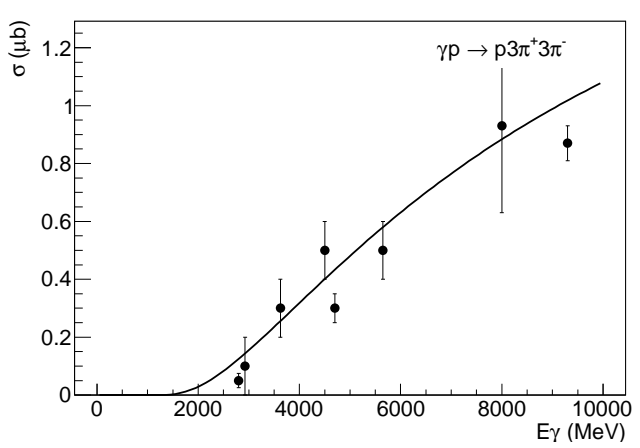

(c)

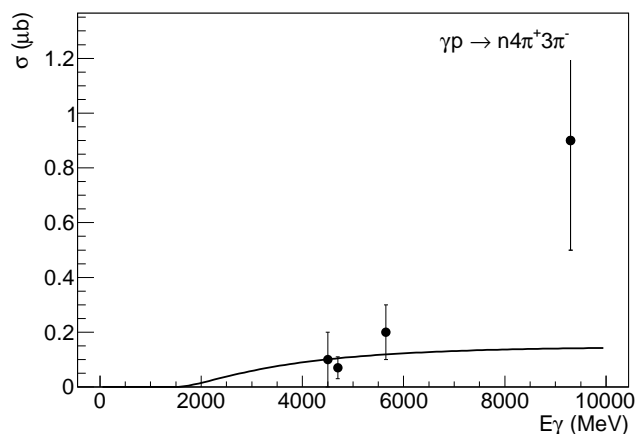

(e)

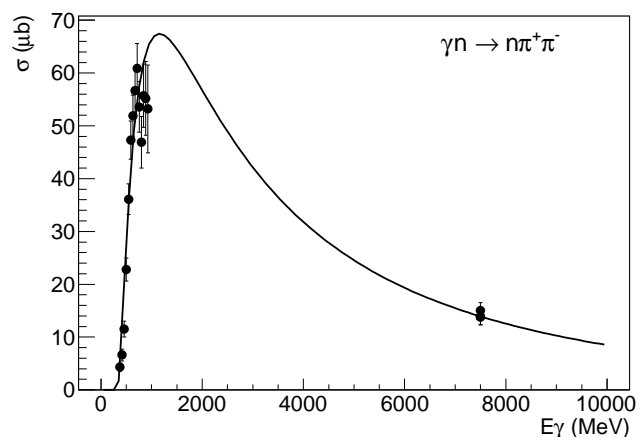

(g)

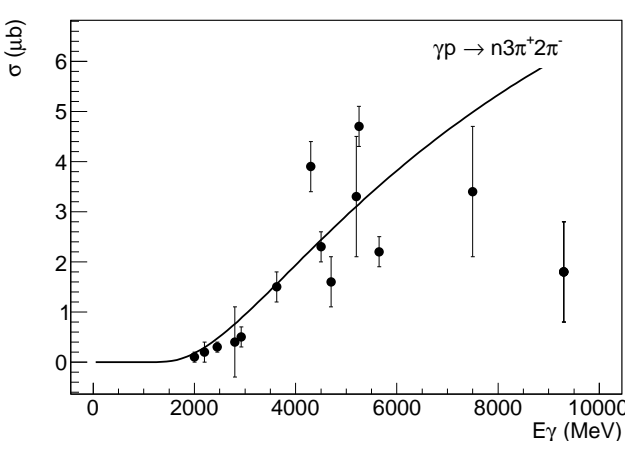

(b)

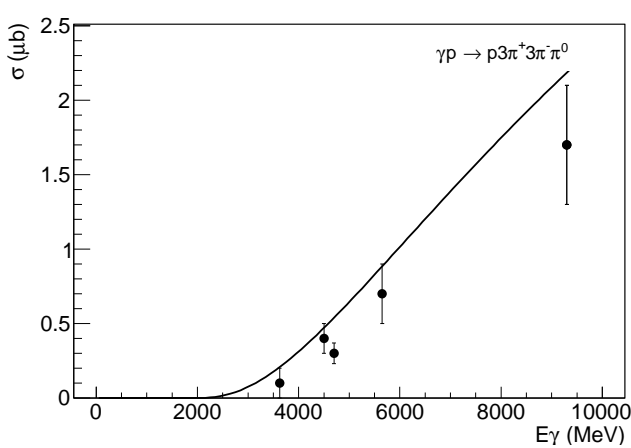

(d)

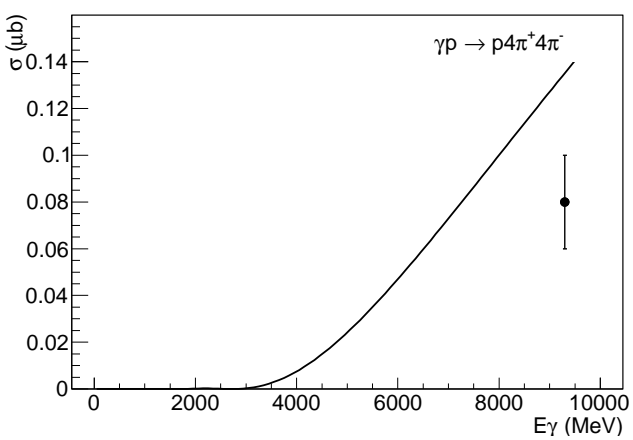

(f)

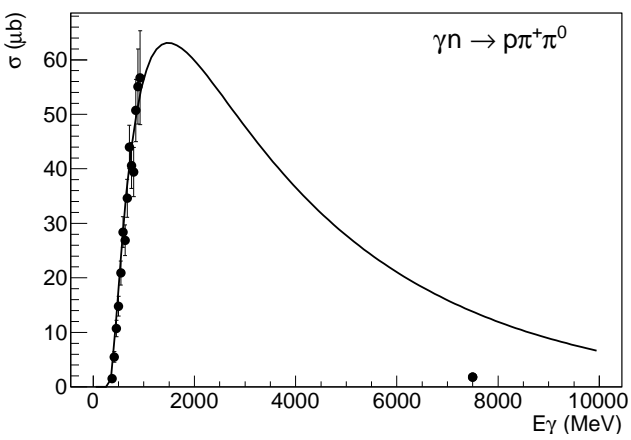

(h)

Figura 4.2: Seções de choque de fotoprodução de múltiplos píons (Parte 2). Dados experimentais obtidos de [39] 


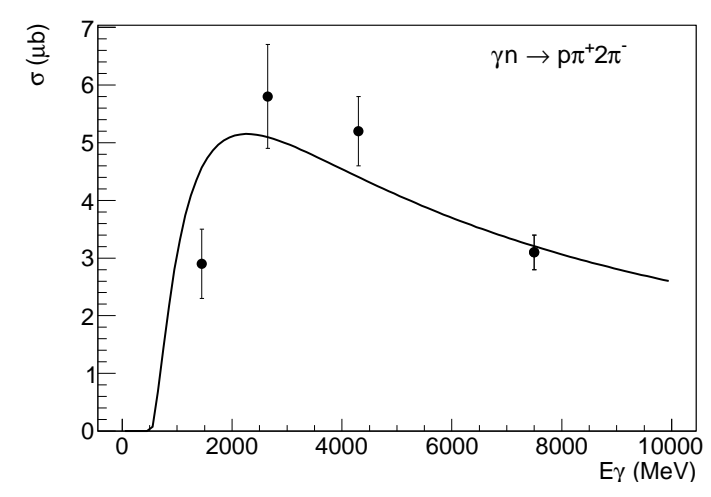

(a)

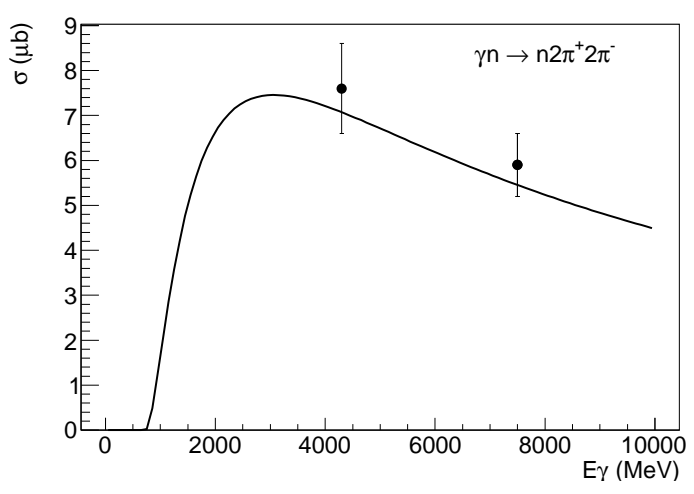

(b)

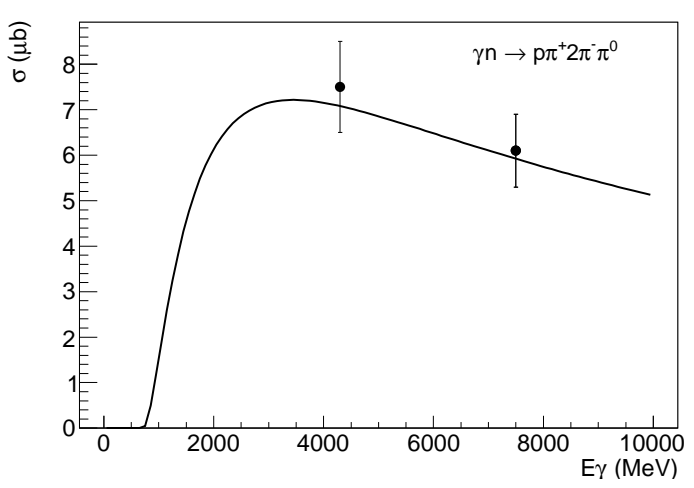

(c)

Figura 4.3: Seções de choque de fotoprodução de múltiplos píons (Parte 3). Dados experimentais obtidos de [39]

\section{Contribuições ressonantes à fotoprodução de múltiplos píons}

Várias ressonâncias nucleônicas e mésons vetoriais podem decair em um ou mais píons e contribuem, portanto, para a seção de choque total de fotoprodução. Ressonâncias nucleônicas são particularmente importantes na fotoprodução de 2 píons enquanto mésons vetoriais, ao decaírem, podem contribuir para a produção de 2 a 5 píons.

As contribuições que foram contabilizadas estão listadas a seguir para cada canal de fotoprodução e foram obtidas do Particle Data Group [15]. Os processos marcados com (*) não existiam previamente no código CRISP e foram introduzidos neste trabalho. Isto no que diz respeito ao decaimento das ressonâncias mais pesadas do que a $\Delta(1232)$. Os decaimentos mais importantes desta ressonância, bem como os decaimentos dos mésons vetoriais, já haviam sido inseridos no código. 
- $\gamma p \longrightarrow \pi^{+} \pi^{-} p$

$$
\begin{aligned}
& \Delta(1950) \longrightarrow \Delta^{0} \pi^{+} \longrightarrow \pi^{+} \pi^{-} p(*) \\
& \Delta(1950) \longrightarrow \Delta^{++} \pi^{-} \longrightarrow \pi^{+} \pi^{-} p(*) \\
& \Delta(1950) \longrightarrow \rho^{0} p \longrightarrow \pi^{+} \pi^{-} p(*) \\
& N(1440) \longrightarrow \Delta^{++} \pi^{-} \longrightarrow \pi^{+} \pi^{-} p \\
& N(1440) \longrightarrow \Delta^{0} \pi^{+} \longrightarrow \pi^{+} \pi^{-} p \\
& N(1440) \longrightarrow \pi^{+} \pi^{-} p(*) \\
& N(1520) \longrightarrow \Delta^{++} \pi^{-} \longrightarrow \pi^{+} \pi^{-} p \\
& N(1520) \longrightarrow \Delta^{0} \pi^{+} \longrightarrow \pi^{+} \pi^{-} p \\
& N(1520) \longrightarrow \pi^{+} \pi^{-} p(*) \\
& N(1520) \longrightarrow \rho^{0} p \longrightarrow \pi^{+} \pi^{-} p(*) \\
& N(1535) \longrightarrow \Delta^{++} \pi^{-} \longrightarrow \pi^{+} \pi^{-} p \\
& N(1535) \longrightarrow \Delta^{0} \pi^{+} \longrightarrow \pi^{+} \pi^{-} p \\
& N(1680) \longrightarrow \Delta^{++} \pi^{-} \longrightarrow \pi^{+} \pi^{-} p \\
& N(1680) \longrightarrow \Delta^{0} \pi^{+} \longrightarrow \pi^{+} \pi^{-} p \\
& N(1680) \longrightarrow \pi^{+} \pi^{-} p(*) \\
& N(1680) \longrightarrow \rho^{0} p \longrightarrow \pi^{+} \pi^{-} p \\
& \rho^{0} p \longrightarrow \pi^{+} \pi^{-} p
\end{aligned}
$$$$
\text { - } \gamma p \longrightarrow \pi^{0} \pi^{0} p
$$

$$
\Delta(1950) \longrightarrow \Delta^{+} \pi^{0} \longrightarrow \pi^{0} \pi^{0} p\left({ }^{*}\right)
$$




$$
\begin{aligned}
& N(1440) \longrightarrow \Delta^{+} \pi^{0} \longrightarrow \pi^{0} \pi^{0} p \\
& N(1440) \longrightarrow \pi^{0} \pi^{0} p\left(^{*}\right) \\
& N(1520) \longrightarrow \Delta^{+} \pi^{0} \longrightarrow \pi^{0} \pi^{0} p \\
& N(1520) \longrightarrow \pi^{0} \pi^{0} p\left(^{*}\right) \\
& N(1535) \longrightarrow \Delta^{+} \pi^{0} \longrightarrow \pi^{0} \pi^{0} p \\
& N(1680) \longrightarrow \Delta^{+} \pi^{0} \longrightarrow \pi^{0} \pi^{0} p \\
& N(1680) \longrightarrow \pi^{0} \pi^{0} p(*)
\end{aligned}
$$

- $\gamma p \longrightarrow \pi^{+} \pi^{0} n$

$$
\begin{aligned}
& \Delta(1950) \longrightarrow \Delta^{+} \pi^{0} \longrightarrow \pi^{+} \pi^{0} n\left(^{*}\right) \\
& \Delta(1950) \longrightarrow \Delta^{0} \pi^{+} \longrightarrow \pi^{+} \pi^{0} n\left(^{*}\right) \\
& N(1440) \longrightarrow \Delta^{+} \pi^{0} \longrightarrow \pi^{+} \pi^{0} n \\
& N(1440) \longrightarrow \Delta^{0} \pi^{+} \longrightarrow \pi^{+} \pi^{0} n \\
& N(1440) \longrightarrow \pi^{+} \pi^{0} n\left(^{*}\right) \\
& N(1520) \longrightarrow \Delta^{+} \pi^{0} \longrightarrow \pi^{+} \pi^{0} n \\
& N(1520) \longrightarrow \Delta^{0} \pi^{+} \longrightarrow \pi^{+} \pi^{0} n \\
& N(1520) \longrightarrow \pi^{+} \pi^{0} n(*) \\
& N(1520) \longrightarrow \rho^{+} n \longrightarrow \pi^{+} \pi^{0} n(*) \\
& N(1535) \longrightarrow \Delta^{0} \pi^{+} \longrightarrow \pi^{+} \pi^{0} n
\end{aligned}
$$




$$
\begin{aligned}
& N(1680) \longrightarrow \Delta^{+} \pi^{0} \longrightarrow \pi^{+} \pi^{0} n \\
& N(1680) \longrightarrow \Delta^{0} \pi^{+} \longrightarrow \pi^{+} \pi^{0} n \\
& N(1680) \longrightarrow \pi^{+} \pi^{0} n\left(^{*}\right) \\
& N(1680) \longrightarrow \rho^{+} n \longrightarrow \pi^{+} \pi^{0} n \\
& \rho^{+} n \longrightarrow \pi^{+} \pi^{0} n
\end{aligned}
$$

- $\gamma n \longrightarrow \pi^{+} \pi^{-} n$

$$
\begin{aligned}
& \Delta(1950) \longrightarrow \Delta^{+} \pi^{-} \longrightarrow \pi^{+} \pi^{-} n\left(^{*}\right) \\
& \Delta(1950) \longrightarrow \Delta^{-} \pi^{+} \longrightarrow \pi^{+} \pi^{-} n\left(^{*}\right) \\
& \Delta(1950) \longrightarrow \rho^{0} n \longrightarrow \pi^{+} \pi^{-} n\left(^{*}\right) \\
& N(1440) \longrightarrow \Delta^{+} \pi^{-} \longrightarrow \pi^{+} \pi^{-} n \\
& N(1440) \longrightarrow \Delta^{-} \pi^{+} \longrightarrow \pi^{+} \pi^{-} n \\
& N(1440) \longrightarrow \pi^{+} \pi^{-} n(*) \\
& N(1520) \longrightarrow \Delta^{+} \pi^{-} \longrightarrow \pi^{+} \pi^{-} n \\
& N(1520) \longrightarrow \Delta^{-} \pi^{+} \longrightarrow \pi^{+} \pi^{-} n \\
& N(1520) \longrightarrow \pi^{+} \pi^{-} n\left(^{*}\right) \\
& N(1520) \longrightarrow \rho^{0} n \longrightarrow \Delta^{+} \pi^{-} \longrightarrow \pi^{+} \pi^{-} n \\
& N(1680) \longrightarrow \Delta^{-} \pi^{+} \longrightarrow \pi^{+} \pi^{-} n \\
& N(1535) \longrightarrow \Delta^{+} \pi^{-} \longrightarrow \pi^{+} \pi^{-} n \\
& N(1535) \longrightarrow \Delta^{-} \pi^{+} \longrightarrow \pi^{+} \pi^{-} n \\
& N(1680) \longrightarrow \pi^{*} n
\end{aligned}
$$




$$
\begin{aligned}
& N(1680) \longrightarrow \pi^{+} \pi^{-} n(*) \\
& N(1680) \longrightarrow \rho^{0} n \longrightarrow \pi^{+} \pi^{-} n \\
& \rho^{0} n \longrightarrow \pi^{+} \pi^{-} n \\
& \text { - } \gamma n \longrightarrow \pi^{-} \pi^{0} p \\
& \Delta(1950) \longrightarrow \Delta^{+} \pi^{-} \longrightarrow \pi^{-} \pi^{0} p\left({ }^{*}\right) \\
& \Delta(1950) \longrightarrow \Delta^{0} \pi^{0} \longrightarrow \pi^{-} \pi^{0} p(*) \\
& \Delta(1950) \longrightarrow \rho^{-} p \longrightarrow \pi^{-} \pi^{0} p(*) \\
& N(1440) \longrightarrow \Delta^{+} \pi^{-} \longrightarrow \pi^{-} \pi^{0} p \\
& N(1440) \longrightarrow \Delta^{0} \pi^{0} \longrightarrow \pi^{-} \pi^{0} p \\
& N(1440) \longrightarrow \pi^{-} \pi^{0} p(*) \\
& N(1520) \longrightarrow \Delta^{+} \pi^{-} \longrightarrow \pi^{-} \pi^{0} p \\
& N(1520) \longrightarrow \Delta^{0} \pi^{0} \longrightarrow \pi^{-} \pi^{0} p \\
& N(1520) \longrightarrow \pi^{-} \pi^{0} p(*) \\
& N(1520) \longrightarrow \rho^{-} p \longrightarrow \pi^{-} \pi^{0} p(*) \\
& N(1535) \longrightarrow \Delta^{+} \pi^{-} \longrightarrow \pi^{-} \pi^{0} p \\
& N(1535) \longrightarrow \Delta^{0} \pi^{0} \longrightarrow \pi^{-} \pi^{0} p \\
& N(1680) \longrightarrow \Delta^{+} \pi^{-} \longrightarrow \pi^{-} \pi^{0} p \\
& N(1680) \longrightarrow \Delta^{0} \pi^{0} \longrightarrow \pi^{-} \pi^{0} p \\
& N(1680) \longrightarrow \pi^{-} \pi^{0} p(*) \\
& N(1680) \longrightarrow \rho^{-} p \longrightarrow \pi^{-} \pi^{0} p
\end{aligned}
$$




$$
\rho^{-} p \longrightarrow \pi^{-} \pi^{0} p
$$

- $\gamma n \longrightarrow \pi^{0} \pi^{0} n$

$$
\begin{aligned}
& \Delta(1950) \longrightarrow \Delta^{0} \pi^{0} \longrightarrow \pi^{0} \pi^{0} n\left(^{*}\right) \\
& N(1440) \longrightarrow \Delta^{0} \pi^{0} \longrightarrow \pi^{0} \pi^{0} n \\
& N(1440) \longrightarrow \pi^{0} \pi^{0} n\left(^{*}\right) \\
& N(1520) \longrightarrow \Delta^{0} \pi^{0} \longrightarrow \pi^{0} \pi^{0} n \\
& N(1520) \longrightarrow \pi^{0} \pi^{0} n\left(^{*}\right) \\
& N(1535) \longrightarrow \Delta^{0} \pi^{0} \longrightarrow \pi^{0} \pi^{0} n \\
& N(1680) \longrightarrow \Delta^{0} \pi^{0} \longrightarrow \pi^{0} \pi^{0} n \\
& N(1680) \longrightarrow \pi^{0} \pi^{0} n(*)
\end{aligned}
$$

- $\gamma N \longrightarrow \pi^{+} \pi^{-} \pi^{0} N$

$$
\omega N \longrightarrow \pi^{+} \pi^{-} \pi^{0} N
$$

- $\gamma N \longrightarrow \pi^{+} \pi^{-} \pi^{0} \pi^{0} N$

$$
\phi N \longrightarrow K^{+} K^{-} N \longrightarrow \pi^{+} \pi^{-} \pi^{0} \pi^{0} N
$$

- $\gamma N \longrightarrow \pi^{0} \pi^{0} \pi^{0} \pi^{0} \pi^{0} N$

$$
\phi N \longrightarrow K_{L O} K_{S O} N \longrightarrow \pi^{0} \pi^{0} \pi^{0} \pi^{0} \pi^{0} N
$$

- $\gamma N \longrightarrow \pi^{+} \pi^{-} \pi^{0} \pi^{0} \pi^{0} N$ 
$\phi N \longrightarrow K_{L O} K_{S O} N \longrightarrow \pi^{+} \pi^{-} \pi^{0} \pi^{0} \pi^{0} N$

- $\gamma N \longrightarrow \pi^{+} \pi^{+} \pi^{-} \pi^{-} \pi^{0} N$

$$
\phi N \longrightarrow K_{L O} K_{S O} N \longrightarrow \pi^{+} \pi^{+} \pi^{-} \pi^{-} \pi^{0} N
$$

A implementação dessas contribuições ressonantes e de mésons vetoriais foi feita por meio de uma função nomeada de parte ressonante. Cada canal de produção de píons tem a sua.

A fim de não inserir no código contribuições duplicadas de produção de píons (uma vez pelas contribuições individuais acima e outra pelo modelo proposto que já fornece a seção de choque total de cada canal) era necessário então subtrair do modelo a parte ressonante e introduzir como canal de interação fóton-nucleon apenas o que fosse não-ressonante. Essa foi a ideia inicial.

Em um segundo momento, observou-se como mais realista fisicamente obter a parte de produção direta de píons não por subtração. Fisicamente, a seção de choque de produção direta (não-ressonante) deve ser uma curva suave. Uma subtração garante que a seção de choque total proposta pelo modelo nunca seja ultrapassada, mas o resultado da subtração produz várias estruturas e artefatos sem significado físico. Um exemplo disso está apresentado na Figura 4.4.

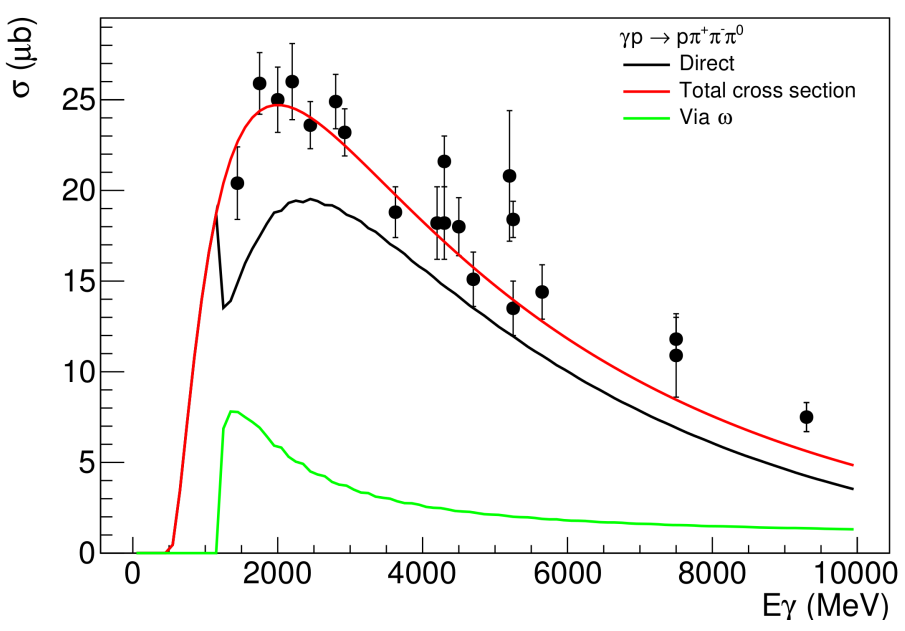

Figura 4.4: Exemplo do procedimento de subtração de uma contribuição por méson $\omega$ na fotoprodução de píons na reação $\gamma p \longrightarrow p \pi^{+} \pi^{-} \pi^{0}$. Dados experimentais [39]. 
O pico apresentado pela parte direta em torno de $1000 \mathrm{MeV}$ não tem sentido físico.

Além desse fato, a seção de choque total proposta pelo modelo não era necessariamente a melhor em todos os casos. Optou-se assim por buscar uma forma suave para os mecanismos diretos de produção porém ainda se atendo aos polinômios de Laguerre da forma como descrito pelas Equações (2.83) a (2.86) como ferramenta. Isso foi feito de forma que todas as contribuições somadas, direta, ressonante e de mésons vetoriais ainda fornecessem uma boa seção de choque total.

O código CRISP combina os métodos de Monte Carlo e de Dinâmica Molecular Quântica para calcular vários aspectos dos processos de reações nucleares induzidos a diferentes energias por diferentes partículas. O método é semi-clássico, utilizando as trajetórias das partículas envolvidas na reação. Isto é válido quando o comprimento de onda dessas partículas é consideravelmente menor do que a distância entre elas. Nesta situação, a seção de choque total pode ser considerada como a soma incoerente das diversas seções de choque envolvidas.

De fato, no cálculo da amplitude de transição entre dois estados, a probabilidade de transição $\left|K\left(x_{i}\left(t_{i}\right), x_{f}\left(t_{f}\right)\right)\right|^{2}$ entre o estado inicial e o final é dada pela contribuição de cada possível caminho $C$ entre os mesmos estados inicial e final, calculando-se para isso a amplitude $K$ como

$$
K\left(x_{i}\left(t_{i}\right), x_{f}\left(t_{f}\right)\right)=\sum_{\text {caminho C }} \exp \left[\frac{\mathrm{i}}{\hbar} S(x(t), \dot{x}(t))\right] .
$$

A ação $S$ relacionada a um dos possíveis caminhos entre o estado inicial e o final se torna muito diferente da ação relacionada aos demais caminhos, principalmente devido ao potencial diferente a que cada partícula é sujeita. Assim, não há termos cruzados apreciáveis no cálculo da amplitude total neste caso.

\section{Determinação das contribuições diretas na fotoprodução de múltiplos píons}

Para esse cálculo, buscou-se o melhor ajuste com o menor número de polinômios possível. Notou-se, inclusive, que para o fim desejado, não há contribuições significativas ou úteis à forma da função a partir do sétimo polinômio. Também se verificou que o ajuste poderia ser 
melhor se fosse permitida à parte direta da fotoprodução ter um limiar de energia mínima do fóton superior ao limiar do processo global.

A Tabela 4.1 mostra os valores obtidos para os parâmetros $a_{n}$ da Equação (2.84) no ajuste da parte direta da fotoprodução, bem como seu limiar e o limiar global do processo.

Tabela 4.1: Parâmetros do ajuste da parte direta (não-ressonante) da fotoprodução de múltiplos píons. Energias em MeV. Incertezas informadas entre parêntesis.

\begin{tabular}{|c|c|c|c|c|c|c|c|c|}
\hline \multirow[t]{2}{*}{ Canal $\gamma N$} & \multirow{2}{*}{$\begin{array}{c}\text { Limiar } \\
\text { Global } E_{\gamma}\end{array}$} & \multirow{2}{*}{$\begin{array}{c}\text { Limiar } \\
E_{\gamma}\end{array}$} & \multicolumn{6}{|c|}{ Parâmetros do ajuste } \\
\hline & & & $a_{0}$ & $a_{1}$ & $a_{2}$ & $a_{3}$ & $a_{4}$ & $a_{5}$ \\
\hline$\gamma n \rightarrow n \pi^{+} \pi^{-}$ & 322 & 420 & $0.11143(3)$ & $0.171525(5)$ & $-0.082725(5)$ & $0.044725(5)$ & $-0.002(1)$ & $0.02(1)$ \\
\hline$\gamma n \rightarrow p \pi^{-} \pi^{0}$ & 313 & 500 & $0.004743(5)$ & $0.110925(5)$ & $-0.0082725(5)$ & $0.052725(5)$ & $-0.05(1)$ & $0.05(1)$ \\
\hline$\gamma p \rightarrow n \pi^{+} \pi^{0}$ & 321 & 470 & $0.095179(5)$ & $-0.02418(3)$ & $0.11976(3)$ & $0.0013(2)$ & $-0.064(1)$ & $0.054(1)$ \\
\hline$\gamma p \rightarrow p \pi^{+} \pi^{-}$ & 321 & 480 & $0.165179(5)$ & $-0.03118(3)$ & $0.12976(3)$ & $0.0013(2)$ & $-0.04(1)$ & $0.036(1)$ \\
\hline$\gamma p \rightarrow p \pi^{+} \pi^{-} \pi^{0}$ & 506 & 556 & $0.03379(3)$ & $0.14304(3)$ & $-0.0457(2)$ & & & \\
\hline$\gamma p \rightarrow p 2 \pi^{+} 2 \pi^{-} \pi^{0}$ & 952 & 952 & $0.20568(3)$ & $-0.06299(3)$ & & & & \\
\hline
\end{tabular}

A Figura 4.5 mostra o resultado do ajuste feito. Embora a seção de choque total seja a que agora apresenta descontinuidades, isso ainda foi considerado preferível.

Note-se que a lista, apresentada na seção 4.1, de canais de fotoprodução que admitem múltiplas contribuições à seção de choque contém mais do que 6 reações. As diversas contribuições à seção de choque são conhecidas fisicamente mas as únicas reações para as quais há dados experimentais são aquelas apresentadas na Figura 4.5.

Os demais resultados para os quais há dados experimentais, mas que não requerem análise de canais diretos e indiretos permanecem obedecendo a proposta da Referência [38].

Uma última consideração a ser feita sobre as seções de choque de fotoprodução de múltiplos píons diz respeito ao fato de não haver modelo para o cálculo da seção de choque na interação $\gamma n$ a partir de 5 píons nem dados experimentais (a não ser para o valor total da seção de choque, mas não como função da energia do fóton). Foi decidido então, que os canais $\gamma n$ ausentes teriam a mesma seção de choque do canal $\gamma p$ equivalente, levando-se em conta, é claro, as diferenças de carga no momento da criação das partículas durante a execução do Monte Carlo. Embora a seção de choque de fotoprodução no próton deva ser maior, como se verifica para os casos medidos, as seções de choque com mais píons no estado final são menores. De modo que o erro cometido deverá ser pequeno quando comparado à seção de choque total de fotoprodução no 


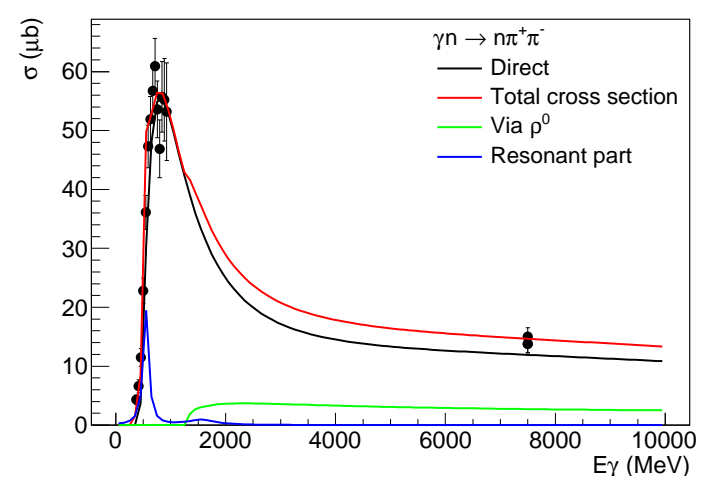

(a) $\gamma n \longrightarrow n \pi^{+} \pi^{-}$

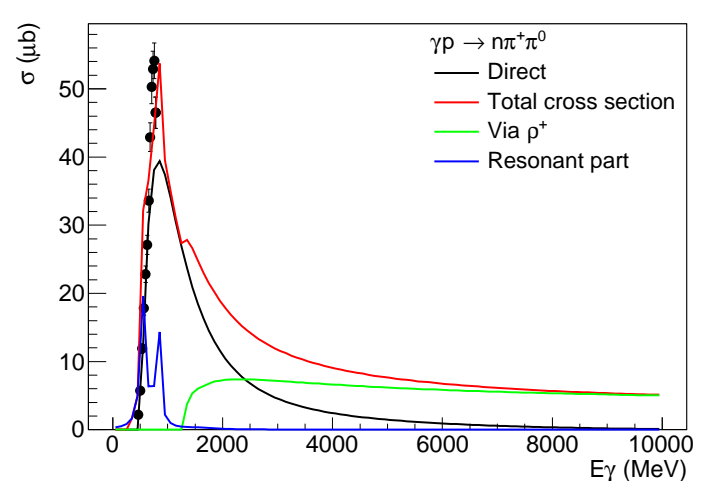

(c) $\gamma p \longrightarrow n \pi^{+} \pi^{0}$

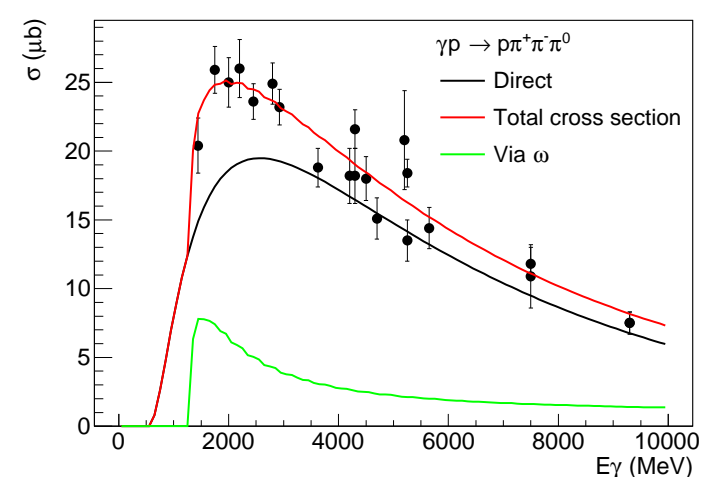

(e) $\gamma p \longrightarrow p \pi^{+} \pi^{-} \pi^{0}$

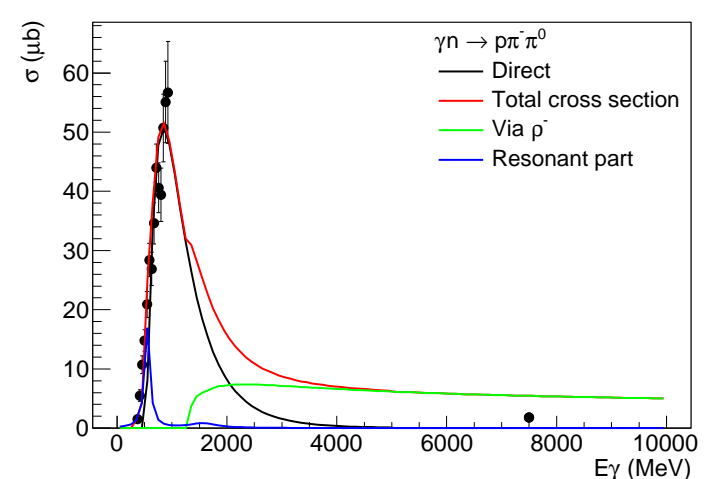

(b) $\gamma n \longrightarrow p \pi^{-} \pi^{0}$

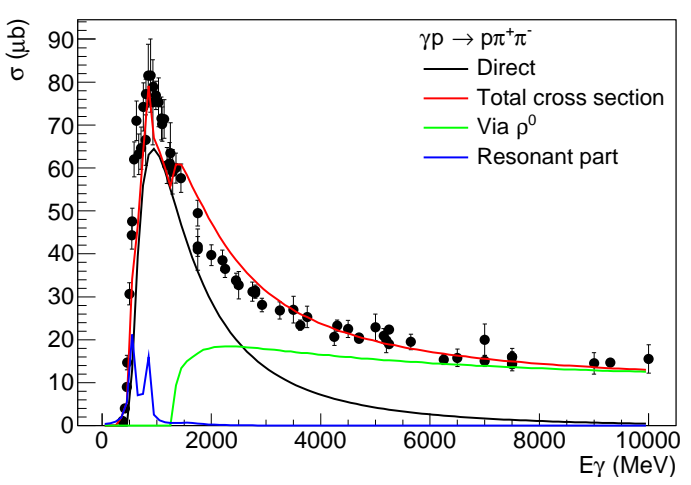

(d) $\gamma p \longrightarrow p \pi^{+} \pi^{-}$

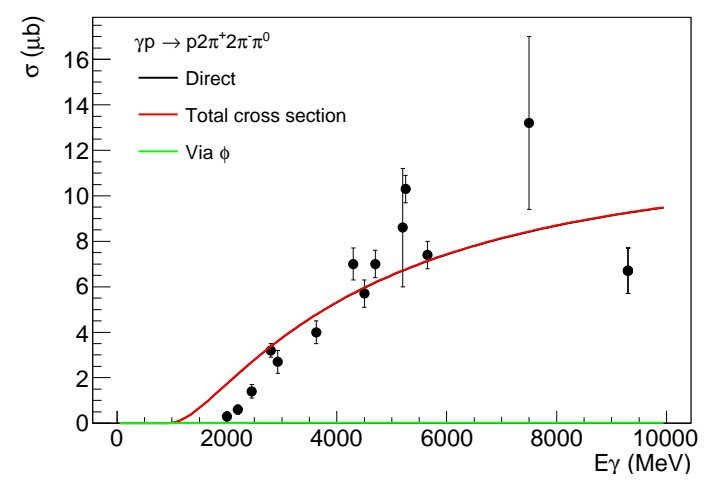

(f) $\gamma p \longrightarrow p 2 \pi^{+} 2 \pi^{-} \pi^{0}$

Figura 4.5: Seções de choque de fotoprodução de mútiplos píons. Dados experimentais [39]. Na Figura (b) os dados são de [64].

nêutron. A alternativa significaria deixar ausentes diversos canais de produção que reduziriam a população de píons de uma quantidade apreciável. De fato, o cálculo da fotoprodução nuclear de píons será o indicador mais confiável do quão boa é esta estratégia.

Considerando que foram inseridos canais de fotoprodução de 2 a 8 píons, cada um com sua mutiplicidade devida às cargas dos produtos finais, e isto para interação com próton e nêutron, 
um total de 84 canais de fotoprodução de píons foram adicionados ao modelo.

\subsection{Fotoprodução do méson $\mathrm{J} / \psi$}

Para os resultados a seguir, referentes ao cálculo da seção de choque de fotoprodução do méson $\mathrm{J} / \psi$ foi seguido o formalismo descrito na subseção 2.1.3. A consistência do modelo empregado neste trabalho pode ser atestada por meio da Figura 4.6, onde é mostrada a seção de choque de fotoprodução do méson no próton como calculada pelo modelo em comparação com medidas da Colaboração ALICE para colisão ultra-periférica pPb [65].

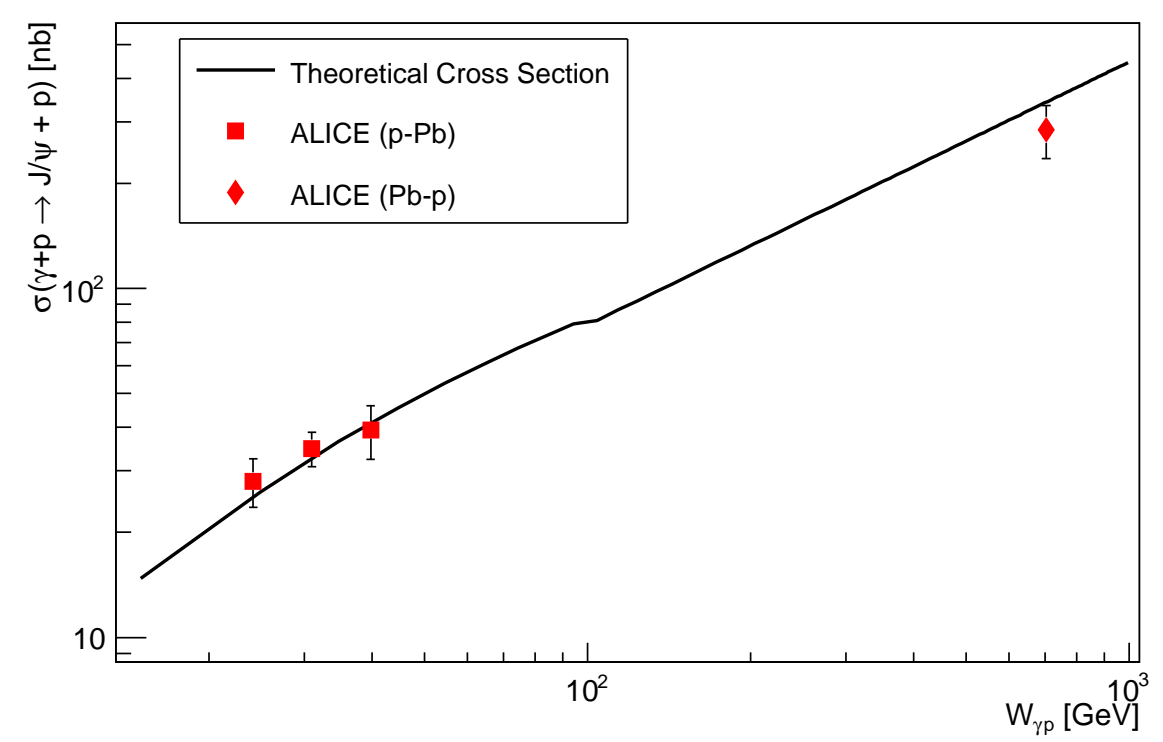

Figura 4.6: Seção de choque de produção do méson $\mathrm{J} / \psi$ no próton em função da energia no centro de massa do par $\gamma p$. Dados experimentais obtidos pela Colaboração ALICE por meio de medidas em colisão ultraperiférica p-Pb [65].

Os autores daquele trabalho mediram a produção do méson $\mathrm{J} / \psi$ e, utilizando o fluxo de fótons virtuais, extraíram a seção de choque no próton relacionando a rapidez do méson com a energia do centro de massa. O procedimento é semelhante ao inverso daquele descrito na subseção 2.1.3. De acordo com [65], fica clara a dominância do próton na produção do méson $\mathrm{J} / \psi$ em colisões $\mathrm{pPb}$ visto que o fluxo de fótons virtuais gerado pelo $\mathrm{Pb}$ é muito mais intenso do que aquele gerado pelo próton. No que diz respeito a este trabalho, a seção de choque introduzida na fotoabsorção é compatível com o cálculo de colisões ultra-periféricas. 


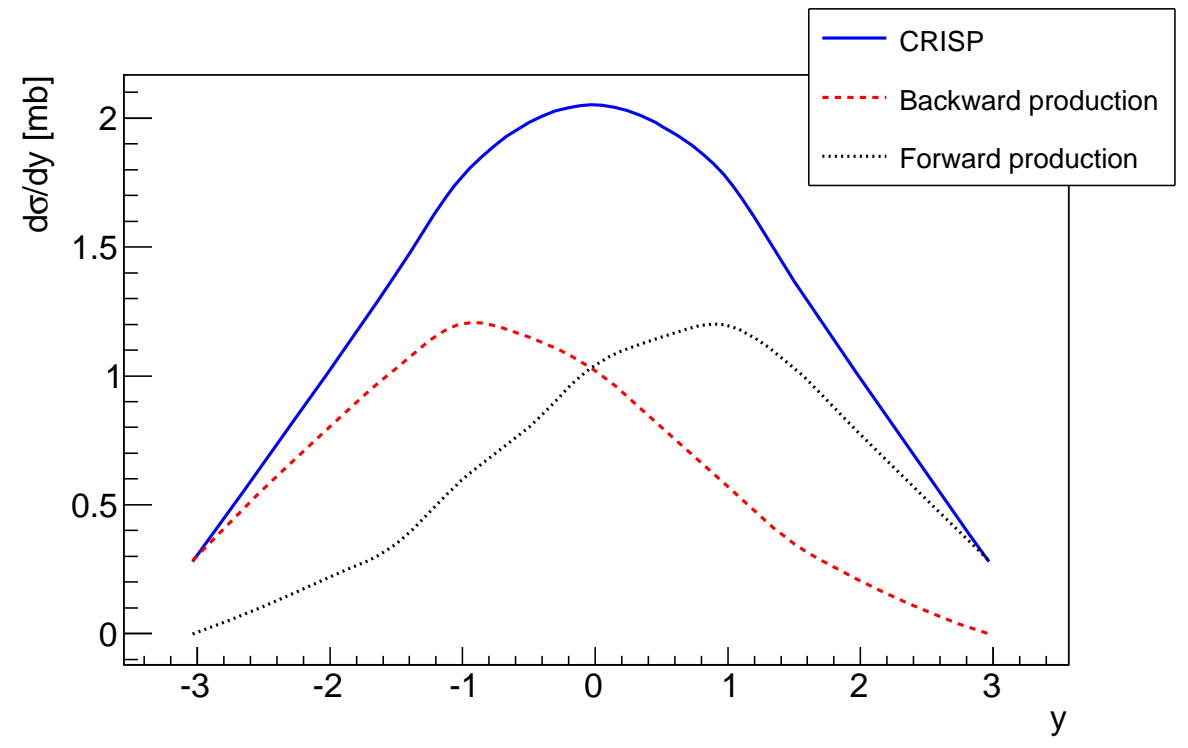

Figura 4.7: Seção de choque incoerente de fotoprodução do méson $\mathrm{J} / \psi$ em função da rapidez mostrando a contribuição de ambos os núcleos de $\mathrm{Pb}$ a $\sqrt{s_{N N}}=2.76 \mathrm{TeV}$.

Como abordado na Seção 2.4, a produção de mésons vetoriais em colisões ultra-periféricas se dá pelos processos coerente e incoerente, sendo coerente quando o fóton já no estado hadrônico interage com o núcleo alvo inteiro transferindo pouco momento, e incoerente quando a interação do fóton se dá com um nucleon do alvo, com valores um pouco maiores de momento transferido. CRISP é um modelo de cascata intranuclear e como tal recorre a uma interação inicial entre um projétil (fóton ou próton) e um nucleon do alvo seguida de interações de estado final (ou FSI na sigla em inglês), que são assim chamadas por designarem as interações dos produtos do mecanismo primário com o meio nuclear. O modelo CRISP atende assim à definição de produção incoerente.

A Figura 4.7 mostra o resultado obtido para a fotoprodução do méson $\mathrm{J} / \psi$ numa colisão $\mathrm{PbPb}$ a $\sqrt{s_{N N}}=2.76 \mathrm{TeV}$ em que se vê as contribuições devidas a cada íon.

Como descrito na subseção 2.1.3, a seção de choque para esta colisão ultra-periférica foi calculada como uma convolução do fluxo de fótons virtuais com a seção de choque fotonuclear para o processo em questão. O modelo CRISP forneceu as seções de choque de produção do méson $\mathrm{J} / \psi$ em diversos valores de energia correspondentes aos valores de rapidez a serem observados para o méson conforme a relação estabelecida pela Equação (2.22), de modo que para o intervalo de rapidez $-3<y<3$ a energia do fóton no referencial do alvo assume valores no 
intervalo $219 \mathrm{GeV}<E_{\gamma}<89 \mathrm{TeV}\left(20 \mathrm{GeV}<W_{\gamma p}<409 \mathrm{GeV}\right)$. A seção de choque incoerente em UPC no modelo CRISP corresponde finalmente à Equação (2.23). Salienta-se que o cálculo fornece apenas metade do resultado, neste caso aquele em vermelho na Figura 4.7. Dada a simetria do problema, o resultado em preto é apenas uma inversão no sinal da rapidez.

Nota-se de imediato que a produção do méson J/ $\psi$ em colisões ultra-periféricas é dominante para os menores valores de rapidez, o que corresponde aos fótons de menor energia no intervalo. Isso é compatível com o fato do fluxo de fótons virtuais ser muito mais intenso nessa região de energia caindo rapidamente para energias maiores.

Quanto à região energética relevante a este estudo, deve-se observar que a seção de choque final inclui o efeito tanto do modelo de soft dipole Pomeron a energias menores, sintetizado na Equação (2.38) [19, 21] como da aproximação por lei de potência expressa na Equação (2.40) [25] para energias maiores. Muitos modelos seguem a abordagem exposta na subseção 2.4.1 e portanto, se valem apenas da lei de potência mesmo a energias menores. No caso da colisão $\mathrm{PbPb}$, as energias de centro de massa para o par $\gamma p$ envolvidas estão distantes do limiar de produção do méson $\mathrm{J} / \psi(\sim 4.5 \mathrm{GeV})$ como se viu na Figura 2.9. Como será visto adiante, as energias na colisão AuAu colocam a descrição da seção de choque inteiramente dentro do alcance da Equação (2.38) e mais próximo do limiar de produção. O quão sensível a isso será a seção de choque final depende da parametrização de cada modelo, que precisará encontrar um equilíbrio entre os extremos da fotoprodução.

Os dados experimentais de fotoprodução de $\mathrm{J} / \psi$ em colisões $\mathrm{PbPb}$ a $\sqrt{s_{N N}}=2.76 \mathrm{TeV}$ foram publicados pela Colaboração ALICE entre 2012 e 2013 [7, 8]. Conforme descrito na Seção 2.4, a caracterização experimental deve separar as contribuições coerente e incoerente à produção do méson, começando por um corte no momento transversal do mesmo, ao que se segue uma correção que estima o grau de contaminação entre as amostras coerente e incoerente. $\mathrm{O}$ corte de momento em vigor nos dados experimentais é $p_{T}<0.3 \mathrm{GeV} / \mathrm{c}$ para processo coerente, e incoerente acima desse limiar.

A Figura 4.8 mostra o modelo CRISP comparado com os dados experimentais assim como 


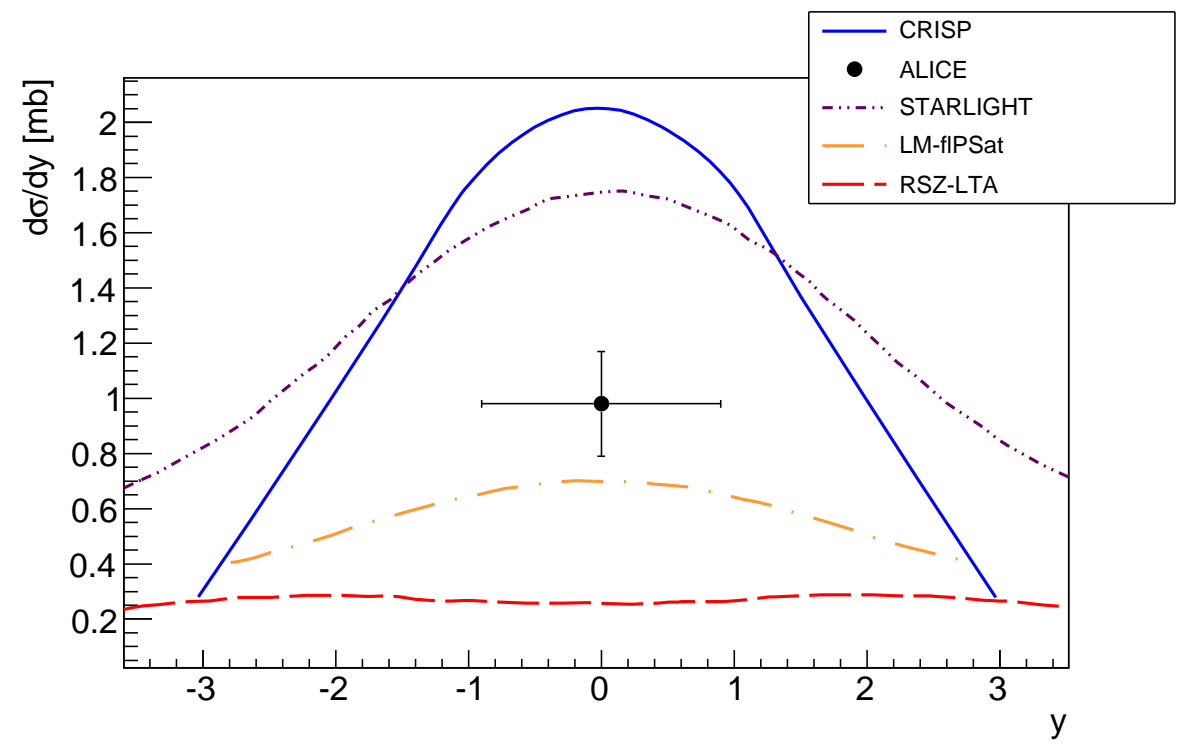

Figura 4.8: Seção de choque incoerente de fotoprodução de J/ $\psi$. Comparação com diferentes modelos.

com diferentes modelos todos obtidos da referência [8]. STARLIGHT usa o modelo de Glauber para calcular o número de nucleons participantes na colisão. A seção de choque total depende da seção de choque J/ $\psi$-nucleon e da geometria nuclear. LM-fiPsat adota um modelo de dipolo de parâmetro de impacto saturado com distribuição de glúons dentro de uma aproximação eikonal [66]. RSZ-LTA é um modelo partônico em que a seção de choque depende do quadrado da distribuição nuclear de glúons.

É possível verificar pela Figura 4.8 que o modelo CRISP superestima o valor experimental em aproximadamente 100\%. Certamente, esse não é um bom ajuste. Após levar em conta uma seção de choque $\gamma p$ consistente, um cálculo de cascata intranuclear considerado realista conforme o Capítulo 3 e as interações de estado final do méson com o meio, tamanha discrepância é um forte indício de que um modelo puramente hadrônico não baste para descrever a produção de mésons vetoriais, ao menos não nesta colisão em particular.

Todavia, vale ressaltar que os outros modelos apresentados também não chegam a ser bem sucedidos. O modelo de maior sucesso até o momento é o modelo partônico de Adeluyi e Bertulani [17], o qual já foi aplicado ao cálculo coerente [7, 8, 9]. Mesmo este modelo é compatível com diferentes distribuições de glúons. Fato é que ainda não há uma definição quanto a qual distribuição de pártons é a correta e que parametrização é a devida quando se 
recorre a Glauber.

Outra grandeza que pode ser calculada é a distribuição de momento transversal do méson, muito útil na obtenção de informações a respeito não só da interação inicial fóton-nucleon mas também das interações de estado final, especialmente do canal elástico $\mathrm{J} / \psi-N$. Embora as distribuições experimentais das contribuições coerente e incoerente não sejam acessíveis, sabemos que o modelo STARLIGHT foi aquele utilizado pela Colaboração ALICE para guiar a caracterização experimental, como explicado na Seção 2.4. Assim, comparamos a distribuição de $p_{T}$ obtida pelo modelo CRISP com dois modelos de seção de choque elástica com aquela fornecida pelo STARLIGHT na Figura 4.9.

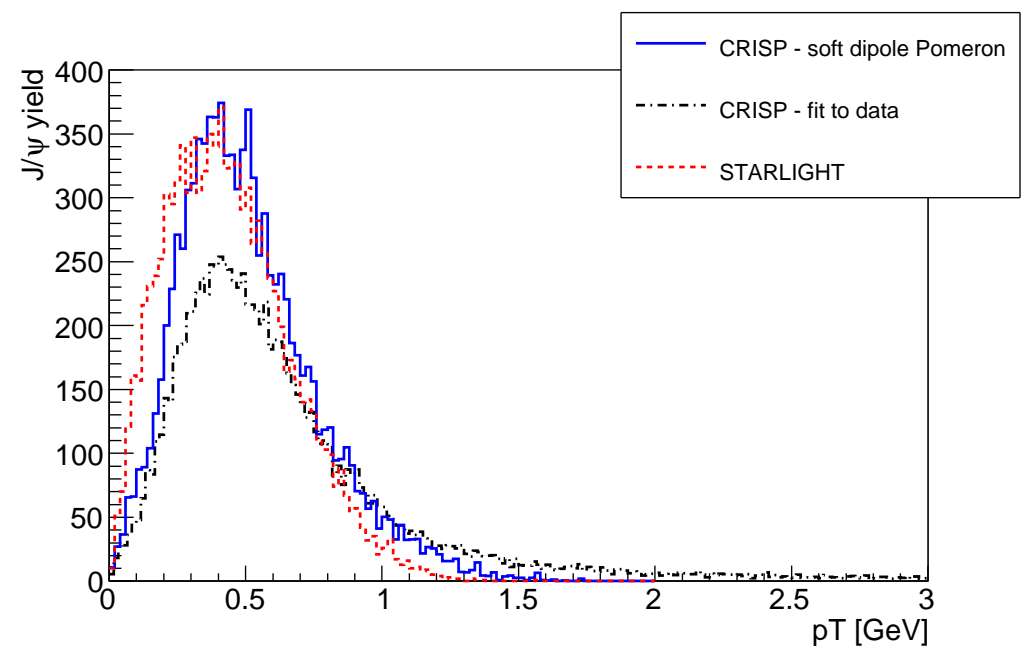

(a)

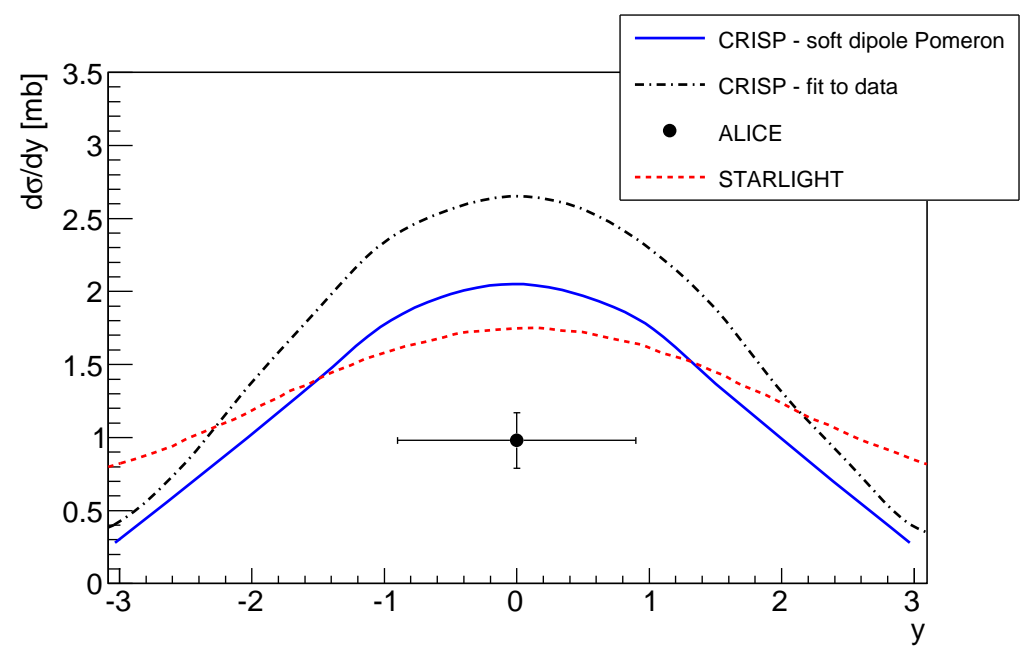

(b)

Figura 4.9: a) Distribuição de momento transversal do méson $\mathrm{J} / \psi$ obtida pelo modelo CRISP em comparação com o modelo STARLIGHT. b) Seção de choque incoerente de fotoprodução do méson $\mathrm{J} / \psi$. 
A Referência [31], que forneceu o modelo para o cálculo das interações de estado final inelásticas, menciona uma expressão que serve de alternativa ao modelo deste trabalho para o cálculo da seção de choque elástica e é dada por

$$
\left.\frac{d \sigma}{d t}\right|_{t=0}=23.15 W_{\gamma p}^{0.16}+0.034 W_{\gamma p}^{0.88}+1.49 W_{\gamma p}^{0.52}
$$

onde o primeiro termo representa a contribuição de soft Pomeron, o segundo de hard Pomeron e o terceiro é o termo de interferência. Esta expressão resulta numa distribuição de momento transversal com maior contribuição de altos momentos com se vê na Figura 4.9(a) e numa seção de choque consideravelmente mais elevada como se observa pela Figura 4.9(b).

O modelo empregado no CRISP, por outro lado, resulta numa distribuição que se estende muito menos, com prevalência de valores intermediários de $p_{T}$. Ambas as distribuições, contudo, apresentam aproximadamente o mesmo momento transversal mais provável. A seção de choque de fotoprodução, por sua vez, é consideravelmente melhor.

Uma possível explicação para essa diferença pode se encontrar no fato de que o ajuste da Equação (4.2) foi global, ou seja, levou em conta todos os dados experimentais disponíveis, como é observado na Referência [31], e se aplica a todo o intervalo de energia do centro de massa. Como já mencionado acima, o modelo CRISP segue estratégias diferentes, mais adequadas aos extremos de fotoprodução.

Nota-se ainda pela Figura 4.9 que as distribuições fornecidas pelo CRISP (soft dipole Pomeron) e pelo STARLIGHT são compatíveis exceto por dois aspectos. O primeiro deles é o pequeno deslocamento para valores mais altos de momento transversal no cálculo do modelo CRISP. O segundo, mais sutil porém perceptível, é que a distribuição calculada pelo CRISP é também mais estreita. Ambas as características são a razão imediata porque a seção de choque incoerente obtida neste trabalho é maior na região de $y=0$ (altos valores de momento transversal) e mais estreita do que aquela calculada pelo STARLIGHT.

Considerando que as diferenças são pequenas, justifica-se dizer que as distribuições de $p_{T}$ não apenas são compatíveis mas que juntas reforçam o argumento de que o mecanismo definido 
como incoerente não é suficiente para explicar os dados experimentais, devendo haver outros, em particular o coerente, marcado por baixos valores de momento transversal, como se viu na Figura 2.12.

Certas propriedades nucleares podem ser ligadas e desligadas no modelo CRISP, a saber, o movimento de Fermi e as interações de estado final do $\mathrm{J} / \psi$ com a matéria nuclear, com interesse aqui nos canais inelásticos visto serem esses os responsáveis pela supressão do méson. $\mathrm{O}$ comportamento da seção de choque em cada caso pode ser visto na Figura 4.10. A curva azul corresponde ao resultado do CRISP com todos os efeitos ligados já apresentado na Figura 4.7.

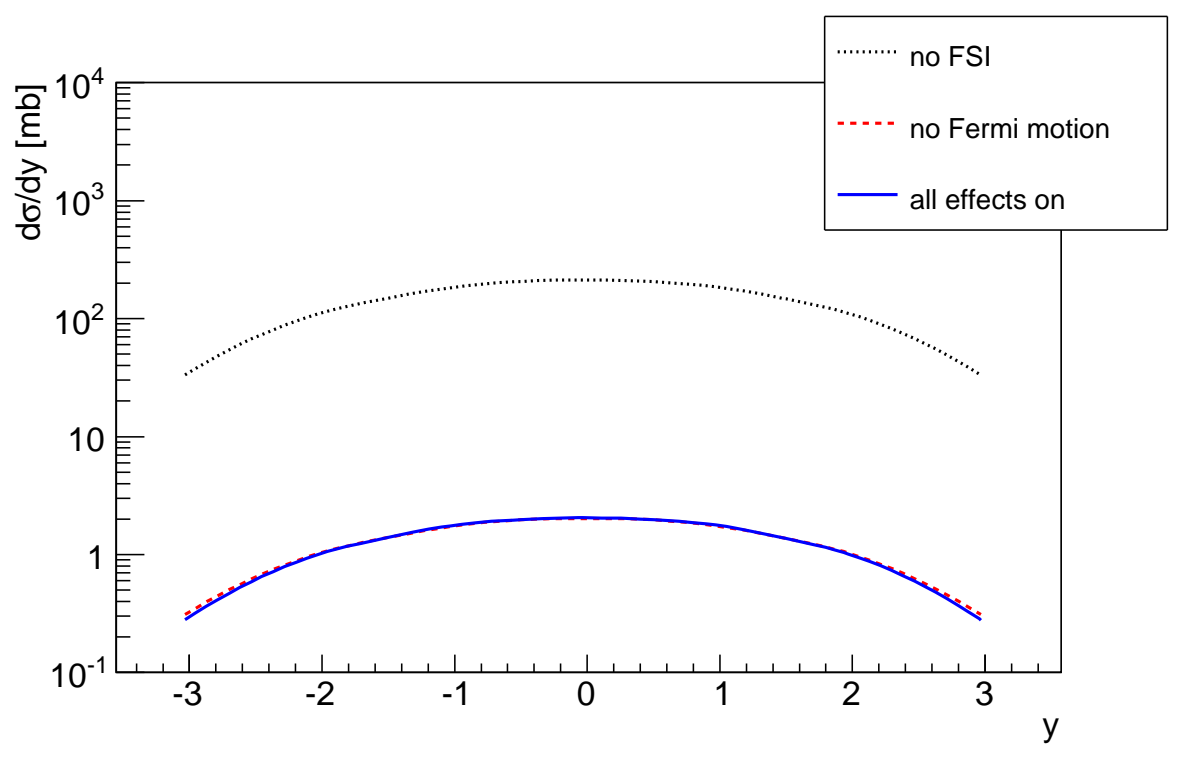

Figura 4.10: Seção de choque incoerente de fotoprodução de $\mathrm{J} / \psi$ em diferentes cenários.

Vê-se como a seção de choque é aumentada em muito com a ausência das interações de estado final o que revela a dependência dominante que o resultado possui com os canais de interação do méson com a matéria nuclear. De fato, os mésons $\mathrm{J} / \psi$ a serem emitidos do núcleo no modelo de cascata do CRISP são criados próximo à superfície do núcleo alvo. A Figura 4.11 mostra a distribuição da posição em que o J/ $\psi$ é criado conforme ele seja posteriormente emitido ou reabsorvido. A origem do sistema de coordenadas se encontra no centro do núcleo alvo e a posição registrada no gráfico é $|\vec{r}|=\sqrt{x^{2}+y^{2}+z^{2}}$.

Esta figura se soma à Figura 4.10 para mostrar o quanto as interações de estado final tem papel altamente relevante no cálculo da seção de choque. Também é possível notar pela Figura 


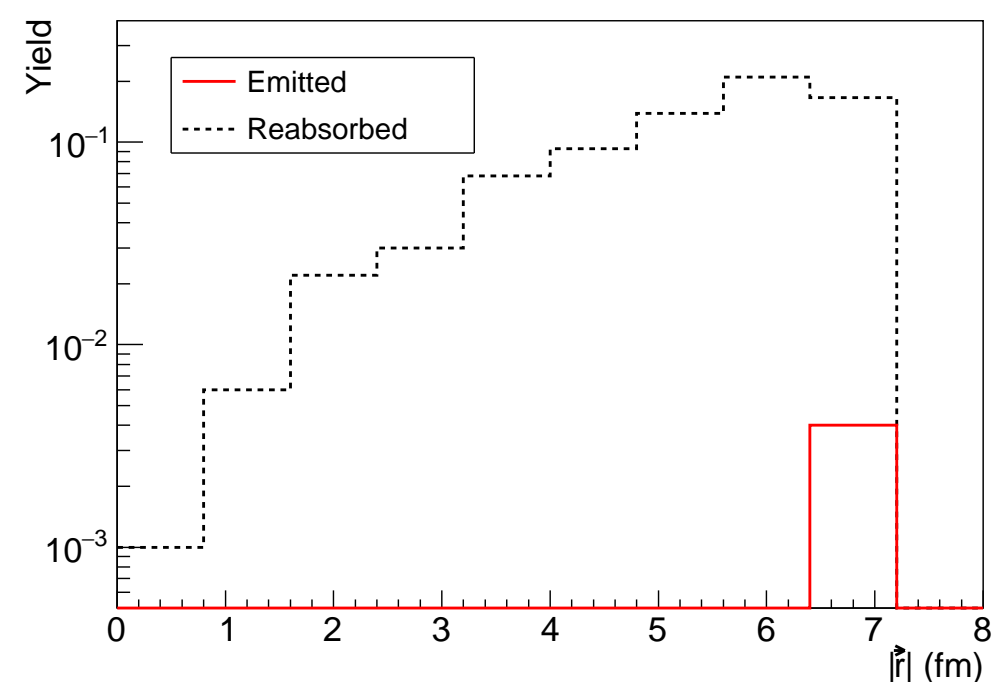

Figura 4.11: Distribuição da posição de criação do méson $\mathrm{J} / \psi$ conforme ele seja posteriormente emitido ou absorvido pelo núcleo.

4.11 que o fóton pode ser absorvido e produzir $\mathrm{J} / \psi$ em regiões mais centrais do núcleo, embora esse seja um evento menos provável. Como já mencionado no Capítulo 3, altas energias promovem o efeito de sombreamento devido à hadronização do fóton, de modo que este tende a não "enxergar" os nucleons mais centrais. Vê-se assim que dois mecanismos, sombreamento e FSI, tem forte influência sobre o resultado final da fotoprodução e ambos podem ser estudados pelo modelo de cascata do CRISP. Modelos que fazem uso do escalonamento da seção de choque, como descrito na subseção 2.4.2, embora cheguem à conclusão de que a fotoprodução do méson seja mesmo de caráter superficial, não diferenciam os efeitos do sombreamento e FSI, o que reduz o poder do método em explicar os resultados observados.

Outro aspecto das interações de estado final também foi estudado. Embora todos os canais de FSI tenham sido calculados a partir de modelos bem fundamentados, a solidez deste mecanismo dentro do modelo de cascata do CRISP foi testada com o aumento em $25 \%$ das interações de estado final. A possível necessidade deste incremento foi sugerida na Ref. [31], e seria bem vinda pois, em tese, resultaria numa menor seção de choque de fotoprodução aproximando o resultado do modelo do ponto experimental. No entanto, nenhum efeito apreciável foi observado. A explicação para isso pode ser extraída da Figura 4.11. Note-se que as J/ $\psi$ 's emitidas ocupam de fato uma faixa muito estreita na superfície nuclear, algo em torno da dimensão do nucleon. 
Vê-se ainda que na verdade muitos desses mésons foram produzidos na superfície e ainda assim foram reabsorvidos. Há uma saturação das interações de estado final. Na intensidade com que a $\mathrm{J} / \psi$ interage com a matéria nuclear, aquelas que são emitidas não apenas são produzidas na superfície mas detém um momento favorável à saída imediata do núcleo. Por isso, o aumento da FSI não produz qualquer efeito.

O movimento de Fermi foi desativado no cálculo por meio de uma recalibração da energia do centro de massa em cada instante de interação entre um fóton e um nucleon, calculando assim uma seção de choque equivalente àquela para um nucleon parado. Esse procedimento garante que a dinâmica nuclear seja preservada não causando distorções indesejadas na ocupação dos níveis de Fermi e no bloqueio de Pauli o que ocorreria caso se optasse por nucleons com momento nulo.

A influência que o movimento de Fermi tem sobre a produção sublimiar de mésons vetoriais, sem a presença do fluxo de fótons virtuais, já foi observada e analisada em trabalho anterior com o código CRISP [6]. Naturalmente, as energias consideradas neste trabalho são mais elevadas, colocando a produção de mésons acima do limiar.

Na Figura 4.10 é possível ver a produção do méson quando o movimento de Fermi se encontra inativo. O que se observa é um desvio bastante pequeno do cálculo original. Desvio esse cuja origem estatística associada ao método de Monte Carlo não pode ser descartada. Fica evidente que a fotoprodução do méson $\mathrm{J} / \psi$ é sensível a propriedades nucleares intrínsecas e um modelo hadrônico de Monte Carlo deve considerar todos os aspectos relevantes.

Além do estudo do efeito de propriedades nucleares sobre a fotoprodução, este modelo para o cálculo da seção de choque ainda pode ser testado em termos de sua estabilidade em face de diferentes parametrizações. Como o fluxo de fótons virtuais é definido a partir de constantes físicas e variáveis da colisão, resta analisar diferentes parametrizações para a probabilidade de sobrevivência, a qual depende do raio nuclear, $R_{0}$, e da densidade central, $\rho_{0}$, conforme o fator de forma nuclear da Equação (2.14). Apenas para efeito de teste de sensibilidade do modelo, aplicou-se um aumento de $7 \%$ no raio nuclear e $25 \%$ na densidade central, o que resultou numa 
probabilidade de sobrevivência como a que se vê na Figura 4.12.

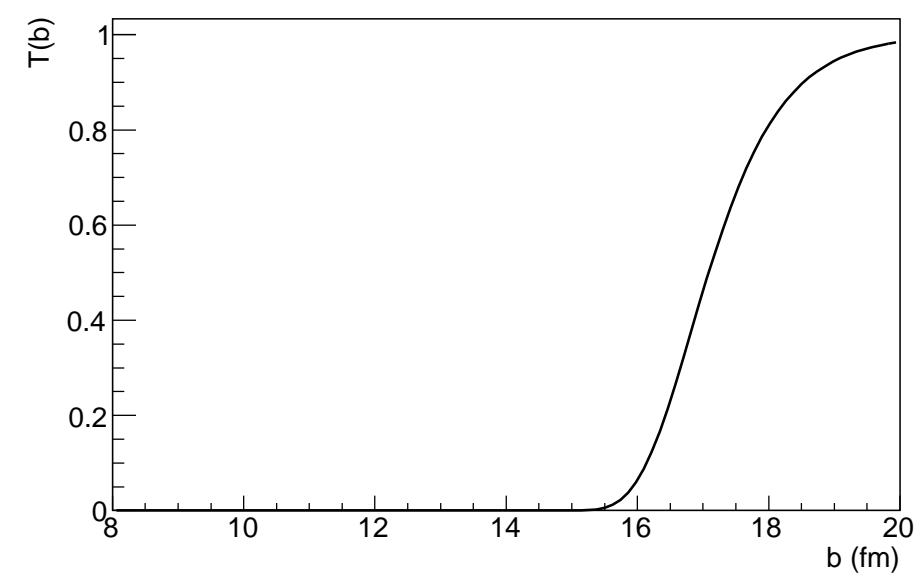

Figura 4.12: Probabilidade de sobrevivência obtida a partir de um ajuste extremo de parâmetros.

Seguiu-se o caminho de aumentar os valores dos parâmetros a fim de se observar novamente melhora no cálculo da seção de choque. Embora esta nova função probabilidade de sobrevivência difira nitidamente da forma original que se vê na Figura 2.4, a redução na seção de choque em $y=0$ foi de apenas $5 \%$. A chamada neutron skin, camada superficial de nêutrons que confere ao núcleo uma distribuição neutrônica de raio maior do que o da distribuição de carga não exibe de fato qualquer efeito na seção de choque visto que a diferença de raios no $\mathrm{Pb}$ é 0.33 fm [67] e o que se vê na Figura 4.12 é um deslocamento superior a 1 fm na probabilidade de sobrevivência com efeitos finais mínimos.

Para este trabalho também foi calculada a seção de choque de produção do méson $\mathrm{J} / \psi \mathrm{em}$ colisão AuAu a $\sqrt{s_{N N}}=200 \mathrm{GeV}$, no intervalo de rapidez $-2.5<y<2.5$, correspondendo ao intervalo $27 \mathrm{GeV}<E_{\gamma}<4 \mathrm{TeV}$ da energia do fóton ou $7 \mathrm{GeV}<W_{\gamma p}<87 \mathrm{GeV}$. O resultado pode ser visto na Figura 4.13 em comparação com o valor experimental publicado pela Colaboração PHENIX em 2009 [68]. O trabalho da Colaboração PHENIX corresponde à seção de choque total medida sem separação entre as contribuições coerente e incoerente devido a limitações estatísticas.

Estima-se, entretanto, uma maior contribuição coerente, razão porque é feita na Referência [68] comparações com previsões coerentes de alguns modelos. Duas delas, as previsões do modelo STARLIGHT e do modelo de Gonçalves e Machado são reproduzidas aqui. Também é 


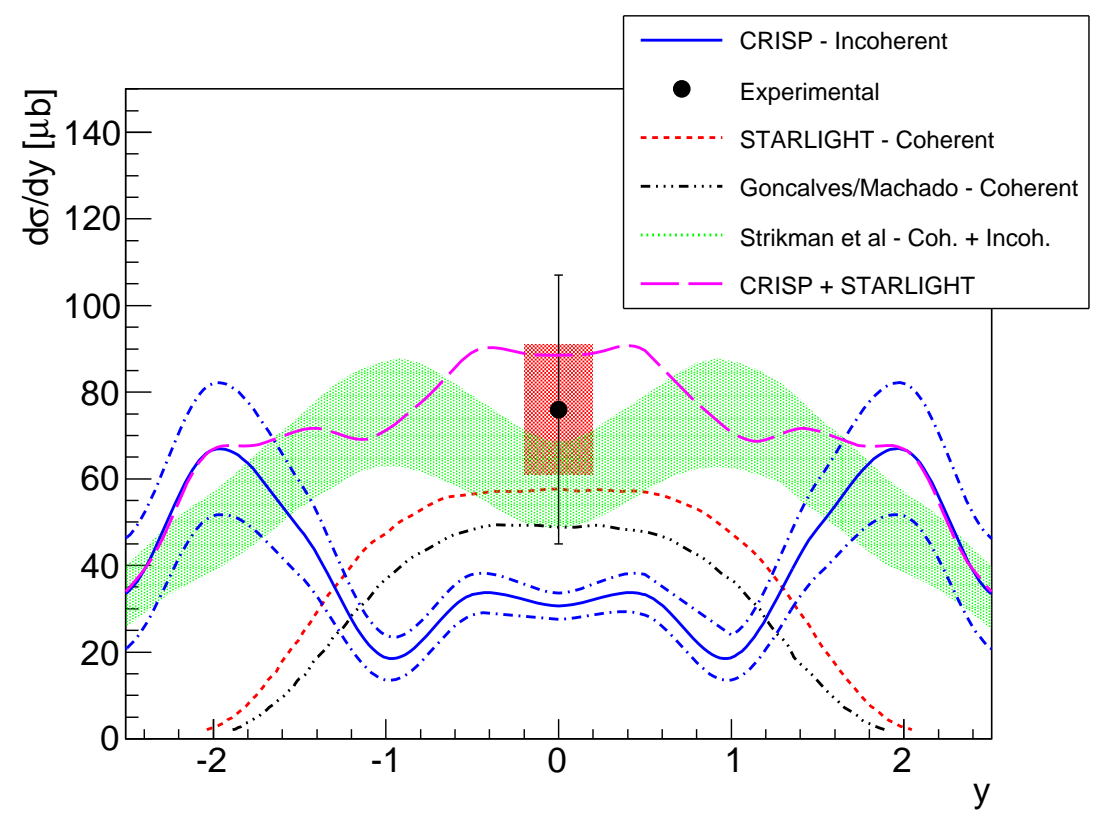

Figura 4.13: Seção de choque total de fotoprodução de $\mathrm{J} / \psi$ para colisão AuAu a $\sqrt{s_{N N}}=$ $200 \mathrm{GeV}$. Comparação com os modelos STARLIGHT, Strikman et al e Gonçalves-Machado, todos extraídos de [68] assim como o ponto experimental. As linhas (-·) delimitam a incerteza estatística no cálculo.

reproduzida aqui a previsão da seção de choque total do modelo de Strikman et al. Segundo a estimativa do experimento, a contribuição incoerente se encontra em torno de $40 \%$, ou $\sim 30 \mu b$, valor que corresponde à previsão incoerente feita pelo modelo CRISP.

A Figura 4.13 também mostra a soma das previsões dos modelos CRISP (incoerente) e STARLIGHT (coerente). Embora o comportamento geral difira daquele previsto pelo modelo de Strikman et al, tal seção de choque total resulta em bom acordo com o ponto experimental em $y=0$ e com o modelo nas demais regiões.

A ausência de mais pontos experimentais certamente reduz a extensão da análise. O resultado obtido com o código CRISP é, em todo caso, compatível com o valor experimental publicado, e vê-se que todos os modelos são bem sucedidos em descrever o resultado em $y=0$.

Neste momento, pode-se afirmar que o modelo de cascata do CRISP para a colisão $\mathrm{PbPb}$ mostrou similaridades com o cálculo segundo Glauber, o que indica que ambos os caminhos constroem fundamentalmente o mesmo cenário para a fotoprodução do méson $\mathrm{J} / \psi$. Certamente, o cálculo em uso da seção de choque de fotoabsorção e das interações de estado final do 
méson $\mathrm{J} / \psi$ com a matéria nuclear aliado a um modelo de cascata realista foram os elementos responsáveis pelo sucesso parcial alcançado.

Finalmente, vê-se que o modelo hadrônico descreve razoavelmente a fotoprodução de mésons $\mathrm{J} / \psi$ em energias mais baixas $(\leq 200 \mathrm{GeV})$ porém com limitações em energias maiores. Esse resultado parece indicar a necessidade de uma abordagem que leve em conta a estrutura nucleônica, visto que, conforme a energia da colisão e o momento transferido aumentam, interações com quarks e glúons se tornariam cada vez mais relevantes em lugar de interações com o nucleon como um todo. Dessa forma, o método da distribuição de glúons tem seu argumento reforçado por este trabalho, mas não apenas isso. Apesar do modelo CRISP não reproduzir os dados referentes à colisão $\mathrm{PbPb}$ a $\sqrt{s_{N N}}=2.76 \mathrm{TeV}$, a modelagem dos diversos processos nucleares relevantes da forma como feita aqui permite inferir com mais segurança que o modelo puramente hadrônico falha e ainda situar com maior confiança em que consiste tal falha. 


\section{Conclusões}

Foi possível neste trabalho desenvolver a metodologia de cálculo para colisões ultra periféricas, antes inexistente no modelo CRISP, com a determinação do fluxo de fótons virtuais adequado e sua convolução com a seção de choque nuclear. Avaliou-se a competência do modelo em descrever a distribuição de rapidez do méson $\mathrm{J} / \psi$ em duas colisões já realizadas experimentalmente, com destaque para a colisão $\mathrm{PbPb}$ realizada recentemente a uma energia inédita, e informações relevantes à compreensão do mecanismo de produção puderam ser obtidas através da distribuição de momento transversal do méson e do estudo do efeito de propriedades nucleares sobre a fotoprodução.

As comparações não apenas com os dados experimentais mas também com outros modelos evidenciaram o potencial do modelo de cascata do CRISP. Sua limitação em dar a correta seção de choque incoerente de produção do méson $\mathrm{J} / \psi$ fornece mais uma pista importante no caminho de determinar os aspectos físicos necessários para a devida descrição do processo. A limitação do modelo hadrônico se mostrou diretamente ligada à energia da colisão, visto que a descrição da colisão $\mathrm{AuAu}$ foi particularmente bem sucedida com piora significativa para a colisão $\mathrm{PbPb}$.

Considera-se, por isso, falar em um sucesso parcial do modelo, considerando seu estágio atual de desenvolvimento. Novos refinamentos serão buscados e o modelo será testado novamente, inclusive com a adição do estudo da produção do méson $\rho^{0}$. De fato, parte dos objetivos deste trabalho foram começar a sedimentar o caminho para estudos futuros, razão pela qual as seções de choque de fotoprodução de múltiplos píons já foram inseridas. Embora estes canais não tenham influência direta sobre a produção do méson $\mathrm{J} / \psi$, é sabido que interações $\pi N$ produzem mésons $\rho, \omega$ e $\phi$. Conhecer os diversos canais envolvidos na produção dos mésons é 
essencial à compreensão dos dados experimentais. Basta lembrar que a caracterização experimental dos dados que foram usados neste trabalho recorreram a modelos também para excluir produção de $\mathrm{J} / \psi$ por decaimento de $\psi^{\prime}$ e por interações $\gamma \gamma$.

Tendo em vista os resultados apresentados e discutidos no último capítulo e as observações acima, os objetivos estabelecidos no início deste trabalho podem-se dizer alcançados. Um projeto já foi definido para dar continuidade ao estudo do tema, tendo os pontos mencionados no último parágrafo como linhas centrais. 


\section{Referências Bibliográficas}

[1] NASSERIPOUR, R. et al. Coherent photoproduction of $\pi^{+}$from ${ }^{3} \mathrm{He}$. Phys. Rev. C, American Physical Society, v. 83, p. 034001, Mar 2011. Disponível em: $<$ http://link.aps.org/doi/10.1103/PhysRevC.83.034001>.

[2] FROMMHOLD, T. et al. Total photofission cross section for ${ }^{238} \mathrm{U}$ as a substitute for the photon absorption cross section in the energy range of the first baryon resonances. Physics Letters B, v. 295, n. 1-2, p. 28-31, 1992. ISSN 0370-2693. Disponível em: $<$ http://www.sciencedirect.com/science/article/pii/037026939290084H >.

[3] CETINA, C. et al. Photofission of heavy nuclei from 0.2 to $3.8 \mathrm{GeV}$. Phys. Rev. C, American Physical Society, v. 65, p. 044622, Apr 2002. Disponível em: $<$ http://link.aps.org/doi/10.1103/PhysRevC.65.044622>.

[4] BERTULANI, C.; NATHAN, A. Excitation and photon decay of giant resonances from high-energy collisions of heavy ions. Nuclear Physics A, v. 554, n. 1, p. 158 - 172, 1993. ISSN 0375-9474. Disponível em: $<$ http://www.sciencedirect.com/science/article/pii/0375947493903633>.

[5] BERTULANI, C. A.; KLEIN, S. R.; NYSTRAND, J. Physics of ultra-peripheral nuclear collisions. Annual Review of Nuclear and Particle Science, v. 55, n. 1, p. 271-310, 2005. Disponível em: $<$ http://www.annualreviews.org/doi/abs/10.1146/annurev.nucl.55.090704.151526>.

[6] GONZÁLEZ, I.; GUZMÁN, F.; DEPPMAN, A. Nuclear photoproduction of vector mesons within a monte carlo approach. Phys. Rev. C, American Physical Society, v. 89, p. 054613, May 2014. Disponível em: <http://link.aps.org/doi/10.1103/PhysRevC.89.054613>.

[7] ABELEV, B. et al. Coherent photoproduction in ultra-peripheral $\mathrm{Pb}-\mathrm{Pb}$ collisions at $\sqrt{s_{\mathrm{NN}}}=2.76 \mathrm{TeV}$. Physics Letters B, v. 718, n. 4-5, p. $1273-1283,2013$. ISSN 0370-2693. Disponível em: <http://www.sciencedirect.com/science/article/pii/S0370269312012257>.

[8] ABBAS, E. et al. Charmonium and $e^{+} e^{-}$pair photoproduction at mid-rapidity in ultra-peripheral $\mathrm{Pb}-\mathrm{Pb}$ collisions at $\sqrt{s_{\mathrm{NN}}}=2.76 \mathrm{TeV}$. The European Physical Journal $C$, Springer Berlin Heidelberg, v. 73, n. 11, 2013. ISSN 1434-6044. Disponível em: $<$ http://dx.doi.org/10.1140/epjc/s10052-013-2617-1>.

[9] CMS-PAS-HIN-12-009 report number:. Photoproduction of the coherent $J / \psi$ accompanied by the forward neutron emission in ultra-peripheral $\mathrm{PbPb}$ collisions at $2.76 \mathrm{TeV} .2014$.

[10] FERMI, E. Über die theorie des stoßes zwischen atomen und elektrisch geladenen teilchen. Zeitschrift für Physik, Springer-Verlag, v. 29, n. 1, p. 315-327, 1924. ISSN 0044-3328. Disponível em: <http://dx.doi.org/10.1007/BF03184853>. 
[11] FERMI, E. Sulla teoria dell' urto tra atomi e corpuscoli elettrici. Il Nuovo Cimento, Società Italiana di Fisica, v. 2, n. 2, p. 143-158, 1925. ISSN 0029-6341. Disponível em: $<$ http://dx.doi.org/10.1007/BF02961914>.

[12] JACKSON, J. D. Classical Electrodynamics. 3rd. ed. [S.1.]: John-Wiley \& Sons, Inc., 1999. ISBN 0-471-30932-X.

[13] PSHENICHNOV, I. Electromagnetic excitation and fragmentation of ultrarelativistic nuclei. Physics of Particles and Nuclei, MAIK Nauka/Interperiodica distributed exclusively by Springer Science+Business Media LLC., v. 42, p. 215-250, 2011. ISSN 1063-7796. 10.1134/S1063779611020067. Disponível em: $<$ http://dx.doi.org/10.1134/S1063779611020067>.

[14] BERTULANI, C.; GADE, A. Momdis: a glauber model computer code for knockout reactions. Computer Physics Communications, v. 175, n. 5, p. 372 - 380, 2006. ISSN 0010-4655. Disponível em: < http://www.sciencedirect.com/science/article/pii/S0010465506001937>.

[15] BERINGER, J. et al. The review of particle physics. Phys. Rev. D, v. 86, n. 010001, 2012. (Particle Data Group). Disponível em: <http://pdg.lbl.gov/index.html >.

[16] BERTULANI, C.; DOLCI, D. Charge exchange in relativistic heavy-ion collisions. $\mathrm{Nu}$ clear Physics A, v. 674, n. 3-4, p. 527 - 538, 2000. ISSN 0375-9474. Disponível em: $<$ http://www.sciencedirect.com/science/article/pii/S037594740000172X >.

[17] ADELUYI, A.; BERTULANI, C. A. Constraining gluon shadowing using photoproduction in ultraperipheral $\mathrm{pA}$ and AA collisions. Phys. Rev. C, American Physical Society, v. 85, p. 044904, Apr 2012. Disponível em: $<$ http://link.aps.org/doi/10.1103/PhysRevC.85.044904>.

[18] EDEN, R. J. Regge poles and elemetary particles. Rep. Prog. Phys., v. 34, p. 995-1053, 1971. Disponível em: <http://iopscience.iop.org/0034-4885/34/3/304>.

[19] MARTYNOV, E.; PREDAZZI, E.; PROKUDIN, A. Photoproduction of vector mesons in the soft dipole pomeron model. Phys. Rev. D, American Physical Society, v. 67, p. 074023, Apr 2003. Disponível em: <http://link.aps.org/doi/10.1103/PhysRevD.67.074023>.

[20] MEDINA, I. G. Estudo de Processos a Energias Médias e Altas pelo Método de Monte Carlo. Tese (Doutorado) — Instituto de Física da Universidade de São Paulo, 2014.

[21] MARTYNOV, E.; PREDAZZI, E.; PROKUDIN, A. A universal regge pole model for all vector meson exclusive photoproduction by real and virtual photons. The European Physical Journal C - Particles and Fields, Springer-Verlag, v. 26, n. 2, p. 271-284, 2002. ISSN 14346044. Disponível em: <http://dx.doi.org/10.1140/epjc/s2002-01058-5>.

[22] SAKURAI, J. Theory of strong interactions. Annals of Physics, v. 11, n. 1, p. $1-48$, 1960. ISSN 0003-4916. Disponível em: $<$ http://www.sciencedirect.com/science/article/pii/0003491660901263>.

[23] GELL-MANN, M.; ZACHARIASEN, F. Form factors and vector mesons. Phys. Rev., American Physical Society, v. 124, p. 953-964, Nov 1961. Disponível em: $<$ http://link.aps.org/doi/10.1103/PhysRev.124.953>. 
[24] AL., S. Chekanov et. Exclusive photoproduction of $\mathrm{j} / \psi$ mesons at hera. The European Physical Journal C - Particles and Fields, Springer-Verlag, v. 24, n. 3, p. 345-360, 2002. ISSN 1434-6044. Disponível em: <http://dx.doi.org/10.1007/s10052-002-0953-7>.

[25] CRITTENDEN, J. A. Exclusive production of neutral vector mesons at the electron-proton collider HERA. 1997. Disponível em: <arXiv:hep-ex/9704009v2>.

[26] LYKASOV, G. et al. $\omega$ n final state interactions and $\omega$-meson production from heavy-ion collisions. The European Physical Journal A - Hadrons and Nuclei, Springer-Verlag, v. 6, n. 1, p. 71-81, 1999. ISSN 1434-6001. Disponível em: $<$ http://dx.doi.org/10.1007/s100500050319>.

[27] GELL-MANN, M.; SHARP, D.; WAGNER, W. G. Decay rates of neutral mesons. Phys. Rev. Lett., American Physical Society, v. 8, p. 261-262, Mar 1962. Disponível em: $<$ http://link.aps.org/doi/10.1103/PhysRevLett.8.261>.

[28] SIBIRTSEV, A. et al. Reanalysis of antiproton production in protonnucleus and nucleus-nucleus reactions at subthreshold energies. Nuclear Physics A, v. 632, n. 1, p. 131-152, 1998. ISSN 0375-9474. Disponível em: $<$ http://www.sciencedirect.com/science/article/pii/S0375947497008099>.

[29] SWART, J. J. de; RENTMEESTER, M. C. M.; TIMMERMANS, R. G. E. PiN Newsletter, v. 13, p. 96, 1997.

[30] ROOT: An object-oriented data analysis framework - user's guide 5.26. [S.1.], December 2009. Disponível em: <http://root.cern.ch/drupal/>.

[31] SIBIRTSEV, A.; TSUSHIMA, K.; THOMAS, A. W. Charmonium absorption by nucleons. Phys. Rev. C, American Physical Society, v. 63, p. 044906, Mar 2001. Disponível em: $<$ http://link.aps.org/doi/10.1103/PhysRevC.63.044906>.

[32] ABREU, M. et al. Evidence for deconfinement of quarks and gluons from the $\mathrm{j} / \psi$ suppression pattern measured in $\mathrm{Pb}-\mathrm{Pb}$ collisions at the cern-sps. Physics Letters $B$, v. 477, n. 1-3, p. 28 - 36, 2000. ISSN 0370-2693. Disponível em: $<$ http://www.sciencedirect.com/science/article/pii/S0370269300002379>.

[33] SIBIRTSEV, A.; TSUSHIMA, K.; THOMAS, A. On studying charm in nuclei through antiproton annihilation. The European Physical Journal A - Hadrons and $\mathrm{Nu}$ clei, Springer-Verlag, v. 6, n. 3, p. 351-359, 1999. ISSN 1434-6001. Disponível em: $<$ http://dx.doi.org/10.1007/s100500050353>.

[34] SIBIRTSEV, A.; CASSING, W.; MOSEL, U. Heavy meson production in protonnucleus reactions with empirical spectral functions. Zeitschrift für Physik A Hadrons and Nuclei, Springer Berlin / Heidelberg, v. 358, p. 357-367, 1997. ISSN 0939-7922. 10.1007/s002180050339. Disponível em: <http://dx.doi.org/10.1007/s002180050339>.

[35] STARLIGHT model. [S.1.]. Disponível em: <http://starlight.hepforge.org/>.

[36] KLEIN, S. R.; NYSTRAND, J. Exclusive vector meson production in relativistic heavy ion collisions. Phys. Rev. C, American Physical Society, v. 60, p. 014903, Jun 1999. Disponível em: <http://link.aps.org/doi/10.1103/PhysRevC.60.014903>. 
[37] BALTZ, A. et al. The physics of ultraperipheral collisions at the $\{$ LHC $\}$. Physics Reports, v. 458, n. 1-3, p. 1 - 171, 2008. ISSN 0370-1573. Disponível em: $<$ http://www.sciencedirect.com/science/article/pii/S0370157307004462>.

[38] ILJINOV, A. et al. Extension of the intranuclear cascade model for photonuclear reactions at energies up to 10 gev. Nuclear Physics A, v. 616, n. 3-4, p. 575 - 605, 1997. ISSN 0375-9474. Disponível em: $<$ http://www.sciencedirect.com/science/article/pii/S0375947496004782>.

[39] ALEKHIN, S. et al. COMPILATION OF CROSS-SECTIONS. 4. gamma, neutrino, LAMBDA, SIGMA, XI, AND K0(L) INDUCED REACTIONS. 1987.

[40] DEPPMAN, A. et al. The crisp package for intermediate- and high-energy photonuclear reactions. Journal of Physics G: Nuclear and Particle Physics, v. 30, n. 12, p. 1991, 2004. Disponível em: <http://stacks.iop.org/0954-3899/30/i=12/a=016>.

[41] CUGNON, J. Proton-nucleus interaction at high energy. Nuclear Physics $A$, v. 462, n. 4, p. 751 - 780, 1987. ISSN 0375-9474. Disponível em: $<$ http://www.sciencedirect.com/science/article/pii/0375947487905756>.

[42] GONÇALVES, M. et al. Many-body cascade calculation for photonuclear reactions. Physics Letters B, v. 406, n. 1-2, p. 1 - 6, 1997. ISSN 0370-2693. Disponível em: $<$ http://www.sciencedirect.com/science/article/pii/S037026939700662X>.

[43] PINA, S. de et al. Photonuclear k+ production calculation near threshold. Physics Letters $B$, v. 434, n. 1-2, p. $1-6$, 1998. ISSN 0370-2693. Disponível em: $<$ http://www.sciencedirect.com/science/article/pii/S0370269398007266>.

[44] DEPPMAN, A. et al. Photofission and total photoabsorption cross sections in the energy range of shadowing effects. Phys. Rev. C, American Physical Society, v. 73, p. 064607, Jun 2006. Disponível em: <http://link.aps.org/doi/10.1103/PhysRevC.73.064607>.

[45] DEPPMAN, A. et al. The mcef code for nuclear evaporation and fission calculations. Computer Physics Communications, v. 145, n. 3, p. 385-394, 2002. ISSN 0010-4655. Disponível em: <http://www.sciencedirect.com/science/article/pii/S0010465502002783>.

[46] DEPPMAN, A. et al. Photofissility of heavy nuclei at intermediate energies. Phys. Rev. C, American Physical Society, v. 66, p. 067601, Dec 2002. Disponível em: $<$ http://link.aps.org/doi/10.1103/PhysRevC.66.067601>.

[47] WEISSKOPF, V. Statistics and nuclear reactions. Phys. Rev., American Physical Society, v. 52, p. 295-303, Aug 1937. Disponível em: $<$ http://link.aps.org/doi/10.1103/PhysRev.52.295>.

[48] DEPPMAN, A. et al. Photofissility of actinide nuclei at intermediate energies. Phys. Rev. Lett., American Physical Society, v. 87, p. 182701, Oct 2001. Disponível em: $<$ http://link.aps.org/doi/10.1103/PhysRevLett.87.182701>.

[49] DOSTROVSKY, I.; RABINOWITZ, P.; BIVINS, R. Monte carlo calculations of high-energy nuclear interactions. i. systematics of nuclear evaporation. Phys. Rev., American Physical Society, v. 111, p. 1659-1676, Sep 1958. Disponível em: $<$ http://link.aps.org/doi/10.1103/PhysRev.111.1659>. 
[50] BOHR, N.; WHEELER, J. A. The mechanism of nuclear fission. Phys. Rev., American Physical Society, v. 56, p. 426-450, Sep 1939. Disponível em: $<$ http://link.aps.org/doi/10.1103/PhysRev.56.426>.

[51] V.V.; PASHKEVICH. On the asymmetric deformation of fissioning nuclei. $\mathrm{Nu}$ clear Physics A, v. 169, n. 2, p. 275-293, 1971. ISSN 0375-9474. Disponível em: $<$ http://www.sciencedirect.com/science/article/pii/0375947471908840>.

[52] WILKINS, B. D.; STEINBERG, E. P.; CHASMAN, R. R. Scission-point model of nuclear fission based on deformed-shell effects. Phys. Rev. C, American Physical Society, v. 14, p. 1832-1863, Nov 1976. Disponível em: $<$ http://link.aps.org/doi/10.1103/PhysRevC.14.1832>.

[53] BROSA, U.; GROSSMANN, S.; MÜLLER, A. Nuclear scission. Physics Reports, v. 197, n. 4, p. 167-262, 1990. ISSN 0370-1573. Disponível em: $<$ http://www.sciencedirect.com/science/article/pii/037015739090114H $>$.

[54] ANDRADE-II, E. et al. Fragment mass distributions in the fission of heavy nuclei by intermediate- and high-energy probes. Journal of Physics G: Nuclear and Particle Physics, v. 38, n. 8, p. 085104, 2011. Disponível em: <http://stacks.iop.org/0954$3899 / 38 / \mathrm{i}=8 / \mathrm{a}=085104>$.

[55] DEPPMAN, A. et al. Monte carlo calculation of fragment distributions in nuclear reactions. Science and Technology of Nuclear Installations (Print), v. 2012, p. 1, 2012.

[56] DEPPMAN, A. et al. Photofission of ${ }^{232} \mathrm{Th}$ and ${ }^{238} \mathrm{U}$ at intermediate energies. Phys. Rev. C, American Physical Society, v. 87, p. 054604, May 2013. Disponível em: $<$ http://link.aps.org/doi/10.1103/PhysRevC.87.054604>.

[57] KIKUCHI, K.; KAWAI, M. Nuclear matter and nuclear reactions. [S.1.]: North-Holland, Amsterdam, 1968.

[58] CANAL, C. A. G.; SANTANGELO, E. M.; VUCETICH, H. Nucleon effective mass and the $a$ dependence of structure functions. Phys. Rev. Lett., American Physical Society, v. 53, p. 1430-1432, Oct 1984. Disponível em: $<$ http://link.aps.org/doi/10.1103/PhysRevLett.53.1430>.

[59] LEVINGER, J. S. The high energy nuclear photoeffect. Phys. Rev., American Physical Society, v. 84, p. 43-51, Oct 1951. Disponível em: $<$ http://link.aps.org/doi/10.1103/PhysRev.84.43>.

[60] RODRIGUES, T. E. et al. Photonuclear reactions at intermediate energies investigated via the monte carlo multicollisional intranuclear cascade model. Phys. Rev. C, American Physical Society, v. 69, p. 064611, Jun 2004. Disponível em: $<$ http://link.aps.org/doi/10.1103/PhysRevC.69.064611>.

[61] JAMES, F. Monte carlo phase space. Lecture Series - CERN 68-15, 1968. (description of the unpublished Raubold-Lynch generator).

[62] GABRIEL, E. et al. Open MPI: Goals, concept, and design of a next generation MPI implementation. In: Proceedings, 11th European PVM/MPI Users' Group Meeting. Budapest, Hungary: [s.n.], 2004. p. 97-104. Disponível em: <http://www.open-mpi.org/>. 
[63] AL, M. G. et. GNU Scientific Library Reference Manual. 3th. ed. [s.n.], 2009. ISBN 0954612078. Disponível em: <http://www.gnu.org/software/gsl/>.

[64] BRAGHIERI, A. et al. Total cross section measurement for the three double pion photoproduction channels on the proton. Physics Letters $B$, v. 363, n. 1-2, p. 46 - 50, 1995. ISSN 0370-2693. Disponível em: $<$ http://www.sciencedirect.com/science/article/pii/037026939501189W >.

[65] ABELEV, B. et al. Exclusive $\mathrm{J} / \psi$ photoproduction off protons in ultraperipheral $p-\mathrm{Pb}$ collisions at $\sqrt{s_{\mathrm{NN}}}=5.02 \mathrm{TeV}$. Phys. Rev. Lett., American Physical Society, v. 113, p. 232504, Dec 2014. Disponível em: $<$ http://link.aps.org/doi/10.1103/PhysRevLett.113.232504>.

[66] LAPPI, T.; MÄNTYSAARI, H. J/ $\psi$ production in ultraperipheral $\mathrm{pb}+\mathrm{pb}$ and $p+\mathrm{Pb}$ collisions at energies available at the cern large hadron collider. Phys. Rev. $C$, American Physical Society, v. 87, p. 032201, Mar 2013. Disponível em: $<$ http://link.aps.org/doi/10.1103/PhysRevC.87.032201>.

[67] ABRAHAMYAN, S. et al. Measurement of the neutron radius of ${ }^{208} \mathrm{~Pb}$ through parity violation in electron scattering. Phys. Rev. Lett., American Physical Society, v. 108, p. 112502, Mar 2012. Disponível em: < http://link.aps.org/doi/10.1103/PhysRevLett.108.112502>.

[68] AFANASIEV, $\mathrm{S}$. et al. Photoproduction of $\mathrm{J} / \psi$ and of high mass $e^{+} e^{-}$ in ultra-peripheral $\mathrm{Au}+\mathrm{Au}$ collisions at $\sqrt{s_{\mathrm{NN}}}=200 \mathrm{GeV}$. Physics Letters $B$, v. 679, n. 4, p. 321 - 329, 2009. ISSN 0370-2693. Disponível em: $<$ http://www.sciencedirect.com/science/article/pii/S0370269309008983>. 